\title{
Review
}

\section{Plasma-liquid interactions: a review and roadmap}

\author{
P J Bruggeman ${ }^{1}$, M J Kushner², B R Locke ${ }^{3}$, J G E Gardeniers ${ }^{4}$, \\ W G Graham ${ }^{5}$, D B Graves ${ }^{6}$, R C H M Hofman-Caris ${ }^{7}$, D Maric 8 , J P Reid ${ }^{9}$, \\ E Ceriani $^{10}$, D Fernandez Rivas ${ }^{4}$, J E Foster ${ }^{11}$, S C Garrick ${ }^{1}$, Y Gorbanev ${ }^{12}$, \\ S Hamaguchi ${ }^{13}$, F Iza ${ }^{14}$, H Jablonowski ${ }^{15}$, E Klimova ${ }^{16}$, J Kolb ${ }^{15}$, F Krcma ${ }^{16}$, \\ P Lukes $^{17}$, Z Machala ${ }^{18}$, I Marinov ${ }^{19}$, D Mariotti ${ }^{20}$, S Mededovic Thagard ${ }^{21}$, \\ D Minakata ${ }^{22}$, E C Neyts ${ }^{23}$, J Pawlat ${ }^{24}$, Z Lj Petrovic ${ }^{8,25}$, R Pflieger ${ }^{26}$, \\ S Reuter ${ }^{15}$, D C Schram ${ }^{27}$, S Schröter ${ }^{28}$, M Shiraiwa ${ }^{29}$, B Tarabová ${ }^{18}$, \\ P A Tsai ${ }^{30}$, J R R Verlet ${ }^{31}$, T von Woedtke ${ }^{15}$, K R Wilson ${ }^{32}$, K Yasui $^{33}$ \\ and $\mathrm{G}$ Zvereva ${ }^{34}$
}

${ }^{1}$ Department of Mechanical Engineering, University of Minnesota, 111 Church Street SE, Minneapolis, MN 55455, USA

${ }^{2}$ Electrical Engineering and Computer Science, University of Michigan, 1301 Beal Ave, Ann Arbor, MI 48109-2122, USA

3 Department of Chemical and Biomedical Engineering, Florida State University, 2525 Pottsdamer Street, Tallahassee, FL 32309, USA

${ }^{4}$ Mesoscale Chemical Systems, MESA + , Institute for Nanotechnology, University of Twente, PO Box 217, 7500AE Enschede, The Netherlands

5 Mathematics and Physics, Queen's University Belfast, University Road, Belfast, BT7 1NN, UK

${ }^{6}$ Chemical and Biomolecular Engineering, University of California—Berkeley, 201 Gilman, Berkeley, CA 94720-1460, USA

7 KWR Watercycle Research Institute, PO Box 1072, 3430BB Nieuwegein, The Netherlands

${ }^{8}$ Institute of Physics, University of Belgrade, Pregrevica 118, 11080 Belgrade, Serbia

9 School of Chemistry, University of Bristol, Cantock's Close, Clifton, Bristol, BS8 1TS, UK

${ }^{10}$ Dipartimento di Scienze Chimiche, Università degli Studi di Padova, Via Marzolo, 135131 Padova, Italy

11 Nuclear Engineering and Radiological Sciences, University of Michigan, 2355 Bonisteel Blvd, Ann Arbor, MI 48109-2104, USA

12 Department of Chemistry, University of York, Heslington, York, YO10 5DD, UK

13 Center for Atomic and Molecular Physics, Osaka University, 2-6 Yamadaoka, Suita, Osaka 565-0871, Japan

14 School of Electronic, Electrical and Systems Engineering, Loughborough University,

Epinal Way, Loughborough Leicestershire, LE11 3TU, UK

15 Leibniz Institute for Plasma Science and Technology, INP Greifswald, Felix Hausdorff-Str. 2,

17489, Greifswald, Germany

16 Faculty of Chemistry, Brno University of Technology, Purkynova 118, 61200 Brno, Czech Republic

17 Pulse Plasma Systems Department, Institute of Plasma Physics CAS, v.v.i., Za Slovankou 1782-3,

Prague 8, 182 00, Czech Republic

18 Faculty of Mathematics, Physics and Informatics, Comenius University, Mlynska dolina, 84248 Bratislava, Slovakia

${ }^{19}$ Laboratoire de Physique des Plasmas, Ecole Polytechnique, route de Saclay, F-91128, Palaiseau,

Cedex, France

${ }^{20}$ Nanotechnology and Integrated Bioengineering Centre (NIBEC), University of Ulster, Newtownabbey BT37 0QB, UK

21 Department of Chemical and Biomolecular Engineering, Clarkson University, PO Box 5705, Potsdam, NY 13699-5705, USA

22 Department of Civil and Environmental Engineering, Michigan Technological University, 1400 Townsend Drive, Houghton, MI 49931, USA

23 Department of Chemistry, Research Group PLASMANT, University of Antwerp, Universiteitsplein1, BE-2610 Antwerp-Wilrijk, Belgium 
${ }^{24}$ Electrical Engineering and Computer Science, Lublin University of Technology,

38A Nadbystrzycka str., 20-618 Lublin, Poland

${ }^{25}$ Serbian Academy of Sciences and Arts, Belgrade, Serbia

26 Institut de Chimie Séparative de Marcoule, ICSM UMR 5257, CNRS/CEA/UM/ENSCM,

Centre de Marcoule, Batiment 426, BP 17171, F-30207 Bagnols-sur-Ceze Cedex, France

27 Department of Applied Physics, Technische Universiteit Eindhoven, PO Box 513, 5600 MB,

Eindhoven, The Netherlands

28 Department of Physics, York Plasma Institute, University of York, Heslington, York, YO10 5DD, UK

${ }^{29}$ Multiphase Chemistry Department, Max Planck Institute for Chemistry, 55128 Mainz, Germany

30 Department of Mechanical Engineering, University of Alberta, Edmonton, AB T6G 2G8, Canada

31 Department of Chemistry, Durham University, Lower Mountjoy, South Road, Durham, DH1 3LE, UK

${ }^{32}$ Chemical Sciences Division, Lawrence Berkeley National Laboratory, Berkeley, CA, 94720-8176 USA

${ }^{33}$ National Institute of Advanced Industrial Science and Technology (AIST), Moriyama ku,

Nagoya 463 8560, Japan

${ }^{34}$ State University of Civil Aviation, 38, Pilotov Str., St. Petersburg, 196210, Russia

E-mail: pbruggem@umn.edu

Received 18 January 2016, revised 2 May 2016

Accepted for publication 8 June 2016

Published 30 September 2016

\begin{abstract}
Plasma-liquid interactions represent a growing interdisciplinary area of research involving plasma science, fluid dynamics, heat and mass transfer, photolysis, multiphase chemistry and aerosol science. This review provides an assessment of the state-of-the-art of this multidisciplinary area and identifies the key research challenges. The developments in diagnostics, modeling and further extensions of cross section and reaction rate databases that are necessary to address these challenges are discussed. The review focusses on nonequilibrium plasmas.
\end{abstract}

Keywords: non-equilibrium plasma, plasma-liquid interaction, diagnostics, modeling, reaction rate data sets, multiphase chemistry, photolysis

(Some figures may appear in colour only in the online journal)

\section{Introduction}

Plasma-liquid interactions are becoming an increasingly important topic in the field of plasma science and technology. The interaction of non-equilibrium plasmas with a liquid state is important in many applications ranging from environmental remediation to material science and health care. Cavendish's famous work 'experiments on air' from 1785 might be the first report involving plasma-liquid interaction and dealt with the production of nitric acid by an electric spark in air [1]. Experiments dealing with the interaction of plasmas and liquids in the context of electrochemistry date back more than 100 years ago [2]. Up to about 30 years ago, the main focus in the field of plasmas in and in contact with liquids was on glow discharge electrolysis [3] and the study of breakdown of dielectric liquids for high-voltage switching [4]. These works were followed by a strong emphasis on environmental driven research exploiting the fact that plasmas in and in contact with liquids are rich sources of reactive species, such as ${ }^{\bullet} \mathrm{OH}, \mathrm{O}^{\bullet}$ and $\mathrm{H}_{2} \mathrm{O}_{2}$, and $\mathrm{UV}$ radiation [5]. Plasmas are, in fact, a form of advanced oxidation technology enabling the breakdown of organic and inorganic compounds in water [6]. Many studies on microsecond pulsed discharges in water have addressed these topics [7].

The field of analytical chemistry often uses plasma devices to prepare samples or as a sampling process for the analyses of solutions. These techniques are typically based on glow discharges with liquid electrodes [8], inductively coupled plasmas [9] and a variety of corona, dielectric barrier discharges and glow discharges as ionization sources for mass spectrometry [10]. The emphasis in these uses of plasmas is typically not to intentionally transfer reactivity from the plasma into the liquid for the purposes of making a more reactive liquid. The plasma community has greatly benefited from this work. The topics addressed in this manuscript build on this knowledge base produced by the analytical chemistry community. However, the focus here is on plasma-liquid interactions and particularly on the physical and chemical mechanisms leading to complex feedback between the plasma and liquid at the plasma-liquid interface resulting in reactivity in the liquid.

During the last 15 years, the focus of research on the interactions of plasmas with liquids has broadened to address a variety of application areas, including electrical switching [4], analytical chemistry [8, 10], environmental remediation 


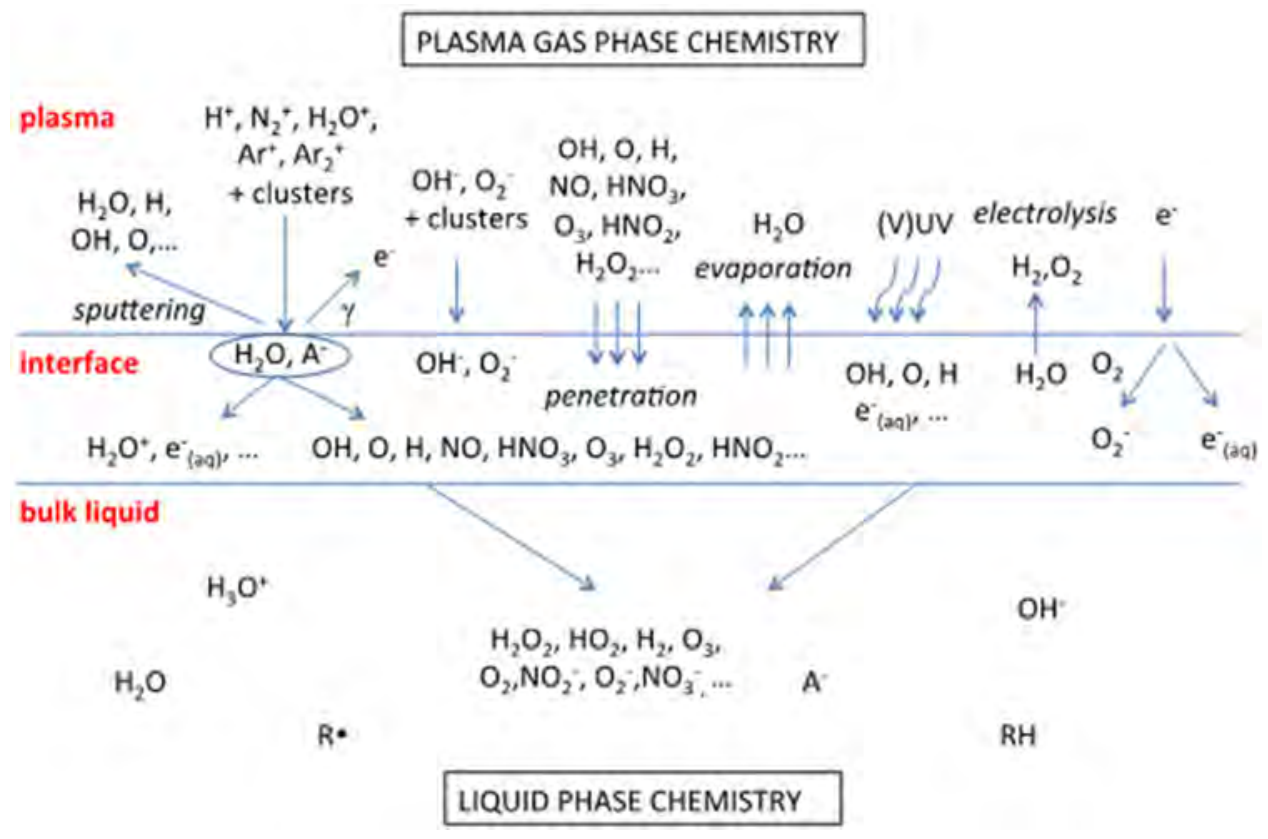

Figure 1. Schematic diagram of some of the most important species and mechanisms for an argon/humid air plasma in contact with water. Adapted with permission from [24], copyright 2014 IOP Publishing.

(water treatment and disinfection) [6], material synthesis (nanoparticles) [11], material processing (photoresist removal, plasma-polishing, polymer functionalization) $[12,13]$, chemical synthesis $\left(\mathrm{H}_{2} \mathrm{O}_{2}, \mathrm{H}_{2}\right)$ [14], sterilization and medical applications (plasma induced wound healing, tissue ablation, blood coagulation, lithotripsy) [5, 15]. These exciting opportunities have challenged the plasma community with multidisciplinary scientific questions. In addition to specialized review articles, two broader reviews focusing on the applications and the physics of plasmas in and in contact with liquids, have been published $[16,17]$.

Plasmas sustained directly in liquids are generated at one extreme by nanosecond pulsed and DC voltages [17], and at the other extreme by AC excitation of 50-60 Hz up to $\mathrm{GHz}$ microwave excitation [18]. The operating pressures range from very low values (using ionic liquids) up to very high pressure values in supercritical liquids $[19,20]$. Even at atmospheric pressure transient pressures of GPa are produced by plasma filaments generated by pulsed discharges directly in water [21]. There are many reactor geometries having different operating principles, however, these reactors can be sorted into 3 categories:

- Direct discharges in liquids,

- Discharges in the gas phase over a liquid, including when a conductive liquid is an electrode,

- Discharges in multiphase environments such as discharges in bubbles inside liquids or discharges contacting liquid sprays or foams.

Electrical breakdown and ionization in liquids have been investigated for several years. Ionization mechanisms in atomic liquids such as liquid $\mathrm{Ar}$ are relatively well understood [22]. This is less the case for complex liquids, particularly polar liquids such as water. Although it is generally thought that electrical breakdown in water occurs through formation of bubbles or in pre-existing voids, some recent results suggest that breakdown can occur without a phase change [23]. Plasmas in liquids have been investigated using imaging and optical emission spectroscopy, techniques that have enabled measuring basic plasma parameters including discharge morphology, gas temperature, electron density, and excitation temperatures. Increasing efforts have recently been devoted to modeling, but there remain many unresolved questions about the properties of plasma interaction with liquids. Two key challenges in this field were discussed in the Journal of Physics D '2012 Plasma Road Map' [24]: (a) breakdown processes and mechanisms in liquids; and (b) physical and chemical processes occurring at the plasma-liquid interface. The second challenge is particularly multi-disciplinary due to the wide range of chemical species and physical effects which involve radical and reactive species, ions, electrons, (V)UV emission, electric fields, heat and neutral gas flows across the gas-liquid interface. All these individual components are typically studied in distinct fields of research.

On August 4-8, 2014, the workshop 'Gas/Plasma-Liquid Interface: Transport, Chemistry and Fundamental Data' was held at the Lorentz Center, University of Leiden in the Netherlands. The workshop brought together scientists from different fields such as aerosol chemistry, chemical engineering, analytical chemistry, advanced oxidation technologies, microfluidics, photolysis, combustion, solvation chemistry and plasma science and technology to identify the needs and current knowledge-base of gas-liquid interface chemistry and plasma liquid-interactions. The key challenges in this field regarding transport, chemistry, the availability (or lack) of fundamental reaction rates and cross sections, and diagnostics were discussed and identified. This review can trace its origins to discussions originally held at that meeting and 

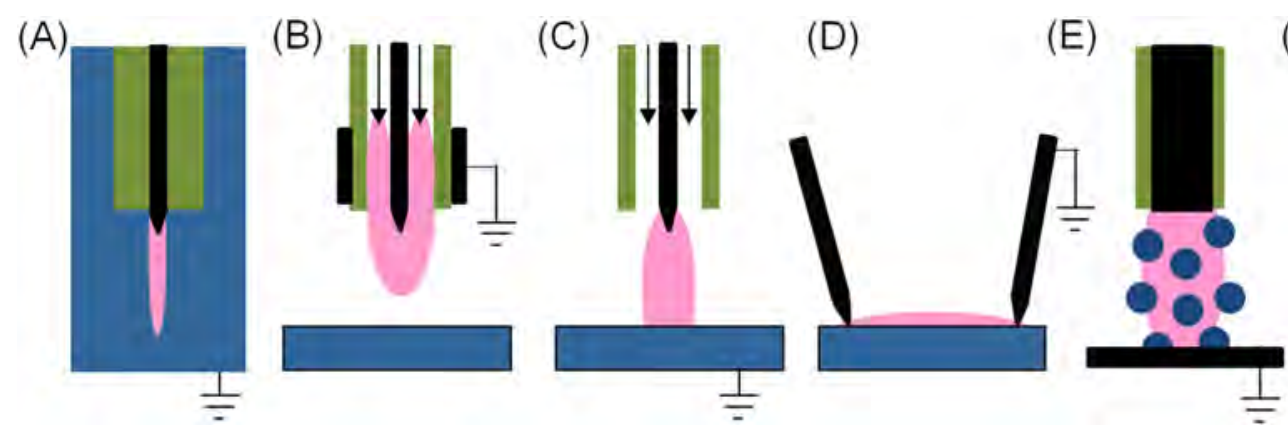

$(\mathrm{F})$

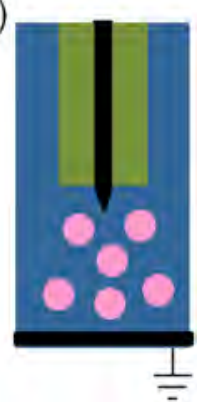

Figure 2. Schematic of different discharges used in plasma-liquid interactions: (A) direct discharge in liquid, (B)-(D) gas phase discharges and (E) and (F) multiphase discharges. In more detail: (B) plasma jet without direct contact with liquid, (C) gas phase plasma with liquid electrode, (D) surface discharge, (E) gas phase plasma with dispersed liquid phase (aerosols) and (F) discharges in bubbles. Blue $=$ liquid, pink $=$ plasma, green $=$ dielectric, black $=$ metal electrodes.

discussions continued by the authors up to the date of submission. Participants of the workshop were given the opportunity to author contributions on topics discussed at the workshop. These contributions were incorporated into sections by the first 9 authors of this manuscript. The final editing was performed by a subgroup of these 9 authors.

The goals of this review are to provide the reader with:

- An update on the state of the art in the field of nonequilibrium plasma-liquid interactions;

- An introductory description of the strongly multidisciplinary topics within the field;

- A compilation of key databases and publications;

- Identifying the key challenges in the field;

- A summary of the necessary advances in diagnostics and models required to address these key challenges;

- The critical needs of data for experimentalists and modelers;

- Guidelines on possible reference experiments, modeling and diagnostics.

The complex processes of plasmas contacting liquid water that need to be considered are shown in figure 1 for the specific example of an argon-air plasma although many other gas mixtures are important. These processes include gas phase chemistry, multiphase species transport, mass and heat transfer, interfacial reactions and liquid phase chemistry. A distinction is made between the bulk liquid and the interfacial region, the latter being the location where many important processes involving short-lived species occurs. However, different radicals will have different penetration depths according to their lifetimes, and so the thickness of the interfacial layer can vary for different processes and species.

The focus of this review is on non-equilibrium atmospheric pressure plasmas (plasmas having an average electron energy that is significant higher than the heavy particle energy). Systems in thermal equilibrium are only briefly mentioned. (The use of the term electron temperature, $T_{\mathrm{e}}$, in the text does not imply equilibrium; rather it aligns with the usual practice to equate $T_{\mathrm{e}}=(2 / 3) \varepsilon$, where $\varepsilon$ is the average energy.) This review begins with an outline of the typical conditions for initiating plasma-liquid interactions. The next sections focus on species transfer at the gas-liquid interface and photo-induced liquid phase chemistry. We then discuss heat and mass transfer processes at the plasma-liquid interface and review the literature on reaction rates and mechanisms

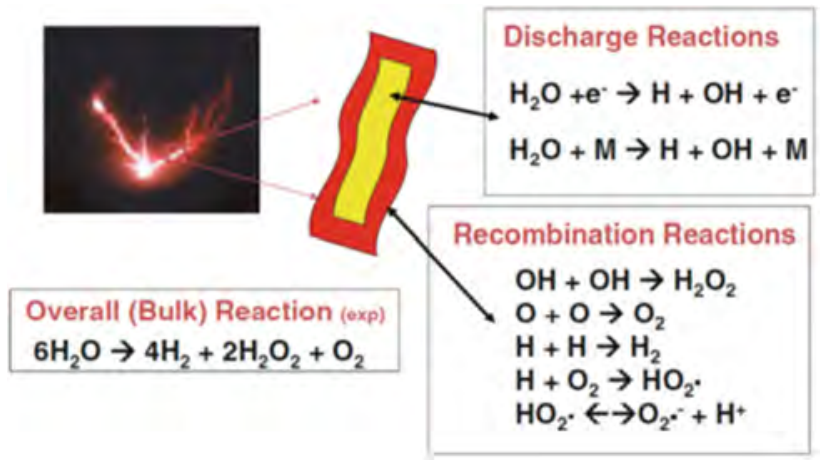

Figure 3. Schematic of the reaction chemistry in the model of Mededovic and Locke. Reproduced with permission from [38], copyright 2012 Springer.

relevant for plasma liquid interactions. Before presenting the conclusions and roadmap, the state-of-the-art and current challenges in diagnostics and modeling are discussed.

\section{Plasmas interacting with liquids: classification of conditions}

\subsection{Introduction}

Similar to gas-phase plasmas, plasma-liquid systems can be classified based on the method of generation or configurations. However, the type of interactions with liquid is of particular importance to plasma-liquid systems because it highly influences the plasma properties. One such classification scheme is (see also figure 2).

- Direct liquid phase discharges

- Gas phase plasmas producing reactivity in the liquid

- Without direct contact/electrical coupling with the liquid

- With direct contact/electrical coupling with the liquid (liquid electrode)

- At the plasma liquid interphase (surface discharges)

- Multiphase plasmas including

- Gas phase plasmas with dispersed liquid phase (aerosols)

- Gas phase plasmas dispersed in the gas phase (bubbles) in liquid 
Table 1. Typical plasma properties for four often used discharges: pulsed direct discharge in liquid, DC air glow discharge with a water electrode, pulsed plasma jet (non-touching) and filamentary dielectric barrier discharge (DBD).

\begin{tabular}{|c|c|c|c|c|}
\hline & $\begin{array}{l}\text { Direct liquid discharge } \\
{[17,21]}\end{array}$ & $\begin{array}{l}\text { DC air glow discharge } \\
{[50,52,57-59]}\end{array}$ & $\begin{array}{l}\text { Pulsed jet } \\
\text { (non-touching) } \\
{[60,61]}\end{array}$ & $\begin{array}{l}\text { Filamentary DBD } \\
\text { (single filament) } \\
{[62]}\end{array}$ \\
\hline Medium/gas & Water & Humid air & $\mathrm{He}-\mathrm{Ar}$ & Air \\
\hline Plasma generation time & $1-10 \mathrm{~s} \mu \mathrm{s}$ & Continuous & $10-100 \mathrm{~ns}$ & $1-10 \mathrm{~ns}$ \\
\hline Electron density $\left(\mathrm{m}^{-3}\right)$ & $10^{24}-10^{26}$ & $10^{18}-10^{19}$ & $10^{19}-10^{20}$ & $10^{19}-10^{21}$ \\
\hline Pressure (bar) & $10^{4}$ (peak) & 1 & 1 & 1 \\
\hline Gas temperature $(\mathrm{K})$ & $1000-7000$ & $2000-3000$ & $300-400$ & $300-400$ \\
\hline Ionization degree & $1-10^{-3}$ & $10^{-5}-10^{-7}$ & $10^{-5}-10^{-6}$ & $10^{-5}-10^{-6}$ \\
\hline Energy/power & $1 \mathrm{~J}$ per pulse & $5-100 \mathrm{~W}$ & $<10 \mu \mathrm{J}$ per pulse & $<10 \mu \mathrm{J}$ per pulse \\
\hline Power density & $\leq 10^{15} \mathrm{~W} \mathrm{~m}^{-3}$ & $\sim 10^{6} \mathrm{~W} \mathrm{~m}^{-3}$ & $<10^{12} \mathrm{~W} \mathrm{~m}^{-3}$ & $<10^{12} \mathrm{~W} \mathrm{~m}^{-3}$ \\
\hline Current & $\sim 1 \mathrm{~A}$ & $5-100 \mathrm{~mA}$ & 2-10 mA peak & $<100 \mathrm{~mA}$ \\
\hline Electron temperature $(\mathrm{eV})$ & 1 (close to LTE) & $1-2$ & $1-2$ & $2-3$ \\
\hline Electric field $\left(\mathrm{kV} \mathrm{cm}^{-1}\right)$ & $\sim 10^{3}$ & 1 (in positive column) & $1-10$ & $10-100$ \\
\hline Ion density at interface $\left(\mathrm{m}^{-3}\right)$ & $10^{24}$ & $10^{18}-10^{19}$ & $\leq 10^{16}$ & $10^{20}-10^{21}$ \\
\hline $\mathrm{UV}\left(\mathrm{m}^{-2} \mathrm{~s}^{-1}\right)$ & Broadband UV emission & $\begin{array}{l}\text { Strong UV }(\mathrm{NO}(A-X), \mathrm{OH}(A-X), \\
\left.\mathrm{N}_{2}(C-B)\right)\end{array}$ & $5 \times 10^{22}$ & $5 \times 10^{23}$ \\
\hline $\begin{array}{l}\text { Radical density } \\
\left(\text { e.g. }{ }^{\bullet} \mathrm{OH} \text { and } \mathrm{O}^{\bullet}\right) \mathrm{m}^{-3} \mathrm{~s}\end{array}$ & $\sim 10^{24}$ & $10^{21}-10^{23}$ & $10^{19}-10^{21}$ & $10^{20}-10^{21}$ \\
\hline $\begin{array}{l}\text { Reactive species flux } \\
\left(\mathrm{m}^{-2} \mathrm{~s}^{-1}\right)\end{array}$ & Extremely large gradients & $10^{23}-10^{25}$ & $5 \times 10^{21}-5 \times 10^{23}$ & $5 \times 10^{22}-5 \times 10^{23}$ \\
\hline Flow effects & Shockwaves & Thermal convection & Forced flow & Convective \\
\hline
\end{tabular}

Note: These values are estimates as a guide to typical plasma conditions.

While many other classifications can be considered, this scheme stresses the different kinds of interactions of plasmas which in turn emphasize differences in plasma generation and heat, mass and species transport. Although this review is far from exhaustive, it provides insights on the different types of plasmas in and in contact with liquids.

\subsection{Direct liquid phase plasmas}

Discharges that are generated within a liquid are highly dynamic and transient. Discharges in a liquid typically require a rapid breakdown process, which in most cases is electrically driven by strong electric fields but can also be achieved by sonoluminescent bubble implosions [25] or laser pulses [26]. Non-equilibrium discharges produced directly in the liquid by high voltage pulses are often referred to as streamer or corona discharges. These discharges are often generated by pulsed excitation in pin-to-pin or pin-to-plate configurations (see figure 2(A)). A common excitation method is discharging a capacitor by means of a short-rise-time switch such as a spark gap which produces microsecond pulsed discharges. Recently, by exploiting novel developments in the field of pulsed power there is an increased interest in nanosecond pulsed discharges in liquids [23] using pulse forming lines or solid state high voltage pulse generators.

In-liquid corona discharges are partial dischargesbreakdown, a conducting channel between the two metal electrodes, is often not achieved. The continuity of the discharge current is achieved through the liquid in the form of slow ions and displacement current instead of the mobile electrons in the plasma phase. When breakdown does occur and the reduction in impedance enables delivery of power, underwater arc discharges can be formed. Underwater arcs, which are typically thermal plasmas, have been studied in considerable detail [27]. However, their thermal nature places them outside the scope of this review.

There continues to be discussion on how discharges in liquids are generated. The details of the breakdown process depend on the excitation voltages and waveforms and the liquid properties. However, either DC or AC excitation can produce Joule heating and the formation of a vapor phase through which breakdown can occur [17]. It is generally accepted that discharges in liquids generated by microsecond voltage pulses are enabled by pre-existing bubbles or the formation of bubbles by application of the voltage [28]. However, nanosecond discharges are typically too short to form bubbles during the high voltage pulse. To date, no single comprehensive theory describes liquid electrical breakdown on nanosecond time scales although research suggests that pre-existing bubbles and field enhancement effects in the near electrode region are involved [29]. Starikovski et al [23] has observed discharge formation without measurable bubbles by using sub-ns voltage pulses of high enough $E / N$ (electric field/liquid number density) such that electron avalanche can occur in the liquid phase. The research in this area is challenged by practical difficulties such as achieving pure degassed liquids and not being able to measure (sub) micrometer gas voids due to the optical diffraction limit. Pekker and Shneider [30] have recently 
proposed that breakdown relies on generation of secondary ruptures in the vicinity of pre-existing nanopores in the liquid.

After a short time, the electron density in the avalanche of an in-liquid discharge can reach $10^{24}-10^{26} \mathrm{~m}^{-3}[7,17,21]$, and the ionization fraction can exceed $10 \%$ (depending on the pressure and temperature in the plasma filament). The gas temperatures of these in-liquid discharges can be 3000$5000 \mathrm{~K}$ [17]. The plasma channels have small diameters $\sim 10$ $\mu \mathrm{m}$ which leads to power densities in excess of $10^{15} \mathrm{Wm}^{-3}$. From measuring shock waves generated by such discharges, transient pressures are in excess of $1 \mathrm{GPa}[21,31]$. With the use of pulsed power, the electric field at the streamer head can exceed $1 \mathrm{MV} \mathrm{cm}^{-1}$ [32]. It has been proposed that these streamers propagate in water in a reduced density channel comprised of self-produced vapor or existing microbubbles $[33,34]$. These streamers can generate plasma in macroscopically sized bubbles as well [35]. In general the plasma properties are not very well characterized.

The in-liquid plasma is generated directly in the liquid or in a transient vapor phase consisting of the liquid with only minor contributions from dissolved gases, such as nitrogen and oxygen. As a result, the reactive species can be very different from those generated in typical gaseous plasmas. This is particularly the case when the liquid is a hydrocarbon, but it is also true for water. A model for plasma-inliquid, microsecond pulsed discharges was developed by Mededovic and Locke [36] and describes a plasma filament surrounded by water. A schematic of the model and reaction chemistry is shown in figure 3 . The model has two parts: an ionizing plasma which dissociates water and produces radicals, and a surrounding plasma in which the radicals recombine to produce long lived molecules. The model does not consider processes at the plasma-liquid interface at the filament edge and only electron impact dissociation and thermal dissociation of water are considered as radical production mechanisms. The calculated stable end products $\left(\mathrm{H}_{2} \mathrm{O}_{2}, \mathrm{H}_{2}\right.$ and $\left.\mathrm{O}_{2}\right)$, and densities of $\mathrm{H}^{\bullet}$ and ${ }^{\bullet} \mathrm{OH}$ up to $8.5 \times 10^{24} \mathrm{~m}^{-3}$ agree well with liquid phase measurements [36]. These values are similar to the electron densities obtained by line broadening. An overview of the typical plasma properties and conditions for pulsed direct liquid discharges is in table 1 .

Plasma parameters that directly and indirectly determine plasma processes and subsequent chemical reactions in the wake of an individual plasma channel or filament in the liquid are highly dependent on strong spatial and temporal gradients. When generated in the liquid, reactive species are able to interact on short time-scales with the medium that supports the discharge. Very different effective reaction rates or reaction efficiencies can be expected compared to gas phase discharges. For example, hydroxyl radicals $\left({ }^{\bullet} \mathrm{OH}\right)$, with reaction rates that are basically diffusion controlled will react at the plasma-liquid interface or after penetration into the liquid by only a few micrometers [37]. Understanding the formation of reactive species and secondary chemical compounds by plasmas in liquids, including reactions rates, poses considerable challenges for diagnostic methods and the development of models.

\subsection{Gas-phase plasmas}

With few exceptions that involve ionic liquids [39, 40], plasmas generated in the presence of a liquid phase are operated at atmospheric pressure or higher. These atmospheric pressure discharges have recently been reviewed in [41-45].

Gas phase electrical discharges generated between a metal pin and a water electrode have been extensively investigated [46], using a variety of discharge geometries, including the metal pin to liquid water electrode configuration [17] (see figure 2(C)). An image of a glow discharge in air with a water anode is shown in figure 4 . These discharges have similarities with contact glow discharge electrolysis in which the metal anode is submerged in the liquid and the plasma is formed in a vapor layer. The vapor layer includes gasses produced by electrolysis and evaporation, and this layer surrounds the electrode when a large current is driven through the system [2]. A review by Sen Gupta has recently addressed this topic [47]. A discharge generated between two falling film liquid electrodes has also been investigated [48].

Liquid electrodes, similar to resistive electrodes, stabilize the discharge which prevents contraction of the discharge at the electrode. The stabilization can be due to the distributed resistivity but most likely other mechanisms are also involved. Diffuse glow discharges can be generated with water electrodes even with DC excitation in atmospheric pressure air [49]. Typical operating conditions are an electrode gap of a few $\mathrm{mm}$ with currents of $5-50 \mathrm{~mA}$ and voltages of $\approx 1 \mathrm{kV}$ [50]. These discharges strongly interact with the liquid. A large fraction of the discharge power is dissipated in the liquid leading to large rates of evaporation when the liquid electrode is the cathode. Akishev et al have shown in the case of a discharge in a bubble that the evaporation is much more efficient when the liquid electrode is the cathode compared to the anode [51]. Gas temperatures in excess of $3000 \mathrm{~K}$ and electron densities of $\approx 10^{19} \mathrm{~m}^{-3}$ have been reported $[50,52]$. The electron energy distribution can be non-Maxwellian and the mean electron temperature is $\approx 1 \mathrm{eV}$ in the positive column. When the liquid electrode is the anode, self-organized patterns are often observed as shown in figure 4. Self-organization in cathode layers has been studied in considerable detail and also occurs for solid electrodes $[53,54,56]$.

Measurements of reactive species in pin-water electrode discharges are scarce, although Xiong et al [59] recently measured - $\mathrm{OH}$ densities in excess of $10^{23} \mathrm{~m}^{-3}$. Bobkova et al [57] modeled the reactive chemistry in the same discharge using a $0 D$ chemical kinetics model, and the results show a rich chemistry including large densities of ${ }^{\bullet} \mathrm{NO}, \mathrm{O}^{\bullet},{ }^{\bullet} \mathrm{OH}$ and ${ }^{\bullet} \mathrm{HO}_{2}$. The large electric field in the cathode sheath can lead to instabilities of the plasma-liquid interface $[56,58]$ which may then produce enhanced transport between the gas and liquid phase. A summary of the properties of these discharges in air is in table 1.

Pin-water electrode geometries can be used to generate corona discharges (low power) or spark discharges (high power) [49] in the gas phase. The corona discharge generates an ionic wind which leads to deformation of the liquid interface [63]. These discharges have not been studied in detail. 


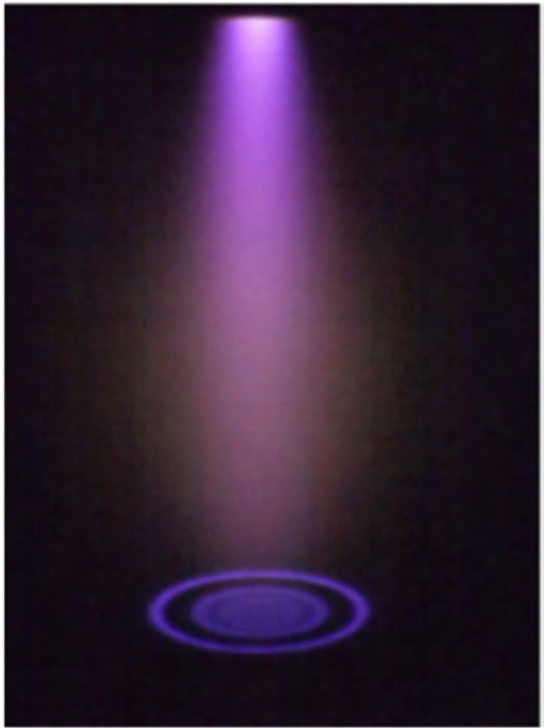

Figure 4. DC driven air glow discharge in a pin-water geometry with self-organization at the surface of the water anode electrode. Reproduced with permission from [56], copyright 2014 IOP Publishing.

Electrical discharges generated along the surface of water have also been investigated, particularly in the case of the flashover (see figure 2(D)) [64]. These devices are streamer discharges which propagate at the gas-liquid interface. Typical propagation velocities of are $1-10 \mathrm{~km} \mathrm{~s}^{-1}$ [65]. Recent studies of these devices are motivated by the potentially enhanced rate of transfer of reactive species from the gas to the liquid phase since the species are produced at the interface. Studies of surface discharges have emphasized the phenomenological aspects of their operation. More quantitative measurements by Adamovich et al have been made of (diffuse) ionization waves at the gas-liquid interface providing plasma properties and densities of reactive species [66, 67]. Surface discharges can also occur in bubbles in liquid water $[68,69]$.

A major emphasis of current research is on atmospheric pressure plasma jets (APPJs) which are often not in direct electrical contact with the liquid. The reactive species produced by APPJs are convectively transported to the liquid by the gas flow through the jet. Forced convection then enhances the transfer and generation of reactive species. Reviews on APPJs are in [45, 70]. APPJs are commonly operated as dielectric barrier discharges with either one or two outer ring electrodes [71, 72], a central needle electrode [45], or single electrode jets with capacitive coupling [73]. Excitation frequencies range from $\mathrm{DC}, \mathrm{kHz}$ and $\mathrm{MHz}$, to $\mathrm{GHz}$ in continuous wave mode or modulated formats [45, 74]. A major difference among these jets is the orientation of the electric field to the direction of flow of the gas [75]. In the cross-field jet (see figure 2(B)) the electric field is generally perpendicular to the gas flow while in the linear jet (see figure $2(\mathrm{C})$ ) the electric field is generally parallel to the gas flow. While in many cases the plasma in linear jets will be in direct contact with the liquid if the discharge is sufficiently close to the liquid, cross-field jets are often not in electrical contact with the liquid [76]. Cross-fields jets can, however, generate guided streamers

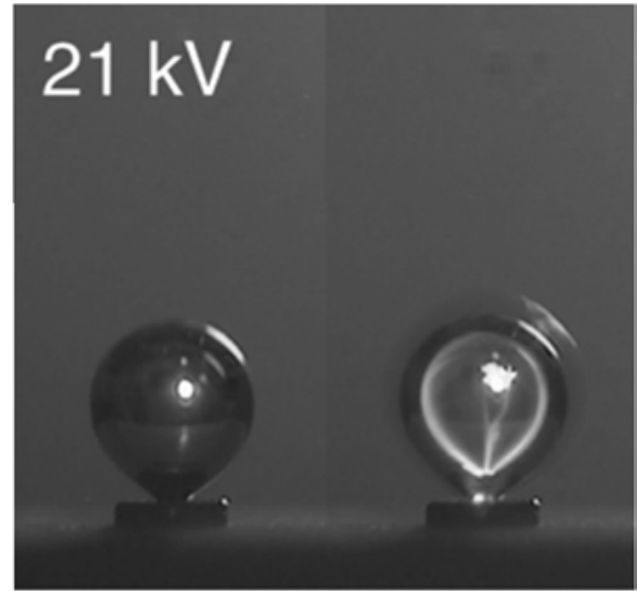

Figure 5. Filamentary surface discharge generated in a stable $\mathrm{N}_{2}$ containing bubble at an orifice. The outer diameter of the orifice is $1.6 \mathrm{~mm}$. Reproduced with permission from [69], copyright 2011 IOP Publishing.

producing electrons and ions far from the nozzle [45]. In these latter configurations, the reactive flow of species impinging on the liquid is largely devoid of ions, and the liquid interaction is dominated by neutral species produced by the plasma. Photolysis by UV photons generated by the plasma and being absorbed in the liquid may also be important. For reactive species generation in the liquid there are significant differences between the plasma touching the liquid surface and the nontouching case. Recent comparative modeling of these two cases has shown a significant influence of the touching or nottouching configurations on the reactive species formed in the liquid [61]. Initial experimental results confirm this prediction [77]. Typical plasma conditions for a nanosecond pulsed jet in a non-touching configuration are shown in table 1 . While this might not be the case for direct liquid phase discharges, ozone is commonly an important source of reactivity in gas phase discharges containing $\mathrm{O}_{2}$ or air [78, 79].

\subsection{Multiphase plasmas}

2.4.1. Gas discharges with dispersed liquid phase-aerosol plasmas. Aerosols have been extensively used in plasma technology in, for example, inductively coupled plasmas (ICPs) to identify atomic compounds in liquid samples through atomic emission spectroscopy (ICP-AES) and mass spectroscopy (ICP-MS) [80-85]. In these applications where the plasma is close to thermal equilibrium, the aerosol completely vaporizes within the plasma to produce atomized components of the aerosol $[80,84]$. Thermal plasmas with aerosols have also been widely used for surface treatment and materials deposition [86-90]. Research on these devices has not generally addressed the details of the interactions of the plasma with a 'surviving' droplet since the aerosol is often required or expected to fully vaporize within its plasma residence time. However, this experimental $[81,83,86]$ and theoretical $[85,88-90]$ research has improved our understanding of the behavior and evaporation of droplets in plasmas. Aerosol containing plasmas that are closer to being non-equilibrium have also 

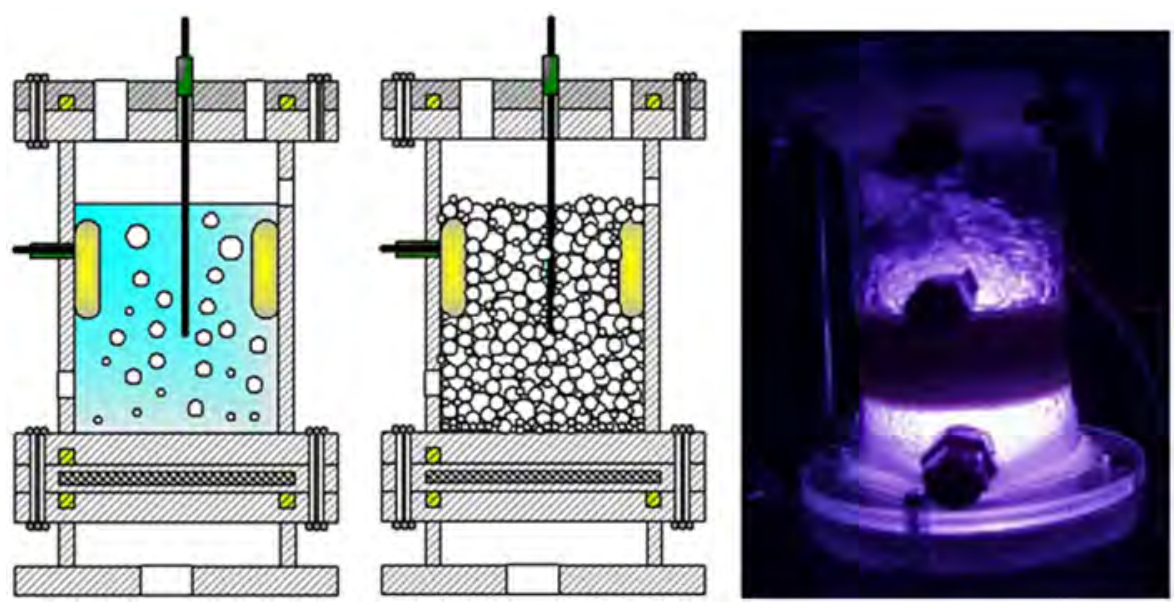

Figure 6. Schematic of a discharge with (left) bubbles and (right) foam with an image of the discharge in operation during foaming. Reproduced with permission from [118, 119], copyright 2006 Springer and copyright 2005 Acta Physica Slovaca.

been investigated. Most often the aerosol contains a precursor for plasma deposition [91-96]. Evidence of droplets surviving in non-equilibrium low-pressure [95] and atmospheric pressure [97] plasmas have been discussed and the impact of the aerosol on the plasma properties have been quantified [82, 96]. However, the impact of the plasma on the physical and chemical state of the droplets is still poorly understood [88].

Electrostatic spraying of liquids (electrospray) is a wellestablished process forming the basis of many applications (e.g. electrospinning, electrospray ionization mass spectrometry) [98-103]. There are many modes of continuous and intermittent electrospray, depending on the gas flow rate, liquid surface tension, conductivity and viscosity. Water is not a typical liquid used in electrospray due to its high surface tension resulting in the need for high voltage to produce the electrospray. This voltage is often close to the electrical breakdown of the surrounding gas. However, using water electrospray at voltages higher than the breakdown potential results in an electrical discharge in combination with the generation of a spray of water droplets [100, 104-106]. This configuration enables the efficient mass transfer of active plasma species into the water due to the micrometer dimensions of the droplet with large surface-to-volume ratios. For example, the efficient production of $\mathrm{H}_{2} \mathrm{O}_{2}$ in pulsed discharges with a water spray has been attributed to the rapid mass transfer from $\mathrm{H}_{2} \mathrm{O}_{2}$ produced in the gas phase into liquid droplets. Once in the droplets, the $\mathrm{H}_{2} \mathrm{O}_{2}$ is shielded from the highly reactive plasma environment which potentially can destroy the $\mathrm{H}_{2} \mathrm{O}_{2}$ [107].

Recent investigations have raised issues on the type of interactions between aerosol droplets and the surrounding plasma, and whether these interactions are different from those at the bulk plasma-liquid interface. An aerosol droplet experiences many processes including selective heating, charging and plasma-species diffusion that differ, at least in scale, from their analogies at the interface of a bulk liquid and a plasma. Initial results show that droplets can survive a few millisecond residence time in low-temperature atmospheric pressure plasmas and that this time is sufficient to produced many chemical reactions in the liquid aerosol [108]. Turner et al recently performed 3D simulations of the interaction of non-equilibrium atmospheric pressure plasmas with droplets containing precursors such as HMDSO for deposition purposes [109, 110].

2.4.2. Discharges in bubbles and foams. Bubbles are ubiquitous in liquid media. Energy transport at the interface during plasmas interacting with liquid water can drive instabilities leading to formation of bubbles in solution in addition to affecting the micron-sized bubbles already present. Intentional injection of bubbles into liquid water for plasma production has been extensively investigated [69, 111-113]. A common method is to use a feed gas capillary tube to inject gas into the liquid which also serves as an electrode biased with high voltage. With sufficiently high voltage, a plasma is formed inside the bubble before it breaks off of the capillary. The discharge tends to take the form of surface streamers propagating along the plasma-water interface [69], as shown in figure 5. Simulations suggest that the electron temperature in such wall hugging streamer discharges are $8-10 \mathrm{eV}$ with peak plasmas densities as high as $10^{22} \mathrm{~m}^{-3}$ [114]. Similar electron densities have been found experimentally [115]. In all cases, plasma production, and formation of reactive species largely occur in the gas phase at the plasma-liquid interface. Physical processes at the interface of bubble discharges are complex and dynamic. The electric field at the streamer head along the surface is large (several hundred $\mathrm{kV} \mathrm{cm}^{-1}$ ). Sufficiently high electric fields can locally affect chemistry through electric field-induced decomposition and charged particle acceleration [116].

Discharges in bubbles can be produced both in bubbles attached and detached from the electrode. In many respects a streamer formed at a needle electrode serves as an extension of the physical electrode when the bubble is detached [117]. Bubble dynamics inherently lead to pressure oscillations and this significantly complicates the description of discharges in bubbles. Gas temperatures and electron densities significantly vary and strongly depend on the composition of the gas in the bubble, conductivity of the liquid, discharge power, excitation voltage and size of the bubble [17]. 


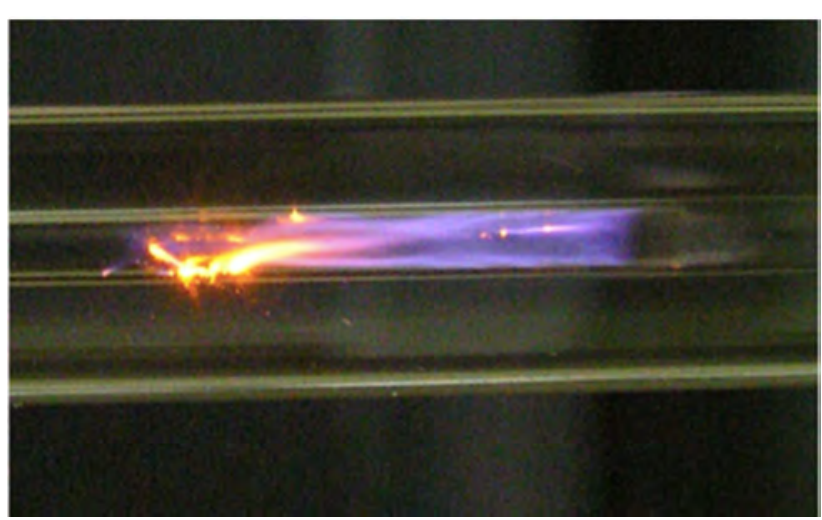

Figure 7. Image of a capillary discharge in a vapor bubble. Orange sodium emission is visible at the anode side of the bubble. The inner diameter of the capillary is $1.1 \mathrm{~mm}$. Reproduced with permission from [68], copyright 2014 IOP Publishing.

Sonoluminescence is the impulsive optical emission from plasmas produced in imploding bubbles in liquids when excited by acoustic waves without externally applied electric fields. The sonochemical plasma is formed inside the bubble as it collapses and is much smaller than the plasmas typically associated with bubbles with diameters of the plasma less than $1 \mu \mathrm{m}$. The lifetime of the plasma as measured in single-bubble experiments is $\approx 100$ ps [120-122]. In general, three regions are distinguished to account for the sonochemical reactivity

- bubble interior (hot spot at 5000-10000 K),

- bubble-liquid interface $(600 \mathrm{~K}-1900 \mathrm{~K})$ and

- bulk solution (room temperature).

Measurements of the plasma inside the bubble have been obtained through indirect methods such as optical emission spectroscopy and modeling [123]. The exact origins of the sonochemical plasma and light emission are still subject to debate [124].

An extreme situation of bubbles in water is foam. Foams are thermodynamically unstable colloidal structures that can be described as highly concentrated dispersions of gas in excess of $90 \%$ in a two-phase system with a gas as a dispersed phase and the liquid as a matrix $[125,126]$. The water is present in thin liquid film membranes [127] which separate different gas 'bubbles'. Foams can be created using whipping, gas injection, shaking and aspiration. Foam properties depend on the composition of the solution, presence of surfactants and contaminants, methods of foam formation and maintenance, temperature and pressure.

Experiments have been reported on electrical discharges and generation of active species in foaming columns [128]. The difference between a bubble and foaming reactor is schematically depicted in figure 6. The large diameter bubbles and the interfacial surface are the most preferred places for discharges occurring at low applied voltage. At higher voltages, a seemingly homogenous spatial distribution of the discharge can occur (see figure 6). $\mathrm{H}_{2} \mathrm{O}_{2}$ was produced 10 times more efficiently in a foam reactor compared to a bubbling reactor for similar plasma conditions. Foaming leads to a significant increase of mass and heat transfer (compared to bubbling) [129] and more intense plasma-liquid interactions which potentially enhance interfacial reactions. Significantly different plasma behavior was observed in investigations of positive streamers onto large bubbles and foams depending on the composition of the liquid-water versus dielectric oil [130].

2.4.3. Discharges generated in the vapor phase by local heating. A vapor phase in the liquid can be formed by intensive local heating and electrolysis by pre-discharge currents. Plasma formation can also occur in the vapor phase on metal electrodes with high DC or low frequency AC currents [3, 131, 132]. Diaphragm and capillary discharges rely on the same mechanism of plasma formation. Electrical current is constricted through a hole in a dielectric barrier separating two reservoirs filled with a conductive liquid at different electrical potentials. Applying a high voltage across the two metal electrodes inside each reservoir produces Joule heating that evaporates the liquid in the diaphragm or capillary (see figure 7). The high voltage drop now occurs in the vapor phase leading to plasma formation in the bubble. The major difference between these discharges and contact glow discharge electrolysis is that the plasma is not in contact with a metal electrode. This discharge has a long history in the development of current interrupters where the current is blocked due to formation of the vapor bubble in the opening in the dielectric [133]. The complex and dynamic nature of the vapor bubbles lead to highly dynamic and often irreproducible discharges. As a result, there are few studies describing the physics of these discharges. Most studies have focused on the generation of reactive species by capillary $[68,134]$ and diaphragm discharges $[135,136]$ in the liquid phase. Micro-discharges formed during plasma-liquid electrolytic oxidation processing have similar production mechanisms and physical properties [137].

Exploding wire discharges [138] and laser produced plasmas [26] in liquids have also been investigated. Exploding wire discharges are generated by passing a large current through a thin metal wire leading to the evaporation of the metal and a high intensity arc surrounded by liquid. A focused laser locally evaporates the liquid and produces a bubble. The high energy density can then lead to ionization. Since both approaches lead to high density, thermal plasma, these discharges are outside the scope of this review.

\section{Species transport at the gas-liquid interface}

\subsection{Introduction}

Improved insights into the physics and chemistry of transport at the plasma-liquid interface (e.g. liquid water-air) will be gained by integrating the results and methods from a wide range of related fields, including aerosol science and atmospheric chemistry [139, 140], colloidal and interfacial surface chemistry [141], evaporation and condensation [142], and phase equilibrium and gas/liquid solubility [143, 144]. 

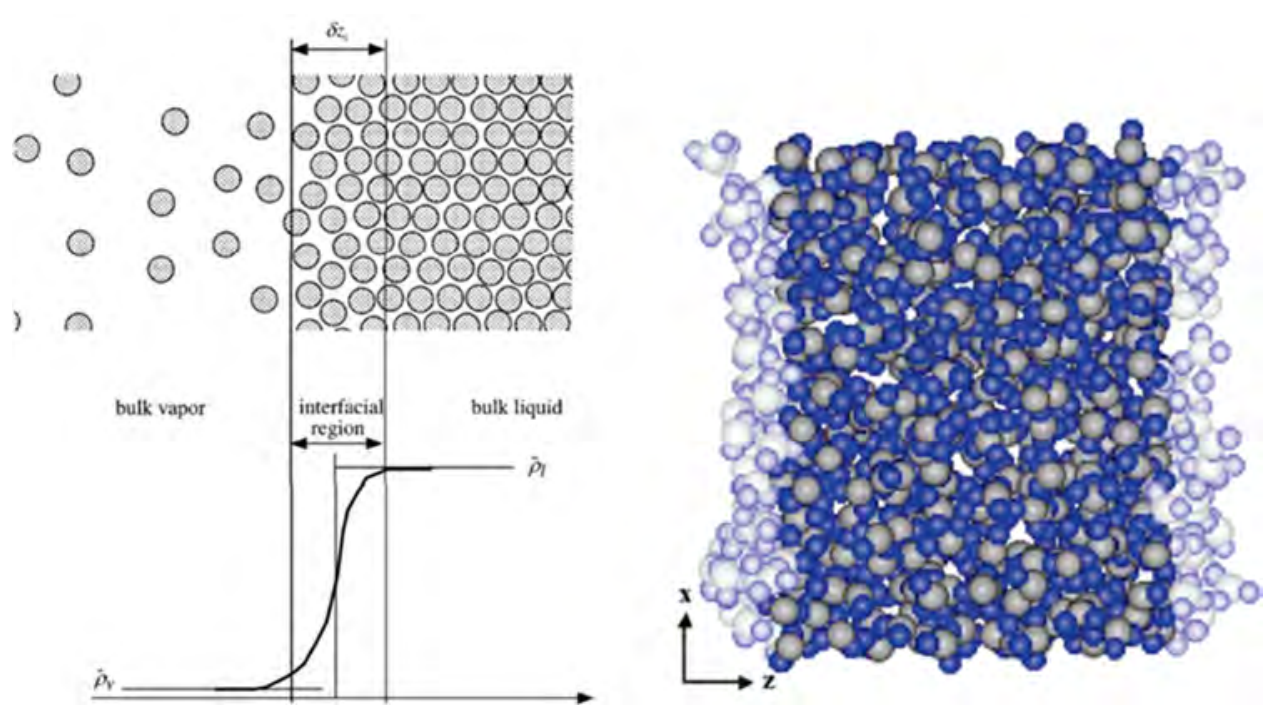

Figure 8. (left) Schematic of the gas-liquid water interface. (right) Molecular simulation of air-water interface showing two gas-water boundaries (perpendicular to the $z$-axis). The light colored water molecules indicate where the density is below $90 \%$ of the bulk liquid value. Reproduced with permission from [147, 152], copyright 2004 Springer and 2006 ACS respectively.

Analysis of the structure and dynamics of the gas-liquid interface is important for many of these fields. In this review, we will emphasize the plasma-liquid interactions where the liquid is water. Tools and methods such as classical transport theory [145], classical and non-equilibrium thermodynamics [146], and molecular methods such as Monte Carlo and molecular dynamics (MD) have been used to analyze the gasliquid interface where, for example, molecular and continuum rates of neutral species transport produce similar values [147].

In the plasma-liquid interactions considered here, chemical reactions typically occur in the bulk gas and liquid phases, and at the interface simultaneously with transport processes. Bulk gas phase and liquid phase kinetics will be described in section 6. A single reaction such as the formation of hydrogen peroxide by hydroxyl radical recombination

$$
2^{\circ} \mathrm{OH} \rightarrow \mathrm{H}_{2} \mathrm{O}_{2}
$$

can occur in the gas, the liquid, or at the interface by

$$
\begin{aligned}
2^{\circ} \mathrm{OH}_{(\mathrm{g})}+\mathrm{M} & \rightarrow \mathrm{H}_{2} \mathrm{O}_{2(\mathrm{~g})}+\mathrm{M} \\
2^{\circ} \mathrm{OH}_{(\mathrm{aq})} & \rightarrow \mathrm{H}_{2} \mathrm{O}_{2(\mathrm{aq})} \\
2^{\bullet} \mathrm{OH}_{(\text {int })} & \rightarrow \mathrm{H}_{2} \mathrm{O}_{2(\text { int })}
\end{aligned}
$$

where the interfacial reaction is followed by incorporation of

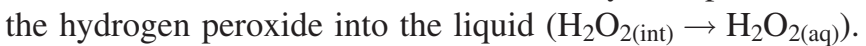
Understanding these reactions at the gas-liquid interface remains a challenge. In this section, interfacial processes, reactions and transfer mechanisms both for neutral and charged species will be reviewed.

\subsection{The gas-liquid interface}

Equilibrium analysis at phase boundaries began with Gibbs $[148,149]$ who developed the general conditions for phase equilibria in which the temperatures, pressures, and chemical

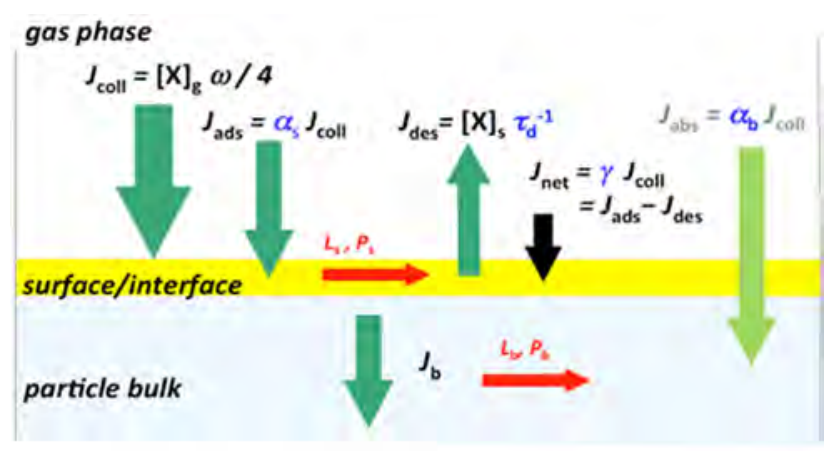

Figure 9. Schematic of key processes and fluxes in the gas uptake by atmospheric particles: gas kinetic flux of surface collisions $\left(J_{\text {coll }}\right)$, adsorption onto the particle surface $\left(J_{\text {ads }}\right)$, absorption into the particle bulk $\left(J_{\text {abs }}\right)$, desorption from the surface $\left(J_{\text {des }}\right)$, and net uptake by the condensed phase $\left(J_{\text {net }}\right)$. The red arrows indicate production and loss of chemical species by reactions at the particle surface $\left(P_{\mathrm{s}}, L_{\mathrm{s}}\right)$ or in the particle bulk $\left(P_{\mathrm{b}}, L_{\mathrm{b}}\right) .[X]_{\mathrm{g}}$ and $[X]_{\mathrm{s}}$ are the gas and surface concentrations. Reproduced with permission from [156].

potentials at a phase boundary are equal in the absence of gravitational, electromagnetic, and mechanical forces. Lewis [150] extended this view to incorporate the fugacity in place of the chemical potential, and subsequently the fugacity and activity coefficients to utilize experimentally measured mole fractions and pressure to provide a means to account for nonideal liquids and gases [151]. Simple relationships such as Henry's law for solute partitioning at the gas-liquid interface can be derived from these principles of phase equilibrium in the limits of ideal gases and ideal solutions [144]. The change in Gibbs free energy between the bulk vapor and bulk liquid in aerosols is used to define absorption while the change in free energy between the bulk liquid and interface is used to define adsorption [147].

In the field of surface science, mechanical equilibrium is used to account for surface tension and the geometrical 


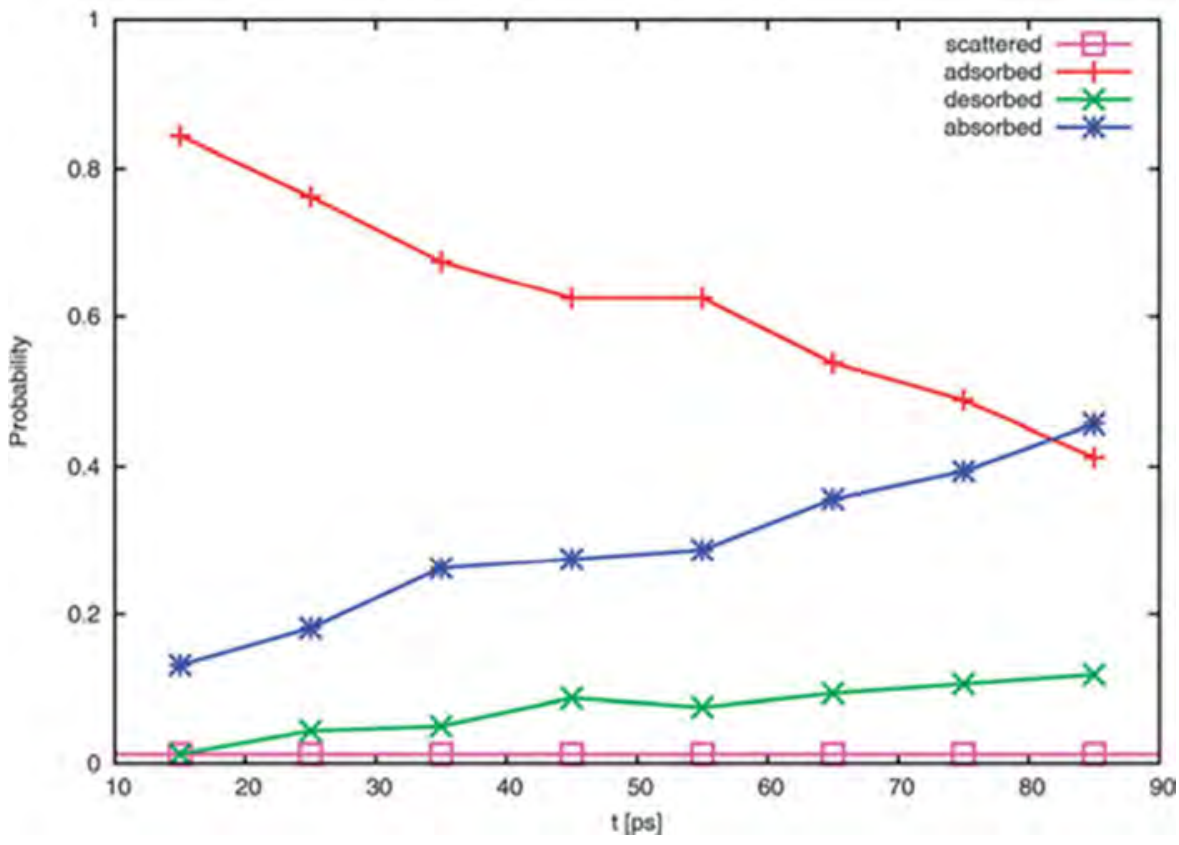

Figure 10. Scattering simulation of ${ }^{\bullet} \mathrm{NO}_{2}$ transport across gas-liquid water interface. The evolution of the probability with time for direct scattering from the surface, trapping by adsorption, desorption and absorption into the liquid. Reproduced with permission from [173], copyright 2012 Royal Society of Chemistry.

structure of the phase boundary [143]. Ultimately macroscopic properties such as surface tension and dynamic processes such as the rates of mass and energy transport at the interface are controlled by the intermolecular attractive and repulsive forces that govern the liquid and gas phases, respectively. In the liquid, the fraction of molecules having a kinetic energy sufficient to overcome the cohesive forces is small while in the gas or vapor phase the kinetic energy is higher and repulsive forces are stronger than attractive forces [142].

The transition between liquid and gas phase can be conceptualized by the change in density across the interface (see figure 8) [152] and estimates indicate that this transition occurs over a few nanometers or less [142, 147, 153, 154]. Although from a practical matter, most studies assume the interface to be atomically flat, it is likely that the interfacial structure is more complex and this complexity may be important in transport processes. For example, Jungwirth and Tobias reported that the sub-nm interface is highly dynamic, with non-stationary corrugations that may produce capillary waves [155]. Garrett found that the scale length of the transition from the vapor to liquid is $0.3-0.6 \mathrm{~nm}$ under ambient conditions, and that the rough interface has fluctuations over picoseconds with water exchanging from the bulk to the boundary over several picoseconds [147].

\subsection{Interfacial reactions and transfer mechanisms}

3.3.1. Neutral species. The interaction of a gas molecule or ion with the surface of an aerosol particle or bulk liquid involves many transport and kinetic processes [156]. A framework for describing such processes [157-159] is schematically shown in figure 9. This framework represents the net gas uptake by atmospheric particles by gas kinetic collisions,

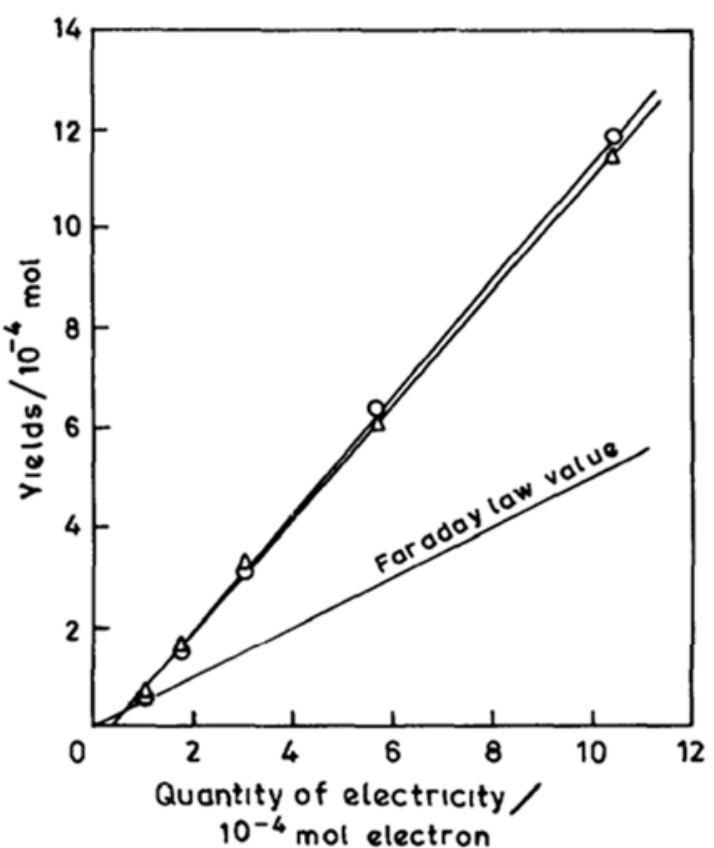

Figure 11. Variation of the yields of $\mathrm{H}_{2}(\bigcirc)$, and combined $\mathrm{H}_{2} \mathrm{O}_{2}$ (triangle) and $\mathrm{O}_{2}\left({ }^{1} \Delta\right)$ as a function of the charged transferred to the liquid during contact glow discharge electrolysis. Reproduced with permission from [238], copyright 1994 Elsevier.

adsorption onto the particle surface (aerosol), absorption into the bulk of the particle, desorption from the surface, bulk diffusion in the condensed phase, and chemical reactions at the surface of the particle or in the bulk of the particle. In some cases parameters for these processes are available in the literature $[160,161]$. Surface accommodation coefficients and desorption lifetimes can be estimated by MD simulations or density functional theory (DFT) [147, 158, 162]. Reaction rate 

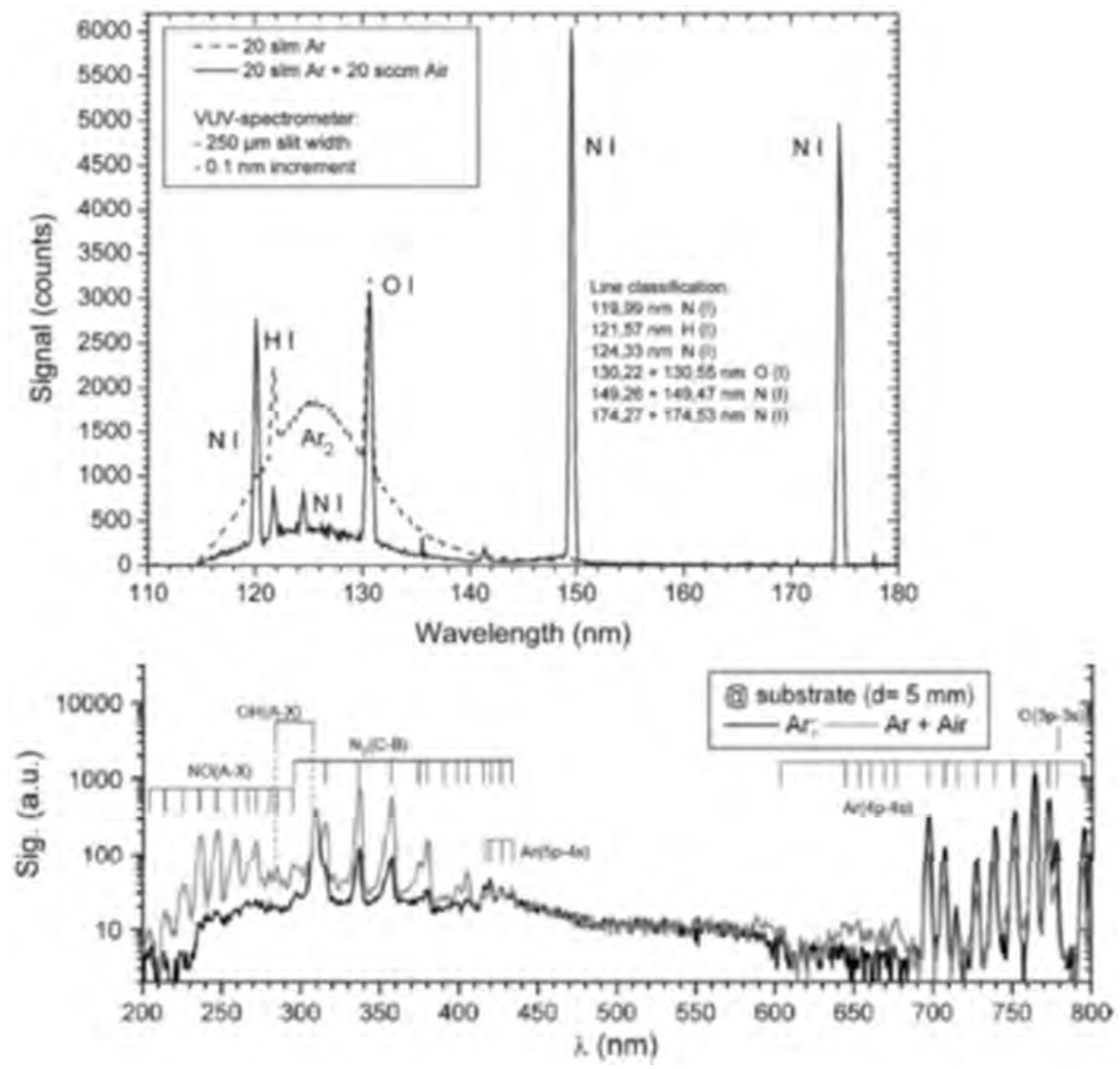

Figure 12. (top) VUV spectrum of an RF plasma jet with and without air admixture to the argon carrier gas. (bottom) UV-VIS spectra of an RF plasma jet without and with air admixture. Reproduced with permission from [257], copyright 2009 IEEE.

coefficients can also be estimated by many methods [163]. Experimental methods in aerosol science have been developed to measure surface accommodation coefficients, and include falling films devices, droplet chain systems, cloud chambers, molecular beam apparatuses, and the electrodynamic balance [164-166].

The coupling of adsorption, typically at solid surfaces, with chemical reactions is well addressed in the field of heterogeneous catalysis [167]. Two common mechanisms to describe such catalytic reactions are the Langmuir-Hinshelwood process where the overall rate is controlled by the surface reaction while the adsorption and desorption steps are at equilibrium; and the Eley-Rideal mechanism where a bulk phase species reacts directly with an adsorbed species. These mechanisms may also occur for radicals impinging on a liquid surface. The Langmuir-Hinshelwood mechanism has been applied to surfactant adsorption at gas-liquid interfaces [168, 169]. Since plasmas can generate a large flux of reactive species onto the liquid, the Eley-Rideal mechanism may be important for species adsorbed at a plasma-liquid interface.

Another approach to obtain these parameters is atomic scale modelling which provides insights to microscopic processes and phenomena at a level of detail that may be inaccessible to experiments. While certain data produced by modeling can be directly compared to macroscopic experimental measurements, such as diffusion coefficients or Henry's law constants, the advantage of atomic scale simulation is in providing microscopic data.
Examples of processes that have been analyzed include diffusion of radicals in liquid water [170], activation energy barriers [171, 172], and the structure of the solvated ${ }^{\bullet} \mathrm{OH}-$ complex $^{2}[171,172]$. A review of the molecular simulation of transport at interfaces and derivation of macroscopic properties for atmospheric aerosols is in [147]. A sample from simulations of ${ }^{\circ} \mathrm{NO}_{2}$ transport at an air-water interface is in figure 10 , showing the probability of scattering from the surface, adsorption and desorption from the interface, and absorption into the liquid [173].

Atomic scale simulation can provide the profile of free energy as a species is brought from the gas phase through the interface into the bulk of the liquid by sampling methods such as umbrella sampling $[174,175]$, the weighted histogram method [176] or umbrella integration [177] which enable extraction of the potential of mean force. The concentration ratio at two positions (e.g. in the bulk liquid and incorporated into the water-air interface) is given by

$$
\frac{c_{1}}{c_{2}}=\exp \left(-\frac{\Delta G_{12}}{R T}\right)
$$

where $\Delta G_{12}$ is the free energy difference between the species at position 1 and 2 .

Atomic scale simulations have been performed for the adsorption and absorption of small molecules and radicals in water based on classical reactive [178], nonreactive [179-181], combined quantum/classical $[182,183]$ or ab initio $[171,172]$ MD methods. While these studies have been carried out 
in the context of atmospheric chemistry, they are also relevant for plasmas contacting liquids. For hydrophobic species such as $\mathrm{O}_{2}$ and $\mathrm{O}_{3}$, the free energy difference between the bulk and gas phases is positive, a few $\mathrm{kJ} \mathrm{mol}^{-1}$, and simulations predict that their concentrations are higher in the gas phase than in the bulk liquid. Computationally derived hydration energies have good agreement with experimental values. These simulations also show a shallow minimum in the air/water interface, again a few $\mathrm{kJ} \mathrm{mol}^{-1}$, and so predict a concentration increase at the interface. From the free energy profiles, the thickness of the total interface is estimated to be $\approx 1 \mathrm{~nm}$, while the concentration increase in the topmost layer (closest to the gas phase) is over $\approx 0.5 \mathrm{~nm}$. The increases in the concentrations of $\mathrm{O}_{2}$ and $\mathrm{O}_{3}$ at the interface relative to the bulk liquid are estimated to be $\approx 40$ and 10 times, respectively. For hydrophilic species, including ${ }^{\bullet} \mathrm{OH}, \mathrm{HO}_{2}^{\bullet}$ and $\mathrm{H}_{2} \mathrm{O}_{2}$, the calculated free energy profiles indicate that these species are stabilized in the bulk of the liquid, by $19 \mathrm{~kJ} \mathrm{~mol}^{-1}, 29 \mathrm{~kJ} \mathrm{~mol}^{-1}$ and $42 \mathrm{~kJ} \mathrm{~mol}^{-1}$, respectively. They are further stabilized in the water/air interface by an additional 6,2 and $2 \mathrm{~kJ} \mathrm{~mol}^{-1}$. This leads to a concentration enhancement in the interface relative to the bulk liquid by factors of 8, 2.3 and 1.5, respectively [179]. Atomic hydrogen is however hydrophobic with a free energy of $19 \mathrm{~kJ} \mathrm{~mol}^{-1}$ [184].

The lifetime of radicals and other species at the interface should also be considered. For example, Wick et al [180] calculated a $150 \mathrm{ps}$ residence time at the interface during the uptake of ${ }^{\bullet} \mathrm{OH}$ from the gas phase to the water interface. From the radial distribution functions derived from MD simulations, it was shown that stabilization or destabilization of species at the interface and in the bulk liquid are due to differences in hydrogen bonding [182, 183, 185].

Reactive classical MD simulation, performed by Yusupov et al [178], indicate that ${ }^{\bullet} \mathrm{OH}$ is not confined to the gas-liquid interface while $\mathrm{HO}_{2}^{\circ}$ remains at the interface. This apparent contradiction with the outcomes of free energy simulations might result from the reaction-diffusion process in the liquid occurring through repeated reactions of the ${ }^{\bullet} \mathrm{OH}$ with the surrounding water molecules, exchanging an $\mathrm{H}^{\bullet}$-atom and reforming the reactants. Diffusion coefficients for ${ }^{\bullet} \mathrm{OH}$, $\mathrm{H}_{2} \mathrm{O}_{2}$ and $\mathrm{HO}_{2}^{\circ}$ were calculated to be $0.84 \AA^{2} \mathrm{ps}^{-1}(0.84 \times$ $\left.10^{-4} \mathrm{~cm}^{2} \mathrm{~s}^{-1}\right), 0.13 \AA^{2} \mathrm{ps}^{-1}\left(0.13 \times 10^{-4} \mathrm{~cm}^{2} \mathrm{~s}^{-1}\right)$ and $0.07 \AA^{2} \mathrm{ps}^{-1}\left(0.07 \times 10^{-4} \mathrm{~cm}^{2} \mathrm{~s}^{-1}\right)$, respectively. From these reactive simulations, $\mathrm{HO}_{2}^{-}$was found to react with water to form the superoxide anion, $\mathrm{O}_{2}^{\cdot-}$, and the hydronium ion, $\mathrm{H}_{3} \mathrm{O}^{+}$ reacts with another $\mathrm{HO}_{2}^{\circ}$ molecule, forming $\mathrm{H}_{2} \mathrm{O}_{2}$ and $\mathrm{O}_{2}$. $\mathrm{H}_{2} \mathrm{O}_{2}$ was only found to react when 3 water molecules are sufficiently close, forming $2 \mathrm{HO}_{2}^{\circ}+2 \mathrm{H}_{2} \mathrm{O}$.

The diffusion coefficients of solutes in gases and liquids can be estimated using standard methods [186, 187] and these values have been used in plasma-liquid models [188].

3.3.2. Ions. The transport of cations (positive ions) across the plasma-liquid interface for plasma relevant conditions is relatively unexplored [189, 190]. It is common in models to assume that the potential energy of all cations is large compared to any activation energy barrier (such as surface tension) required to enter the liquid, and so cations are mostly immediately solvated when striking the liquid. Atomic scale simulations have been performed to investigate the interaction of ions with the water surface $[191,192]$. These simulations provide insights on the sputtering mechanism of water molecules from the interface, the depth of ion penetration, and the temperature increase of the liquid induced by ion impact. It was found that even $100 \mathrm{eV} \mathrm{O}^{+}$ions do not penetrate beyond the liquid surface by more than about $3 \mathrm{~nm}$ [192]. At this energy, however, the average sputter yield was calculated to be 7.0. It was also found that not all impinging ions are incorporated into the liquid, but some ions are reflected upon impact. A local temperature increase of about $50 \mathrm{~K}$ at a $5 \mathrm{~nm}$ depth in the liquid was also observed upon a single ion impact.

However, the mean-free path of an ion at atmospheric pressure (and room temperature) is less than $1 \mu \mathrm{m}$ which is significantly smaller than the typical sheath thickness of a steady-state plasma at atmospheric pressure. The ion energy impinging on a liquid can be approximated by the electric field times the mean-free path $(E \times l)$. With an electric field of $100 \mathrm{kV} \mathrm{cm}^{-1}$ or less, ion energies in steady-state atmospheric pressure plasmas are expected to be of the order of a few $\mathrm{eV}$ at most, much lower than those considered in the modeling studies. A small fraction of very energetic ions can be produced in specific cases of transient nanosecond pulsed discharges where ionization waves strike liquids, but even in these cases the average ion energy remains of the order of at most a few eV [193].

Estimates of ion energies in glow discharges with liquid electrodes have been made from the yield of oxidized species in the liquid. The estimated values of ion energy are $90 \mathrm{eV}$ assuming that the plasma transfers its oxidative power mainly through energetic ion impact on the water cathode [194]. To achieve this ion energy would require that the sheath collapses to a thickness close to the ion mean-free path, or less than $1 \mu \mathrm{m}$. UV photons and neutral reactive species from the gas phase can also transfer oxidative power from the plasma to the liquid phase, and so perhaps less energetic ions are needed. These interactions require further investigation.

The transport of anions (negative ions) across the plasmaliquid interface is less clear. Since anions typically do not have a positive potential energy, they are more energetically equivalent to neutral species than to positive ions. However, the anions are likely to be far more polarizable than either neutrals or cations. It is not well understood how negative ions penetrate the plasma-liquid interface. The reverse process is poorly understood - for example, do cations leave the liquid?

Finally, a secondary electron emission mechanisms upon ion impact on a liquid surface has been postulated by Mezei et al [46, 194]. This is an extremely important process as it is highly likely that the plasma is sustained by processes dramatically different than the secondary electron process at the surface of metal electrodes. Direct electron emission from pure water requires $6.1 \mathrm{eV}$ and could be achieved by photoemission with wavelengths shorter than $203 \mathrm{~nm}$. The hydrated electron only requires an energy of $1.56 \mathrm{eV}$ to leave the water surface [195]. Based on these observations, Cserfalvi and Mezei proposed a secondary electron emission process involving aqueous electrons with and without the involvement of protons depending on the $\mathrm{pH}$ of the liquid [194]. Estimates of the secondary electron emission coefficient fit experimentally 
obtained data obtained as a function of $\mathrm{pH}$. Secondary electron emission based on the emission of electrons from negative ions in salt solutions has also been suggested by Gaisin and Son [196].

3.3.3. Electrons. Electron-induced reactions at the liquid interface can be generally classified into two types: (a) highly energetic electrons which could (vibrationally) excite, dissociate (including dissociative attachment) or ionize water molecules, and (b) low energetic electrons which adsorb and absorb, and eventually become solvated electrons $\left(e_{\text {(gas) }}^{-} \rightarrow e_{(\mathrm{aq})}^{-}\right)$. Electrons are hydrophobic with a large solvation energy of $-156 \mathrm{~kJ} \mathrm{~mol}^{-1}$ [184]. Although intensively studied in many contexts, the transport of electrons across the plasma-liquid interface is poorly understood [197, 198]. Many experiments have been performed on the solvation and transport of electrons within water and water clusters. The electron is often initially inserted into the water or cluster as a high energy particle - an electron beam, or generated internally by radiolysis or photoionization. These electrons have energies far in excess of any barrier that might inhibit their adsorption. However, the transport of low energy (less than $1 \mathrm{eV}$ ) electrons into the water is less clear [199, 200]. In radiation chemistry a solvated electron is formed after the electron produced by the absorption of ionizing radiation is thermalized and the surrounding molecules orient in response to the negative charge of the electron [201]. Solvated electrons in water are also called hydrated electrons.

The lifetime and the state of these solvated electrons $\left(e_{(\mathrm{aq})}^{-}\right)$ are not well understood [202-209]. For most practical applications it might be sufficient to consider that solvated electrons either recombine at the interface through an interface neutralization mechanism, with, for example, ions reaching the interface from the plasma

$$
e_{(\mathrm{aq})}^{-}+\mathrm{A}_{(\mathrm{g})}^{+} \rightarrow \mathrm{A}_{(\mathrm{g})}
$$

These reactions are analogous to those occurring on a solid surface in contact with a plasma.

There are many other reactions of solvated electrons in and with liquid water as reported in the radiation chemistry literature. The following reactions have been reported to be important for many relevant photolysis conditions in the absence of other scavengers [210]:

$$
\begin{gathered}
2 e_{(\mathrm{aq})}^{-}+2 \mathrm{H}_{2} \mathrm{O} \rightarrow \mathrm{H}_{2(\mathrm{~g})}+2 \mathrm{OH}^{-} \\
2 e_{(\mathrm{aq})}^{-}+\mathrm{H}^{+} \rightarrow \mathrm{H}+2 \mathrm{OH}^{-}
\end{gathered}
$$

The relative importance of these reactions depend upon concentration of solvated electrons, presence of solvated electron scavengers, temperature, $\mathrm{pH}$, and in some cases the presence of dissolved gases. For example, in the presence of dissolved oxygen, it was found that solvated electrons can combine with dissolved $\mathrm{O}_{2}$ to form $\mathrm{O}_{2}^{--}$as well [61]. The effects of plasma specific conditions on the lifetime and reactions of solvated electrons need further investigation.
The energy of an electron striking the surface is most important to those processes that have significant threshold energies. These interfacial and liquid phase reactions will most likely differ from gas-phase reactions because molecules in the liquid state more strongly interact with each other and multi-body processes will dominate over two-body reactions. Reaction products at the interface may become either gas-phase or liquid-phase species, and depending on the outcome, they will have very different impacts on the subsequent chemistry [211].

Over the past decade, our understanding has improved of the structure and dynamics of the hydrated electron, $e_{(\mathrm{aq})}^{-}$ through a combination of cluster experiments, the development of photoelectron spectroscopy of liquid micro-jets, and sophisticated simulations. A characteristic feature of $e_{(\mathrm{aq})}^{-}$is its strong $\left(\sigma_{298 \mathrm{~K}}=8.6 \times 10^{-17} \mathrm{~cm}^{2}\right)$ [212] optical absorption spectrum centered at $720 \mathrm{~nm}$ which has a distinctive shape with a Gaussian red edge and a Lorentzian blue edge [213, 214]. The reactivity of the hydrated electron has been extensively studied due to its role in radiation chemistry [184, 215]. Several laboratories have reported that the vertical detachment energy of the hydrated electron is $3.3 \mathrm{eV}$ using photoelectron spectroscopy of a water micro-jet [202, 216-218]. This result agrees with extrapolations of cluster experiments [214, 219] which, analogous to solid-state physics, have provided a view of $e_{(\mathrm{aq})}^{-}$as a defect state lying within the band-gap of water [220]. Calculations indicate that a large part of the electron distribution occupies a dynamic cavity formed by approximately 4 water molecules, while a roughly equal contribution protrudes out of this cavity including a diffuse tail that extends beyond the first solvation shell [221].

Assessments of the solvation of the hydrated electron at the water/air interface have relied on sophisticated simulations [208, 222, 223], second harmonic generation spectroscopy [224] and photoelectron spectroscopy of liquid micro-jets [225]. An early study observed unexpected weakly-bound surface electrons [226], akin to clusters [219]. The highest surface selectivity is achieved through second harmonic generation spectroscopy. This diagnostic has shown the dynamics of the interfacial electron to be similar to those in the bulk, thereby suggesting that at the interface, the electron can be regarded as being fully solvated. Other experiments indicate that $e_{(\mathrm{aq})}^{-}$resides within $\approx 1 \mathrm{~nm}$ of the surface [224]. This view is supported by calculations showing that a free electron at the water surface solvates within a few picoseconds to form a hydrated electron [208, 223]. From the perspective of a low energy electron approaching a water surface, the solvation dynamics to produce a fully solvated $e_{(\mathrm{aq})}^{-}$are expected to be very fast. Nevertheless, a fraction $(\approx 10 \%)$ of the density at the water/air interface may protrude into the vapor, suggesting that the reactivity of $e_{(\mathrm{aq})}^{-}$towards vapor-phase species may not be negligible.

In an atmospheric plasma with an aqueous anode, a large flux of electrons is incident onto the water-air interface, likely leading to a high interfacial concentration of $e_{(\mathrm{aq})}^{-}$, as recently measured for a DC glow discharge [199]. These electrons are both highly reactive and have an anomalously high diffusion constant, $D_{298 \mathrm{~K}}=4.9 \times 10^{-5} \mathrm{~cm}^{2} \mathrm{~s}^{-1}$. Many other sources 
of reactivity are also generated by the discharge, including highly reactive radicals and photons. The radiation chemistry community has evaluated many of these reactions [184, 215]. For example, ${ }^{\bullet} \mathrm{OH}$ is produced in large quantities near the interface. Given that the $e_{(\mathrm{aq})}^{-}+{ }^{\bullet} \mathrm{OH} \rightarrow \mathrm{OH}^{-}$reaction has a rate constant of $k_{300 \mathrm{~K}}=3 \times 10^{10} \mathrm{M}^{-1} \mathrm{~s}^{-1}$, efficient production of the $\mathrm{OH}^{-}$anion may be expected. Rates for the reaction of $e_{(\mathrm{aq})}^{-}$with most other relevant species have been similarly determined in water radiolysis [184]. However, it is unclear whether rate coefficients at the water/air interface are the same as in the bulk. The effect of strong local electric fields on interfacial electrons similarly requires further investigation. Electron collisions with clusters of two and more molecules of water have been analyzed using $R$-matrix methods [227, 228]. Additional insights may be obtained from gas-phase experiments on electron attachment to water clusters performed under cryogenic conditions. Due to the reactive nature of $e_{(\mathrm{aq})}^{-}$with most other species that are produced at the interface, it is likely that its density will not remain high. While the lifetime of solvated electrons is short compared to even short-lived radicals such as ${ }^{\bullet} \mathrm{OH}$, this short lifetime does not imply solvated electrons are not important. They are an important precursor for other reactive species and could play an important role in plasma-induced chemistry for reactions at the plasma-liquid interface.

\subsection{Plasma electrochemistry}

Electrochemical reactions can occur in plasma contacting liquids since classical reduction-oxidation reactions are likely in such systems. Electrode-mediated chemical processes such as electrolysis typically occur at solid-(electrode)-liquid interfaces. Classical electrochemical reactions at solid-liquid electrodes often follow and are generally limited by Faraday's law whereby the reaction yields are proportional to the current. Faraday's constant specifies the charge of one mole of electrons and Faraday's law assumes a 100\% current efficiency based upon the stoichiometry of the given electrochemical reaction. (See [229] for a more extensive analysis of the thermodynamics of water electrolysis reactions.) In many electrochemical reactions the current efficiency is less than $100 \%$. In early work on glow discharges and contact glow discharge electrolysis in liquids, Hickling [3, 230-232] demonstrated current efficiencies larger than $100 \%$ indicating that electrons from the plasma can participate in more than one reaction in series or additional radical induced reactions account for the increase in current efficiency (see figure 11). Current efficiencies larger than $100 \%$ have also been reported for electrical discharges in water [233]. Some cases reported less than $100 \%$ efficiency [234]. While energies of electrons in non-thermal plasmas are $0.1-10 \mathrm{eV}$, the temperature of electrons in classical electrolysis at ambient temperature $(298 \mathrm{~K})$ is $0.025 \mathrm{eV}$. Therefore there can be significant differences between classical electrochemical reactions and plasma chemical reactions. There is a growing field of plasma electrochemistry for plasma-solid interfaces to account for these differences [235, 236]. For example, in an argon plasma over a water surface direct electrolysis reactions such as $2 \mathrm{H}^{+}+2 e^{-} \rightarrow \mathrm{H}_{2(\mathrm{~g})}$ can be significant [237].

Another motivation to consider plasma electrochemistry arises from the electrical point of view that the plasma behaves like a metal electrode where the work-function has been elevated to the vacuum level. Depending on the specific coupling (DC, floating ground or AC) different mechanisms can take place but electron transfer occurs in a very similar way as in an electrochemical metal-liquid interface. However, different from a standard electrochemical interface, the electron energy needs to be included at the plasma-liquid interface. While the classical theory of plasma sheaths is the same as that in electrolytes (Debye-Huckel) chemical reactions may be different due to the higher energy of electrons in plasmas leading to different collisional cross sections.

\section{Photon-induced interactions}

\subsection{Introduction}

A significant fraction of the power dissipated by plasmas in gas/liquid environments is converted into ultraviolet (UV) light which may induce photochemical reactions [42]. The importance of UV radiation on the plasma produced chemistry in liquid is strongly dependent on the type of electrical discharge, the contact of the plasma with the liquid, and the gas composition. Photons most likely cross the plasma liquid interface unhindered other than by the change in index of refraction at the interface. The absorption of UV and vacuum ultraviolet (VUV) photons by water and impurities is well understood and characterized in the context of advanced oxidation processing (AOP) in water purification. There is a large knowledge base for plasma-liquid modeling that can be transferred from the AOP community in the context of removing pollutants from water [239, 240]. This knowledge base includes the photolysis of water and impurities [241, 242], radiation transport [243] and rate coefficients for reaction of reactive oxygen species (ROS) with organic and molecular species [184]. Synergetic effects of photochemistry and sonochemistry are also investigated [244]. This section provides an overview of VUV and UV induced reactivity in aqueous solutions.

\subsection{UV and VUV generation}

UV radiation is one of the principal forms of power dissipated from high-current electrical discharges in water [245-248]. Underwater plasmas initiated by exploding wires can transfer up to $28 \%$ of their energy into UV radiation [249]. The radiant power of the emitted UV radiation (190-280 nm) from pulsed streamer discharges in water ( $1 \mathrm{~J} /$ pulse) depends on the conductivity of the solution and can reach tens to hundreds of Watts during the pulse. This power corresponds to a UV radiation intensity of $0.1-10 \mathrm{~mW} \mathrm{~cm}{ }^{-2}$. In one experiment, the $190-280 \mathrm{~nm}$ photon production $J$ was related to the discharge power $P(\mathrm{~W})$ as $J=44.33 P^{2.11}$ (quanta/pulse) [250]. The absolute UV light emission from atmospheric pressure 
gas phase plasmas is significantly lower compared to those produced by underwater plasmas, although the fluence and intensity vary with the type of reactor, gas composition, and other conditions [251]. UV emission from typical air plasma discharges are mostly at UVA $(320 \mathrm{~nm}-400 \mathrm{~nm})$, and UVB $(280 \mathrm{~nm}-320 \mathrm{~nm})$ wavelengths with intensities below $50 \mu \mathrm{W}$ $\mathrm{cm}^{-2}$ [252]. No significant emission was observed in the UVC $(100 \mathrm{~nm}-280 \mathrm{~nm})$. However, plasmas in direct contact with water are expected to deliver both UV and VUV fluxes on the liquid surface. The addition of water vapor leads to emission from $\mathrm{OH}$ (at 284 and $309 \mathrm{~nm}$ ) [253, 254]. Dielectric barrier discharges (DBDs) are used for efficiently producing VUV excimer radiation with efficiencies up to $60 \%$ for $\mathrm{Xe}_{2}$ excimer emission. Similarly, plasma jets operating in atmospheric pressure Ar can emit significant amounts of excimer radiation [255]. This excimer radiation can then be transported several $\mathrm{cm}$ from the nozzle of the plasma jet [256] where photochemical reactions can take place. UV radiation is therefore an important process in plasmas interacting with water. An example of the VUV and UV emission spectra of an argon based plasma jet is shown in figure 12 .

\subsection{VUV radiation}

VUV radiation with $\lambda=75-185 \mathrm{~nm}$ is particularly efficient in inducing chemical reactions. Since the absorption cross section of VUV photons by water molecules is large [258-260], the effect of VUV photolysis in practice occurs within a thin layer near the gas-liquid interface. The absorption coefficient of liquid water at $\lambda<170 \mathrm{~nm}$ is $10^{3}-10^{5} \mathrm{~cm}^{-1}$ [259], which means that $90 \%$ of the radiation is absorbed by a water layer with a thickness of $10^{-3}-10^{-5} \mathrm{~cm}$. At a wavelength of $185 \mathrm{~nm}$ the penetration depth is about $0.1 \mathrm{~cm}$ [261]. The absorption coefficient of saturated water vapor at a temperature of $24{ }^{\circ} \mathrm{C}$ $(P=3 \mathrm{kPa})$ is $1-10^{-1} \mathrm{~cm}^{-1}$ [260], which results in the absorption of $90 \%$ of the radiation in a vapor layer having a thickness of several centimeters.

Absorption of VUV irradiation by liquid water with dissolved gases such as $\mathrm{O}_{2}$ results in dissociation of water and $\mathrm{O}_{2}$ molecules, and formation of hydroxyl radicals, hydrated electrons, and oxygen atoms (ground state and excited). Some of the major reactions and their quantum yields for common VUV wavelengths are reported in [241, 262-267]:

\begin{tabular}{|c|c|c|c|}
\hline Reaction & $\lambda(\mathrm{nm})$ & $\begin{array}{l}\text { Quantum } \\
\text { yield }\end{array}$ & \\
\hline \multirow[t]{3}{*}{$\mathrm{H}_{2} \mathrm{O}_{(\mathrm{aq})}+h \nu \rightarrow \bullet \cdot \mathrm{OH}+\mathrm{H}^{\bullet}$} & 124 & 0.93 & \multirow{3}{*}{ (8) } \\
\hline & 147 & 0.65 & \\
\hline & 185 & 0.3 & \\
\hline $\begin{array}{l}\mathrm{H}_{2} \mathrm{O}_{(\mathrm{aq})}+h \nu(<200 \mathrm{~nm}) \rightarrow \\
{\left[e^{-}, \mathrm{H}_{2} \mathrm{O}^{+}\right]}\end{array}$ & 124 & 0.10 & \multirow{3}{*}{ (9) } \\
\hline \multirow{2}{*}{$\begin{array}{l}{\left[e^{-}, \mathrm{H}_{2} \mathrm{O}^{+}\right]+\mathrm{H}_{2} \mathrm{O}_{(\mathrm{aq})}} \\
e_{(\mathrm{aq})}^{-}+{ }^{\bullet} \mathrm{OH}+\mathrm{H}_{3} \mathrm{O}^{+}\end{array}$} & 147 & 0.07 & \\
\hline & 185 & 0.03 & \\
\hline \multirow[t]{2}{*}{$\mathrm{H}_{2} \mathrm{O}_{(\mathrm{g})}+h \nu \rightarrow{ }^{\bullet} \mathrm{OH}+\mathrm{H}^{\bullet}$} & $105-145$ & 0.89 & \multirow{2}{*}{$(10 a)$} \\
\hline & $145-185$ & 1 & \\
\hline \multirow{2}{*}{$\mathrm{H}_{2} \mathrm{O}_{(\mathrm{g})}+h \nu \rightarrow \mathrm{H}_{2}+\mathrm{O}\left({ }^{1} \mathrm{D}\right)^{\bullet}$} & $105-145$ & 0.11 & \multirow{2}{*}{$(10 b)$} \\
\hline & $145-185$ & 0.01 & \\
\hline $\mathrm{O}_{2}+h \nu \rightarrow 2^{\bullet} \mathrm{O}$ & $139-175$ & 1 & (11) \\
\hline
\end{tabular}

While these reactions are often the main reactions, 30 reactions have been identified in total [241].

Photon transport depends on the absorption cross section and the density of the absorbers. From atmospheric chemistry the cross section for reaction (10) spans $10^{-17}-10^{-18} \mathrm{~cm}^{2}$ [265]. Excess photon energy can be dissipated in the kinetic energy of the fragments $[266,267]$. The ${ }^{\circ} \mathrm{OH}$ formed by reaction (8) emerges in different electronic, vibronic and rotational states depending on the absorbing wavelength [268]. The products of reactions (8) and (9) in water initiate the formation of secondary species such as $\mathrm{H}_{2},{ }^{\bullet} \mathrm{OH}^{-}, \mathrm{O}^{\bullet-}, \mathrm{H}_{2} \mathrm{O}_{2}$, $\mathrm{O}_{2}^{--}, \mathrm{HO}_{2}^{\bullet}, \mathrm{HO}_{2}^{-}, \mathrm{O}_{3}^{-\bullet}, \mathrm{O}_{2}[269,270]$, as modeled in [62, 271].

The main disadvantage of VUV photolysis is the very thin water layer in which these radicals are formed which produces high local concentrations of radicals which in turn may lead to recombination and so a lower overall reactivity. For example, from modelling the concentration of ${ }^{\bullet} \mathrm{OH}$ due to $172 \mathrm{~nm}$ VUV radiation is $10^{14} \mathrm{~cm}^{-3}$ for liquid water $\left(100 \mathrm{~mW} \mathrm{~cm}{ }^{-2}\right)$ and $10^{13} \mathrm{~cm}^{-3}$ for water vapor $\left(10 \mathrm{~mW} \mathrm{~cm}^{-2}\right)$ [272]. Direct photolysis of organic compounds may also occur in the VUV region [273].

Due to the high absorption coefficients of VUV radiation, large effects can occur in small volumes, which produce extreme heterogeneity between the irradiated volume and the non-irradiated bulk solution [261]. The rate of induced bulk chemical reactivity strongly depends on the rate of mass transfer into and out of this small volume. These trends were confirmed using computational fluid dynamics (CFD) modeling [240]. Dissolved molecular oxygen in general plays an important role because it can react with short-lived hydrogen, carbon radicals (from fragments of organic molecules) and, to a smaller extent, with solvated electrons, resulting in the formation of longer-lived peroxyl radicals in the presence of organic molecules. Thus, diffusion of oxygen into the irradiated volume also may become a rate limiting factor for the degradation of organic material.

\subsection{UV radiation}

UV radiation with wavelengths greater than $185 \mathrm{~nm}$ penetrates into the bulk of an aqueous solution where it can produce photochemical reactions with molecules other than water such as naturally occurring organic compounds, organic micropollutants, nitrates and nitrites. Following absorption of UV radiation by organic compounds, the molecule may dissociate and produce smaller organic compounds [239, 274]. There are large differences in photodissociation cross sections of organic compounds and differences in their dissociation products [274, 275].

Inorganic compounds also photodissociate following absorption of UV radiation which, in an aqueous environment, immediately lead to secondary reactions. In these reactions oxygen atoms and hydroxyl radicals play important roles [256, 276]:

$$
\begin{aligned}
& \mathrm{NO}_{2}^{-}+h \nu \rightarrow{ }^{\circ} \mathrm{NO}+\mathrm{O}^{\cdot-} \\
& \mathrm{NO}_{3}^{-}+h \nu \rightarrow{ }^{\cdot} \mathrm{NO}_{2}+\mathrm{O}^{\cdot-}
\end{aligned}
$$




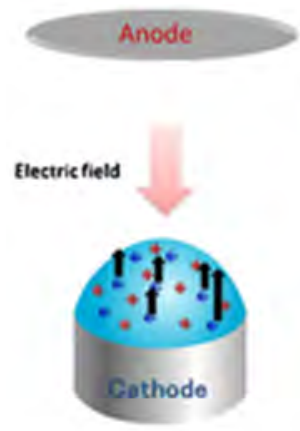

(a)

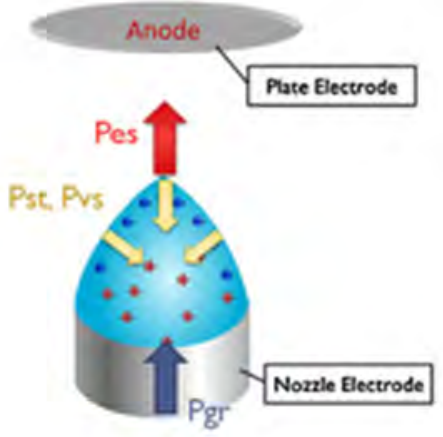

(b)

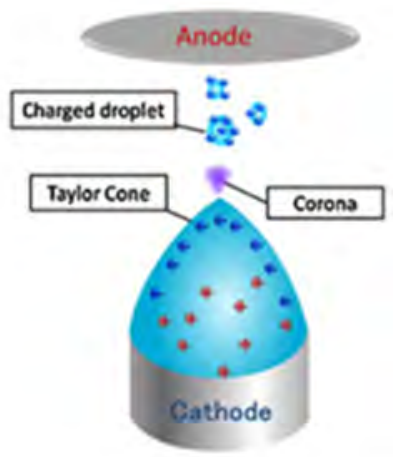

(c)

Figure 13. The formation of a Taylor cone: (a) initial state; (b) formation of cone; (c) corona discharge at the tip of the cone. Reproduced with permission from [296], copyright 2014 JSAP.

$$
\begin{gathered}
\mathrm{NO}_{3}^{-}+h \nu \rightarrow \mathrm{NO}_{2}^{-}+\mathrm{O}^{\bullet} \\
\mathrm{O}^{\bullet}+\mathrm{H}_{2} \mathrm{O} \rightarrow 2 \cdot \mathrm{OH} \\
\mathrm{O}^{\bullet-}+\mathrm{H}_{2} \mathrm{O} \rightarrow \cdot{ }^{\bullet} \mathrm{OH}+\mathrm{OH}^{-} \\
2 \mathrm{NO}_{2}^{\bullet}+\mathrm{H}_{2} \mathrm{O} \rightarrow \mathrm{NO}_{2}^{-}+\mathrm{NO}_{3}^{-}+2 \mathrm{H}^{+}
\end{gathered}
$$

The reactive species formed in these processes may further react with organic compounds. Processes that involve hydroxyl radicals fall under the category of AOP. Hydroxyl radicals have rate coefficients near the diffusion limit (the equivalent of gas-kinetic rate coefficients in the gas phase), and non-selectively react with numerous organic molecules. There are several ways to initiate AOP, an example being UVA photolysis of nitrites produced by plasma that is effective in inactivating $E$. coli $[79]$.

A well-known AOP based on UV is the $\mathrm{UV} / \mathrm{H}_{2} \mathrm{O}_{2}$ process in which $\mathrm{H}_{2} \mathrm{O}_{2}$ is added to water. Hydroxyl radicals are the product of photolysis of $\mathrm{H}_{2} \mathrm{O}_{2}$ by $\mathrm{UVC}$ radiation,

$$
\mathrm{H}_{2} \mathrm{O}_{2}+h \nu \rightarrow 2^{\circ} \mathrm{OH}
$$

The hydroxyl radicals initiate a reaction which provides a high quantum yield of the photolysis of $\mathrm{H}_{2} \mathrm{O}_{2}$ :

$$
\begin{gathered}
\cdot \mathrm{OH}+\mathrm{H}_{2} \mathrm{O}_{2} \rightarrow \mathrm{H}_{2} \mathrm{O}+\mathrm{HO}_{2}^{\cdot} \\
\mathrm{HO}_{2}^{\cdot}+\mathrm{H}_{2} \mathrm{O}_{2} \rightarrow \mathrm{O}_{2}+\mathrm{H}_{2} \mathrm{O}+{ }^{\bullet} \mathrm{OH}
\end{gathered}
$$

This process is important in the production of $\mathrm{H}_{2} \mathrm{O}_{2}$ by inliquid plasmas. For example, the yield of $\mathrm{H}_{2} \mathrm{O}_{2}$ produced by in-liquid discharges decreased as the conductivity of the liquid of the increased due to the increasing photolysis of $\mathrm{H}_{2} \mathrm{O}_{2}$ [250].

Another AOP based on $\mathrm{UV}$ is the $\mathrm{UV} / \mathrm{O}_{3}$ process which relies on the photolysis of ozone in the range $200-280 \mathrm{~nm}$. The photolysis of $\mathrm{O}_{3}$ produces the excited state $\mathrm{O}\left({ }^{1} \mathrm{D}\right)^{\bullet}$ which in turn reacts with water to produce hydroxyl radicals [277].

$$
\begin{aligned}
& \mathrm{O}_{3}+h \nu \rightarrow \mathrm{O}_{2}+\mathrm{O}\left({ }^{1} \mathrm{D}\right)^{\bullet} \\
& \mathrm{O}\left({ }^{1} \mathrm{D}\right)^{\bullet}+\mathrm{H}_{2} \mathrm{O} \rightarrow 2^{\bullet} \mathrm{OH}
\end{aligned}
$$

\subsection{Photocatalytic processes}

Heterogeneous photochemical reactions utilize photocatalysts such as $\mathrm{TiO}_{2}$. Absorption of UV radiation causes the formation of both conduction band electrons $\left(e^{-}\right)$and valence band holes $\left(h^{+}\right)$[278, 279]. The electrons induce reduction reactions, whereas the holes can cause oxidation reactions. In the presence of oxygen, hydroxyl radicals may be formed, and so the combination of UV and photocatalysts can be considered an AOP. The wavelengths of the photons that can be absorbed and activate the photocatalysts depend on the crystal structure (e.g. anatase or rutile in $\mathrm{TiO}_{2}$ ) and on the presence of other elements or compounds at the surface of the crystal or in solution [280]. The effectiveness of the process can often be improved by adding $\mathrm{H}_{2} \mathrm{O}_{2}$ [280] or ozone [281]. UV radiation from pulsed corona discharges has been used to induce photocatalytic processes in water [282-284].

\section{Fluid dynamics, heat and mass transport}

\subsection{Introduction}

Thermodynamics and transport phenomena in chemically reactive, ionized, multiphase fluid media are complex processes. As texts are devoted to even subsets of the topics addressed here, our intent is to only provide an introduction and to identify some of the key research challenges in fluid dynamics, heat and mass transport during the interactions between chemically reactive non-thermal plasmas and liquids at atmospheric pressure.

The theoretical description of fluid phases at atmospheric pressure generally assumes that the fluid is at local thermodynamic equilibrium (LTE). That is, all thermodynamic properties of the fluid such as internal energy and entropy, transport coefficients and rates of reaction are determined by the instantaneous and local values of temperature, pressure, and chemical composition. This assumption is always violated when dealing with non-equilibrium ionized gas-phase plasmas since electrons in the gas phase are not at thermodynamic equilibrium with the heavy particles. Electrons usually have mean thermal energies of a few eV compared to 


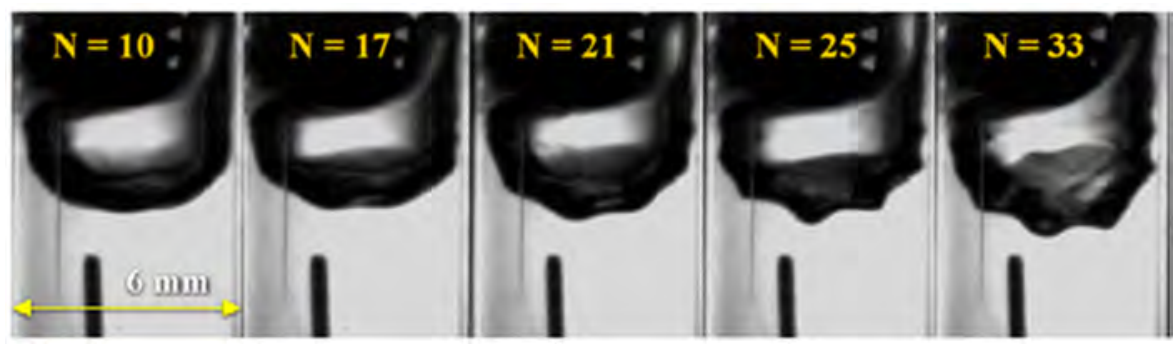

Figure 14. Streamer driven capillary waves on surface of electrode attached bubble. $N$ refers to number of streamer strikes. Reproduced with permission from [304].

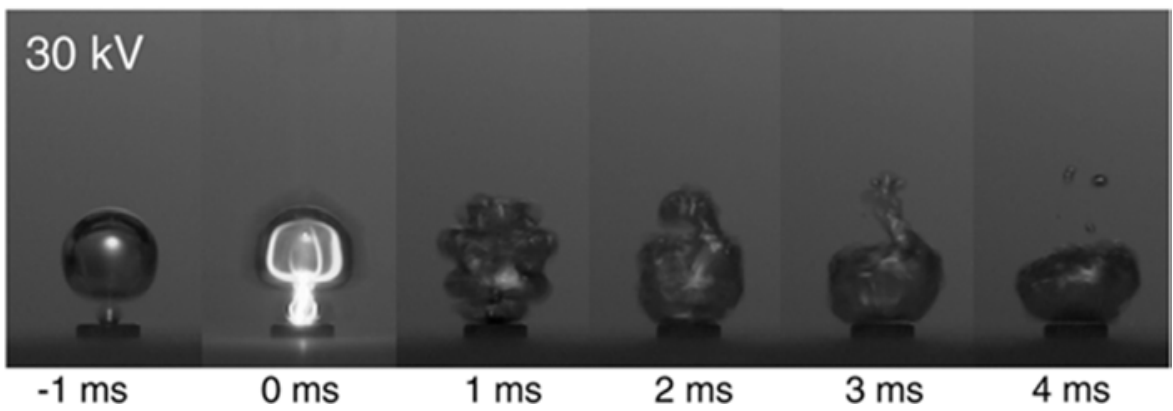

Figure 15. Bubbles after a discharge created by a $30 \mathrm{kV}$ pulse applied to a needle electrode inside $\mathrm{N}_{2}$ gas. Images show the bubble before, during and after the discharge. Reproduced with permission from [69], 2011 copyright IOP Publishing.

the typically room temperature neutral species. LTE is still generally a good starting point for the majority neutral species, although important exceptions (e.g. vibrationally excited molecules or hot atoms, created by dissociative excitation) are common. Plasma electrons and ions generally have low mole fractions relative to neutral species. Their low concentrations enable those species to be treated separately from the fluid with appropriate collisional terms to account for transfer of energy and momentum with the neutral fluid with no significant loss of generality.

When more than one phase is present, for example when a gas phase is in contact with a liquid phase, there are wellestablished methods of treating the system. The most common assumption is that LTE applies at the interface for thermal and mechanical properties. With this approximation, the temperatures of the two phases are assumed to be equal at the interface and the phase boundary is at mechanical equilibrium (i.e. forces are balanced). The chemical compositions of each phase at the phase boundary follow from either bulk phase equilibrium properties (solubility or vapor pressure) or finite rate chemical reactions.

The solution properties may be non-ideal in which the thermodynamic properties are non-linear functions of the concentration of all other species present. The properties of non-ideal mixtures cannot be determined by mole fraction-weighted sums of pure component properties. This adds considerable complexity to the analysis and in some cases simpler approaches may suffice. For example, the gas will behave ideally in non-thermal atmospheric pressure plasmas for all but extreme conditions where, for example, excited states or ions have significant mole fractions.
Important aspects of fluid dynamics, heat and mass transport in the context of plasma-liquid interactions at atmospheric pressure are discussed in this section. The treatment largely follows conventional transport theory valid for any reacting gas/liquid system with flows being either laminar or turbulent. In this regard, interfacial mixing, stability and the nature of the interface are important. Special cases include: (a) two phases separated by a mostly stable interface with a well-defined area, (b) a continuous gas phase and discontinuous liquid phase (e.g. droplets of liquid immersed in a gas flow), (c) a continuous liquid phase with discontinuous gas phase (e.g. bubbles of gas, perhaps containing plasma, immersed in liquid), and (d) a completely turbulent, strongly disordered mixture of the two phases (e.g. when a turbulent gas flow strongly mixes with a liquid). Chemical reactions can have a large effect on the system as these reactions can change transport properties and produce changes in enthalpy.

Phase changes such as evaporating liquid and condensing or dissolving vapor are coupled to the Joule heating of electric discharges in contact with liquids. Ions and electrons play important roles in the chemical composition of the liquid due to the reactions they initiate. They are also important to interfacial stability through surface charging, electric fields and currents at the liquid-gas surface. For example, Taylor cones can form on charged liquid surfaces, and charged droplets and bubbles can have complex dynamic behavior which influences interfacial transport phenomena. The coupling between the ionized or plasma nature of the gas phase and the ionized or electrolyte nature of the liquid phase is a potentially important issue. Electric fields driving currents in each phase will in general affect the transport processes in each phase and at the interface. 


\subsection{Coupled plasma-liquid surface deformation}

External forces associated with plasma production can significantly perturb the surface of the liquid at the plasma-liquid interface. Energetic particles at the interface, both charged and uncharged, bombard the surface as do photons. Electric fields resulting from the applied voltage and space charge can physically distort the surface [285-292]. Such electric field initiated distortions of the surface can lead to electric field enhancement which in turn leads to breakdown. The combination of these processes can initiate oscillations or instabilities at the interface as well as drive in-volume processes such as enhanced mixing, convection, and the dissipation of energy into the liquid. These instabilities may explain enhanced diffusion leading to in-volume mixing in static DBD-liquid reactors [293].

The interaction of streamers at the interface can also be a source of acoustic energy or even shock waves. These acoustic processes can drive radical production within the bulk liquid. Such acoustic energy is the basis of sonochemistry [294]. The non-linear surface wave-inducing effects of electric discharges above water have been investigated and analyzed by Robinson et al [295].

The formation of a Taylor cone at the gas plasma-liquid interface is schematically shown in figure 13. Taylor cones can form on the surface of an electrolyte liquid placed between two electrodes [296]. The electric field in the gas phase induces ionic charges in the liquid to form a cone-like shape on the free surface, leading to the emission of small charged droplets, in this case traveling from the liquid on the cathode surface towards the anode surface. These droplets can become part of a discharge between the electrodes. Taylor [297] first outlined the conditions leading to the formation of cones. The interface must be an equipotential surface and have a shape so that the electric field-induced force balances the surface tension induced by the curved liquid surface. The dynamics of a charged free liquid surface manifests itself in a variety of phenomena in nature and technology. For example, this phenomenon is important in the electrospinning of fibers and electrosprays (e.g. in mass spectrometry). Extensive analyses have been performed on the general physics of liquid jets and their stability [298]. In the context of plasma-liquid interactions, Bruggeman et al showed that a water surface can be deformed by the application of an electric field between a conducting pin and the water surface [291]. This deformation was followed by gas breakdown and plasma formation. It is likely that water vapor will play some role in the breakdown process under these conditions. Schwartz et al report observations of ejecting of droplets and solution plumes at the plasma-liquid interface for solution-cathode glow discharges [299].

A different kind of plasma-gas-liquid surface coupling occurs when a corona discharge in the gas phase produces an electric field-induced flow of ions that couple by momentum transfer collisions to induce neutral gas convection, socalled ionic wind. This gas convection can be seen dimpling of the surface of the liquid [63]. The shape of the water surface is controlled by a balance between the surfacenormal pressure force caused by the stagnation pressure of the gas, the fluid hydrostatic pressure, the gas-liquid shearing force and the surface tension force. Similar phenomena occur when a plasma jet impinges on a liquid. The water subjected to a gas jet will dimple, splash or the gas will penetrate depending on the magnitude of the gas jet momentum [300]. For modest gas flows, the water surface is dimpled and only weakly disturbed [301]. In the case of a turbulent gas jets, interactions with liquid surfaces would likely lead to stronger disruption of the surface and ultimately, gasliquid turbulent mixing [302].

\subsection{Plasma coupled with gas bubbles}

Instabilities at the plasma-liquid interface have been observed in gas bubble plasmas in liquids. Here plasma is introduced into the liquid through the breakdown of the gaseous content of the bubbles. The boundary of the bubble can be dynamically distorted due to acoustic, thermal, and electrostatic energy associated with plasma formation. Capillary wave excitation and subsequent propagation along the surface of a bubble has been observed in electrode-attached bubbles in which a streamer contacts the surface of the bubble, as shown in figure 14 [303]. Electric fields due to streamers inside the bubble are sufficiently strong relative to surface tension to perturb the boundary. Tachibana et al [69] showed how pulsed discharges within bubbles distort and disrupt the shape of the bubble. This disruption is shown in figure 15 for a bubble in $\mathrm{N}_{2}$ gas with $30 \mathrm{kV}$ applied to the electrode. The capillary waves produced at the surface of the bubble are a source of acoustic energy and can be transmitted to adjacent bubbles [288]. Ultrasound produced by these oscillations can drive sonochemical processes and so are important to understanding the interaction between plasma and bulk chemical changes in solution.

In the presence of a time varying electric field, bubbles can undergo distinct oscillation modes which can be described by spherical harmonics. These instabilities at the surface of the bubble can in turn locally distort the electric field leading to breakdown $[291,305]$. With immersed electrodes and a finite liquid conductivity, Joule heating can lead to instability at the surface of the electrode, leading to a phase transition and a vapor bubble [288, 306, 307]. If the applied field is sufficiently strong, then plasma can form.

\subsection{Plasma-induced liquid phase convection and mass transfer between gas and liquid phases}

Convection-or bulk fluid motion-in the liquid phase is an important process in distributing reactivity produced by the plasma at the plasma-liquid boundary. If the plasma is sustained in the form of a jet, streamer discharge or corona (producing the ionic wind), there is bulk gas convection associated with the plasma [308-310]. Shimizu et al [311] investigated the effects of a streamer discharge in air above a liquid surface and found that convection could be induced in the liquid with speeds of several $\mathrm{cm} \mathrm{s}^{-1}$, sufficient to dominate over diffusion. The induced liquid flow was caused by shear stress at the 
(a)

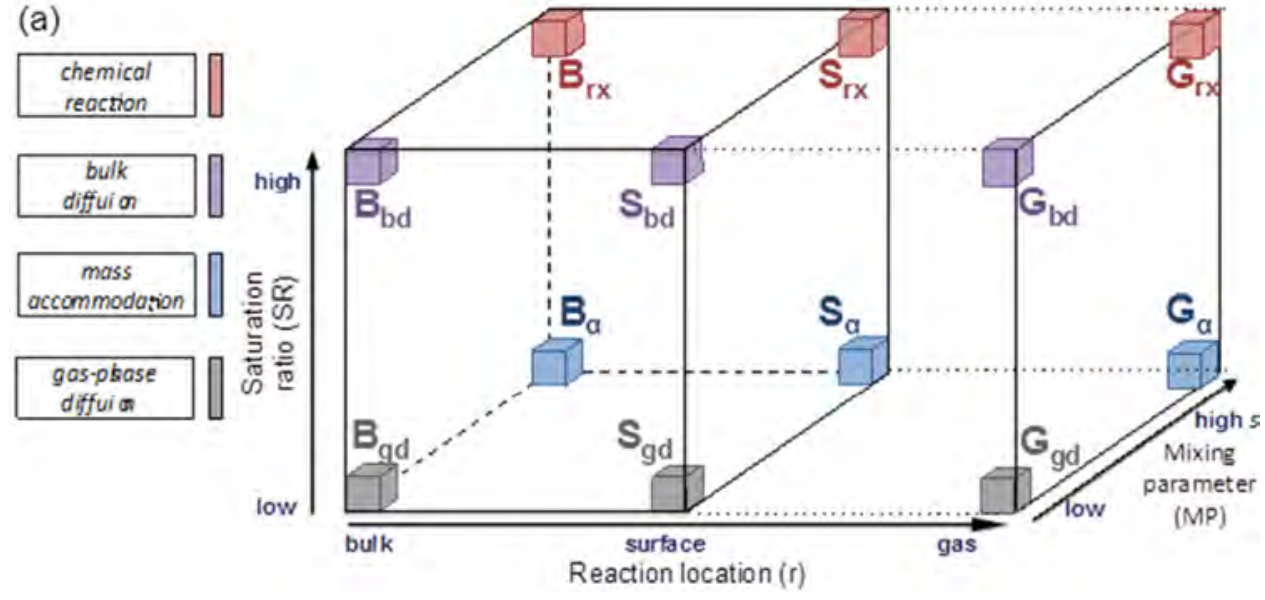

Figure 16. Kinetic regimes and limiting cases of gas uptake by aerosol particles or bulk liquid mapped onto the axes of a cuboid representing reaction location, saturation ratio, and mixing parameter. Horizontal edges of the cuboid (left to right) correspond to four regimes governed by chemical reactions, bulk diffusion, mass accommodation, or gas-phase diffusion. Each regime includes limiting cases characterized by a single rate-limiting process and a dominant reaction location (particle bulk, $B$; surface, $S$; gas phase, $G$ ). Reproduced with permission from [318].

interface by the moving gas [311], an important mechanism in many plasma-liquid systems. Other complexities and subtleties certainly occur, demonstrated by Ar plasma jet—liquid surface interactions imaged by van Rens et al [301]. Other mechanisms inducing liquid flow include thermally induced natural convection, Marangoni, electroosmotic and electrophoretic flows [312, 313]. Pressure gradients due to surface charging and corresponding induced flow can also occur. The latter is a surface flow induced by gradients in the gas-liquid surface tension. This could be caused by temperature gradients and/ or concentration gradients along the surface of the gas-liquid interface [314].

The plasma-induced liquid convection, discussed further below, is an important subset of the transfer of (generally relatively dilute) chemical species between fluid phases-either from the gas to the liquid or the reverse. This is a topic that has a large literature and is important in both technology and nature [315]. In order for species to transport between phases, several processes must occur in series. First, the species must be transported to the phase interfacial boundary-generally either by bulk fluid motion (i.e. convection) and/or diffusion. The species must then enter the other phase at the interface and be transported away from the interface. Chemical reactions that transform the composition of the mixture may occur at any of these steps. The methods used to quantitatively compute the overall rate of mass transfer can vary depending on the conditions and goals of the analysis. The most general approach is to compose a set of coupled transport equations, chemical kinetic expressions and thermodynamic relations. While this approach is the most general, and allows for the coupling between individual steps that can be important, it is also the most complex and difficult to solve and interpret. An alternative approach is to consider the individual processes as independent, each consisting of a driving force and associated coefficient, either a kind of conductivity or resistance. The overall rate calculation is then similar to linear electrical circuit analysis with series or parallel resistances and/or capacitances. This approach has been developed for analyzing

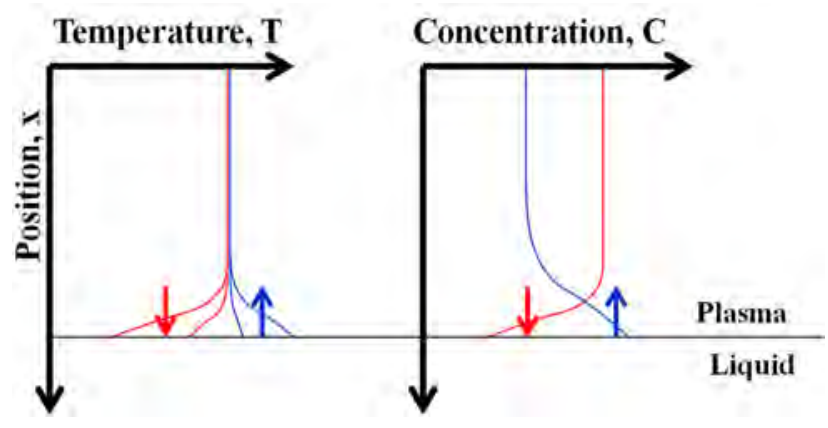

Figure 17. Schematics of temperature and concentration profiles above liquid in contact with plasma. The arrows indicate that both chemical species and energy can be transported in either direction depending on the local gradients.

transport phenomena into and out of atmospheric aerosols. Coupled transport and reaction phenomena are summarized in reviews in the atmospheric aerosol literature [156, 161, 316-319].

The problems that arise in the context of plasma-liquid interactions will not be identical to atmospheric aerosols, but there are many useful analogies that can be used to analyze the interfacial transport. For example, Berkemeier et al [317] considered a gas phase species $X$ moving from the gas phase to the aerosol surface and reacting with a compound $Y$ in the condensed phase. The overall rate is in general affected by one or more of the individual rate processes: the rate of mass transport of $X$ to the particle surface; rate of reaction between $X$ and $Y$ at the surface; diffusion rate of $X$ or $Y$ in the bulk of the particle; and reaction rate in the bulk region. Eight limiting cases were identified. One such example for reactions of particle $X$ and $Y$ at the surface has the supply of $X$ from the gas phase not limiting the process (so gas phase mass transfer is not limiting) and transport of $Y$ from the bulk is not limiting the process. In this case, the rate of reaction is controlled solely by reaction kinetics between $X$ and $Y$ at the surface. At each step, a dimensionless quantity relating competing 

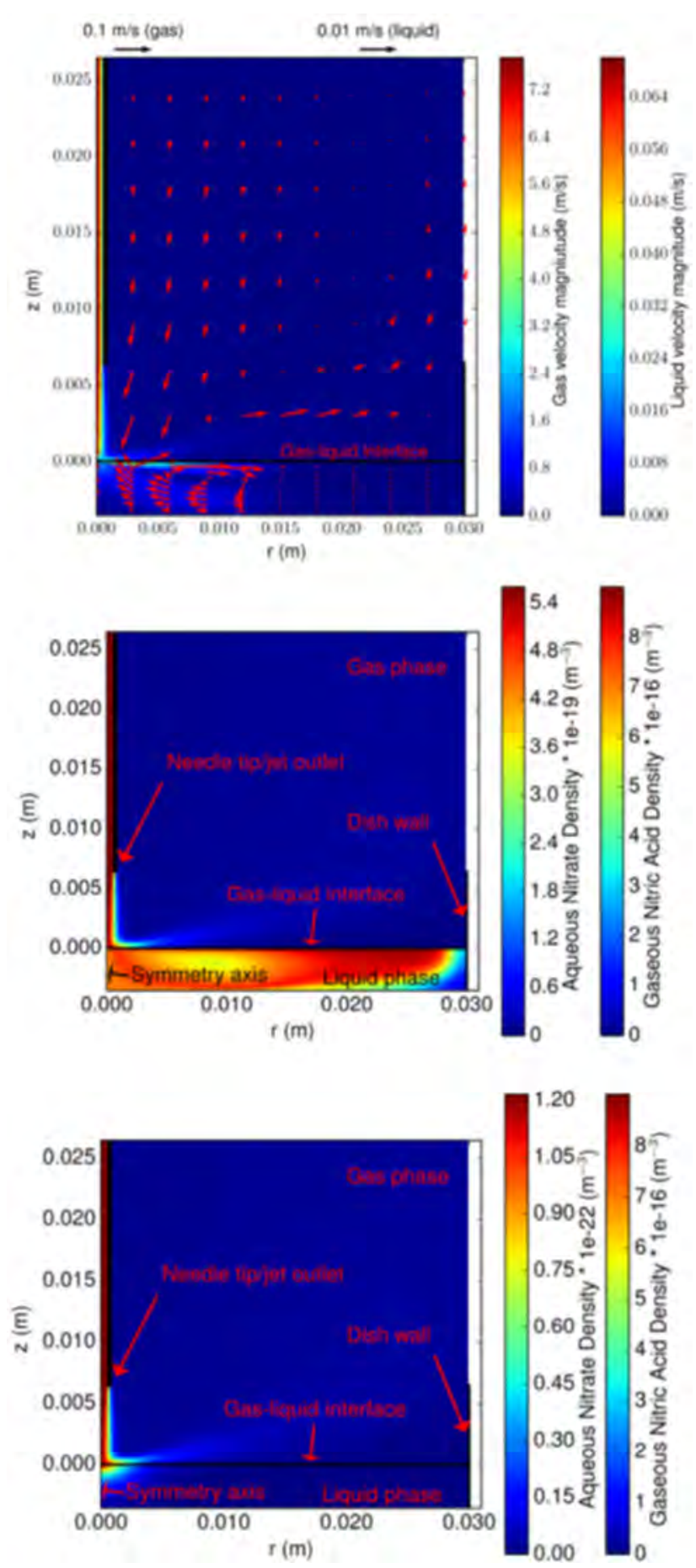

Figure 18. Model of the interaction of a reactive flow originating from the needle tip with relevant reactive species densities deduced from DBD plasmas. (top) The flow pattern in the gas and liquid phase. (middle) Distribution of the $\mathrm{HNO}_{3}$ density in the gas and liquid phase. (bottom) Distribution of the $\mathrm{HNO}_{3}$ density in the gas and liquid phase without liquid convection. Reproduced with permission from [323], copyright 2015 IOP Publishing.

rates is evaluated to determine if one of the competing rates is much larger than the others which then determines the limiting regime (see figure 16). This analysis, as described, does not necessarily address potentially more complex conditions at the bulk plasma-liquid interface. These conditions may include reaction mechanisms involving multiple chemical species and/or parallel or sequential reactions, heat transfer between phases, electrical effects in either phase, the possible role of convection in the bulk condensed phase, the possibility of unstable surfaces and the role of photons.

A critically important parameter in interfacial mass transfer is the ratio of gas and liquid concentrations of species at the interface, usually characterized by a parameter called the Henry's law coefficient, or $H$. There are many possible units for gas and liquid phase concentrations so the corresponding numerical values of $H$ will depend on these choices of units. Here, we use units of M/atm-liquid concentration of moles species per liter liquid divided by gas phase concentration expressed as the partial pressure in atm. For example, approximate $H$ values (at $298 \mathrm{~K}$ ) for species of interest to air plasmas are: $\mathrm{O}_{2}\left(1 \times 10^{-3}\right)$; ${ }^{\bullet} \mathrm{NO}\left(2 \times 10^{-3}\right) ; \mathrm{O}_{3}\left(1 \times 10^{-2}\right)$; $\mathrm{NO}_{2}\left(4 \times 10^{-2}\right) ; \mathrm{N}_{2} \mathrm{O}_{4}(1) ;{ }^{\bullet} \mathrm{OH}(25) ; \mathrm{HNO}_{2}(50) ; \mathrm{H}_{2} \mathrm{O}_{2}\left(10^{5}\right)$; $\mathrm{HNO}_{3}\left(10^{5}\right)$ [143]. The $H$ values differ by orders of magnitude and these differences can dominate rates of mass transfer in practice. Gases such as $\mathrm{O}_{2}, \mathrm{O}_{3}$ and ${ }^{\bullet} \mathrm{NO}$ have quite low values of $\mathrm{H}$ compared to $\mathrm{H}_{2} \mathrm{O}_{2}$ or $\mathrm{HNO}_{3}$, and they are much more difficult to transfer from the gas to the liquid (water) phase. The temperature dependence of activity coefficients used to determine $H$ is discussed in [320].

Another important factor in interphase mass transfer is the role of chemical reactions. If a gas phase species, such as $\mathrm{O}_{3}$, is absorbed into pure water with no reaction to convert ozone into another compound, the rate of mass transfer from the gas phase will be relatively slow as ozone is relatively hydrophobic. This is reflected by the relatively low value of $\mathrm{H}$ for $\mathrm{O}_{3}$ in water. However, if there is a compound in the water that rapidly reacts with $\mathrm{O}_{3}$ as soon as it enters the water, the rate of mass transfer of $\mathrm{O}_{3}$ from the gas phase could be much higher. The steady-state value of the ratio of liquid to gas phase concentrations (reflected in the value of $H$ ), arises because at steady-state, $\mathrm{O}_{3}$ enters and leaves the water at the same rate. If the rate of $\mathrm{O}_{3}$ leaving the water and re-entering the gas phase is negligible because $\mathrm{O}_{3}$ quickly reacts when entering the water, then $\mathrm{O}_{3}$ will only enter the water. The net rate of mass transfer may therefore dramatically increase when such reactive losses occur in the liquid phase. See, for example, [321, 322].

\subsection{Coupled heat and mass transfer at plasma-liquid boundaries}

An important consideration in plasma-liquid coupling are the ways that heat and mass will be transferred between plasma and liquid. Some of these concepts are illustrated in figure 17. Chemical species impacting the liquid surface will adsorb onto the surface with probability $\alpha_{\mathrm{s}}$, absorb in the particle bulk with probability $\alpha_{\mathrm{b}}$ and desorb from the surface with characteristic time $\tau_{\mathrm{d}}$ with the net sticking coefficient being $\gamma$ [156]. The plasma produces reactive species in the gas phase which diffuse, drift (as ions) and convect to the liquid surface where they can interact with the liquid phase by these same set of processes. The concentration profiles sketched in figure 17 above the liquid surface show that some species will enter the 
liquid phase (indicated by the arrow towards the liquid surface corresponding to a negative concentration gradient) and some species may desorb from the liquid phase and enter the gas phase.

The liquid convection induced by a plasma or gas flow can strongly alter the transport of chemical species in the liquid. For example, the plasma induced convection in the liquid produced by a streamer-like discharge is shown in figure 18 , for transport of $\mathrm{HNO}_{3}$. Diffusive speeds in the liquid phase are generally small relative to even very small convective currents in the liquid. The diffusive speed scales as diffusivity divided by characteristic length $(D / L)$, which is about $\left(10^{-5} \mathrm{~cm}^{2} \mathrm{~s}^{-1}\right) /$ $(1 \mathrm{~cm})$ or $\approx 10^{-5} \mathrm{~cm} \mathrm{~s}^{-1}$ in the bulk liquid. Even a relatively slow liquid flow velocity of $<1 \mathrm{~mm} \mathrm{~s}^{-1}$ is much higher than the diffusive speed. Convective transport (bulk motion of the liquid) tends to strongly dominate diffusive transport (due to random thermal motion) in the bulk liquid. This corresponds to a large Peclet number (i.e. the ratio of advective to diffusive transport). This may not necessarily be true at the interface of the plasma and liquid where there can be severe concentration gradients which enhance diffusive transport by many orders of magnitude.

Vapor from the liquid will be produced by evaporation at the plasma/gas-liquid interface since the plasma generally transfers heat to the liquid surface. The gas temperature profiles sketched in figure 17 are meant to show that heat may be transferred to or from the liquid surface. If, for example, the plasma transfers more energy to the liquid surface than can be transferred deeper into the liquid, to the evaporating water or to the bounding liquid container, the gas temperature at the gas-liquid boundary will be higher than the bulk gas temperature. Thermal conduction may therefore transfer heat either to or from the gas-liquid interface into the gas.

It is more common that the gas temperature in the plasma will be higher than that of the water and thermal conduction will transfer heat from the gas to the liquid. In general, the transfer of energy from the gas to the liquid (either in the form of thermal conduction of heat or in the form of ionized, vibrationally, electronically excited molecules or dissociated molecules) will cause some liquid to evaporate into the gas phase if the gas is not already saturated with the vapor. This is sometimes termed simultaneous heat and mass transfer because the rate of heat transfer to the liquid from the gas is balanced by and coupled to the rate of mass transfer of water vapor from the gas-liquid interface into the bulk gas phase [315]. If all the heat needed to evaporate the liquid into the gas comes from the gas itself (a common situation), then the rate of heat transfer to the surface of the liquid must be equal to the rate of mass transfer of liquid vapor (e.g. water vapor) to the gas multiplied by the enthalpy of vaporization per unit mass of the evaporating component. The water temperature will adjust until the local rates of heat and mass transfer are balanced. Note that the vapor concentration of the evaporating component at the interface will generally be at the vapor pressure of the liquid, and that this is a strong function of temperature. This is sometimes referred to as the wet bulb temperature since the same principle is used to determine relative humidity in air by comparing the temperatures of a dry bulb thermometer with a wet bulb thermometer. This mechanism can lead to a large amount of water vapor in the discharge particular when the discharge is in direct contact with the water surface and a large power flux is incident onto the liquid as is the case for a DC glow discharge with a liquid cathode [324].

\section{Reaction rates in gas and liquid phase}

\subsection{Introduction}

The plasma science and engineering community needs the assistance of researchers in many other areas to provide the fundamental data required to understand and exploit the phenomena we observe in plasma-liquid interactions. To make full use of the currently available data and to identify new data which are most critically needed from other areas (including fundamental experimental and theoretical atomic and molecular collision physics, radiation physics, and gas and liquid phase chemistry) the plasma community needs to define the specific environments and species of interest. In the case of plasmas and liquids, these environments are basically the initiating plasma, the plasma-liquid interface (including immediately above and below the interface) and the bulk liquid.

For example, in the case of atmospheric plasma jets one may need to consider (a) an inert gas initiating a plasma, which interacts with the gas phase of increasing humidity as a water or other liquid surface is approached, (b) the impact of charged and neutral particles and photons and penetration or migration of these species through the water surface, (c) the neutral and ion chemistry of short and long lived species immediately below the surface and (d) the resultant bulk liquid chemistry. In each of these environments the user community should estimate typical time scales, major expected species and define the dominant collision regimes (e.g. ionneutral, neutral-neutral, radical-radical or radical-neutral, solvation or clustering) to help guide the production of data.

The data that is required are now distributed over a vast range of literature. Here we attempt to provide the reader with starting points for data searches. A few examples of published work where large reaction and chemical kinetics model data sets for gas phase plasmas include: helium based, water containing atmospheric pressure plasmas [325-330], argon based systems [331], for neon-xenon based systems [332] and humid air [57, 329, 333]. Recently there have been major developments in modeling activities to include the interaction of helium plasmas into air impacting on liquid water and the resultant chemistry within the liquid which contain extensive references to data for the gas, liquid and gas-liquid interface [62, 188]. All data sets should be used with caution. However, the recent success of models predicting specific experimental measurements, (for example, [334-337]) can provide additional confidence in the quality of the available data.

\subsection{Gas phase}

6.2.1. Electron molecule scattering. Comprehensive reviews of the data from binary collisions of electrons with $\mathrm{H}_{2} \mathrm{O}$, both 
experimental and theoretical are available [338-342]. A typical test of the applicability of data sets of cross sections for electron and ion transport is whether the fundamental datasets can be used to reproduce experimental swarm measurements by using the datasets in solutions of Boltzmann's equation for the velocity distributions. The swarm data are typically Townsend ionization coefficient $\alpha$, drift velocity $w$, mobility $\mu$, diffusion coefficient $D$, and characteristic energy $e D / \mu$ derived from measurements of electrons or ions drifting in constant values of $E / N$ (electric field/gas number densities). Often the initially generated data set of fundamental cross sections does not reproduce experimental swarm data due to there being missing data (e.g. vibrational excitation cross sections) or small inaccuracies to which the derived transport coefficients are very sensitive. It is then typical practice to make small adjustments to the datasets so that the experimental swarm data can reproduced. This then places high importance on the accuracy and availability of experimental swarm data. In addition to the older measured transport data [343-345], newer measurements have been made [346, 347]. There are several sets of cross sections that were derived based on the swarm (transport) technique [342, 348-352] which are based on several measurements of swarm data [18, 347, 351, 353-355]. While electron-molecule data are available for water vapor, mostly derived at low pressure; at atmospheric and higher pressures it may be necessary to include additional complex three body processes [19].

Most of these data sets are derived from the low energy transport data $(1-2 \mathrm{eV})$ that do not overlap very well with the ionization cross section, even with the mean energies of electrons found in gas discharges. For that region, the swarm derived sets of transport coefficients often make extrapolations based on the available binary collision data. Results of de Urquijo [20, 347] that include multiplication (ionization minus attachment) coefficients have helped to extend the energy range. Further extensions of ionization coefficients are needed using swarm measurements at high $E / N$ [356]. Swarm analysis at somewhat higher energies (above $5 \mathrm{eV}$ ) rely heavily on ionization cross-sections being accurate [357] and those for water are covered reasonably well [358-360].

In the $E / N$ range from $30 \mathrm{Td}$ to $90 \mathrm{Td}\left(1 \mathrm{Td}=10^{-17} \mathrm{~V} \mathrm{~cm}^{2}\right)$, electrons are strongly influenced by the rapidly falling elastic and rotational cross sections (energies between 0.04 and $2 \mathrm{eV}$ ) $[350,351]$ of water which results in a rapid increase of all transport coefficients and mean electron energy. Under these conditions, the hydrodynamic approximation in analyzing swarm data may not be valid and a non-hydrodynamic kinetic (nonlocal) model may be required to both calculate and analyze the experimental data, including anisotropic scattering. Testing and rescaling of the available cross sections using non-hydrodynamic techniques may provide more accurate results and comparisons with experimental data that extend to high $E / N$.

Data also exist for water vapor mixtures, most importantly humid air [361] and mixtures with He and Ar [347]. Experiments have shown that attachment to water vapor does not occur at thermal energies [362] so observations of $\mathrm{H}_{2} \mathrm{O}^{-}$ions at low $E / N$ may be due to hydrated electrons being formed. Data for electrons colliding with the relevant reactive species $\left(\mathrm{OH}\right.$ and $\left.\mathrm{H}_{2} \mathrm{O}_{2}\right)$, and water molecule clusters are mostly missing but some of the required data is beginning to emerge.

6.2.2. Ions in water vapor. The key challenge in measuring swarm parameters for ions in water vapor is the means of ion formation. In the case of ions that are produced in gases or liquids one has to reconstruct the entire multistep kinetics that may form a variety of ions through charge exchange to deconvolve the swarm data. In doing so, the transit time is difficult to establish because the identity of ions may change due to ion-molecule reactions en route from the anode to the cathode. Ions are good nucleation centers for the formation of clusters and their size may vary with distance and conditions which may then result in swarm measurements being pressure dependent and not dependent only on E/N. Experiments with a variable drift length may be used to perform comparative measurements to decipher these dependencies. These issues require advanced theoretical and numerical treatments even in simplified swarm systems to deconvolve the raw swarm data.

The usual practice in the ion swarm community is to provide interaction potentials that can be used to determine differential cross sections. Most models relevant to plasma-liquid interactions typically require total cross sections with simplified models for angular distributions (e.g. forward scattering, isotropic or backward scattering). Transport coefficients can then be determined from the cross-sections and both can be applied in models [363-366].

Transport data for ions in pure water (vapor) exist for a few ions originating from $\mathrm{H}_{2} \mathrm{O}$ in a limited energy $(E / N)$ range: $\mathrm{H}_{2} \mathrm{O}^{+}, \mathrm{OH}^{+}, \mathrm{O}^{+}, \mathrm{O}_{2}^{+}, \mathrm{H}_{3} \mathrm{O}^{+}$and $\mathrm{H}^{+} . \mathrm{H}^{+}$is particularly important as it is the lightest and thus the fastest ion, and it also leads to production of fast $\mathrm{H}$ atoms. Cross sections for fast neutrals may be required to explain the high rates of excitation for large values of $E / N$ [367] and in the cathode fall. A collection of cross sections for scattering of fast $\mathrm{H}$ atoms on $\mathrm{H}_{2} \mathrm{O}$ can be found in [368], which comprises available experimental data with cross sections obtained by scaling from corresponding electron impact cross sections.

The most prominent negative ions directly produced from water vapor are $\mathrm{O}^{-}$and $\mathrm{OH}^{-}$, although other negative ions such as $\mathrm{H}^{-}$and $\mathrm{H}_{2} \mathrm{O}^{-}$also occur in small quantities [369]. Cross section and transport sets are being developed for other ions as well [370]. Recent measurements of transport data for negative ions [371] in water vapor revealed that clusters are formed and that cluster size depends on pressure and other conditions $[372,373]$. It is important to complete the cross section set for water-based clusters and to develop accurate models of cluster formation and related transport properties. Those data could be developed by implementing techniques currently used to study general hydration of charged particles.

Data for water related ions, both positive and negative, are abundant for humid noble gasses [374, 375] and are available for humid air [376]. General sources of data for ion transport coefficients can be found in $[366,377,378]$ and in the LXCat database [349]. These data enable calculations of the transport 
coefficients for relevant ions in some of the gases found in discharges in proximity to liquids.

A wide variety of ion-molecule reactions occur in the gas phase containing water vapor [379-386] including detachment reactions that have a number of different channels and a strong energy dependence [387-389]. New data or improvements to existing data are needed. However, including every possible reaction is often not practical. A strategy is needed to establish a reliable database for the primary reactions that can be extended to more complex systems adjacent to the liquid surface where the gas may be super-saturated with water vapor and intersects with the ambient gases. This is a region where there are significant gradients in the water content and also in temperature. While there is gas phase data available for ion-molecule reactions for most of the individual constituents in this region [386, 390], the accuracy of binary gas phase rate coefficients in this environment is questionable due to the propensity for forming water cluster ions. Descriptions of clustering, hydration and release from the hydrated state can be based on either simpler phenomenological models (effective lifetimes) or on more detailed kinetics. Models show that there are significant populations of $\mathrm{H}_{3} \mathrm{O}\left(\mathrm{H}_{2} \mathrm{O}\right)_{n}^{+}$, up to $n=8, \mathrm{NO}\left(\mathrm{H}_{2} \mathrm{O}\right)_{n}^{+}$, up to $n=3$ or 4 for positive ions and $\mathrm{O}\left(\mathrm{H}_{2} \mathrm{O}\right)_{n}^{-}, \mathrm{OH}\left(\mathrm{H}_{2} \mathrm{O}\right)_{n}^{-}, \mathrm{NO}\left(\mathrm{H}_{2} \mathrm{O}\right)_{n}^{-}$up to $n=3$ or 4 for negative ions. $\mathrm{CO}$ and $\mathrm{CO}_{2}$-based negative ion clusters also need to be considered.

6.2.3. Neutral chemical reactions. Data for neutral-neutral chemical reactions relevant to plasma contacting liquids are typically scattered over a number of sources. The most arduous work involved in the modeling of chemical kinetics, and of plasma chemistry in particular, is the collection of reaction rate coefficients to formulate reaction mechanisms. In addition to electron impact processes, those mechanisms include reactions between neutral atoms and molecules, interactions with radicals, ion-molecule chemistry and reactions with or at surfaces. The interest in reaction mechanisms for atmospheric plasmas interacting with liquids was preceded by modeling and experiments on the use of atmospheric pressure plasmas for remediation of toxic gases from air. These earlier chemical reaction sets of humid air were compiled and developed by the group of Kushner [325] and many other groups working on corona treatment of air [329]. More recently a chemical reaction set describing $\mathrm{He}-\mathrm{O}_{2}-\mathrm{H}_{2} \mathrm{O}$ plasma chemistries has been developed with reaction rate coefficients collected from different fields (44 species, 577 reactions), described in [327]. Additions and modifications have recently been made to this reaction mechanism and it has been validated for a diffuse $\mathrm{RF} \mathrm{He}-\mathrm{H}_{2} \mathrm{O}$ glow discharge by comparing predicted densities for ${ }^{\bullet} \mathrm{OH}$ and $\mathrm{H}_{2} \mathrm{O}_{2}$ with experiments [336]. Earlier work on helium discharges with humid air impurities was reported in [326] and recently extended in [330]. A large chemical reaction set of Ar discharges containing humid air is reported in [331]. This reaction mechanism was validated by comparing predictions for $\mathrm{O}_{3},{ }^{\bullet} \mathrm{NO}$ and $\mathrm{O}^{\bullet}$ densities from a plug flow simulation with measurements made in a cold atmospheric pressure plasma jet $[334,337]$. These reaction mechanisms have met with success in many venues. For example, humid air chemical kinetics models have been used to explain key reaction pathways in water vapor containing discharges [57, 333, 391].

Most of the plasma chemistry models developed for discharges in contact with liquids and generally in humid atmosphere rely on extensive compilations of data. Most notable are the evaluations of gas phase chemical reactions of neutral species involved in atmosphere chemistry [392, 393], databases for positive ion-molecule reactions [386] and neutral-neutral chemical reactions in non-thermal humid air plasmas [394] and for dry air chemistry [395].

Further sources of data can be found in the combustion community. Combustion and plasma processes have similarities in their approaches to experiments and modeling. For example, data used in cool flames and combustion simulations are directly applicable to modeling low temperature plasmas [396]. There are also large differences between plasma and combustion chemistry. For example, high temperature combustion, which is often cited as a source for rate constants in plasma processes, rely on different mechanisms compared to low temperature plasma processes and should therefore be used with care and thoroughly assessed before use. One important aspect of combustion modeling is the hierarchy that has been developed for validation of the simulation. This validation can be accomplished by many techniques, including significance analysis [397] and pathway analysis [398]. A similar validation hierarchy is being developed for low temperature plasma modelling [399]. The combustion community has gained advantage from using standardized reaction mechanisms such as GRIMech, which is essentially a list of community endorsed elementary chemical reactions and associated rate constants, a practice that would also benefit the low temperature plasma community [400, 401].

While data for the ground-state neutral reactions are relatively well represented in the literature, quantitative data for excited-state reactions is much less extensive. The interpretation of optical emission spectroscopy (OES) requires knowledge of production and quenching rates of the excited species. For example, in addition to electron-impact excitation, electron-ion dissociative recombination is an important channel in the production of excited species [402]. The data for these processes can be found in databases originally developed for lighting, upper atmospheric chemistry and excimer lasers [403, 404]. Reliable data sets are also needed for collisional quenching, three body quenching, rotational energy transfer (RET), and vibrational energy transfer (VET). Such data are critical for diagnostic techniques such as laser induced fluorescence (LIF). Data on the temperature and pressure dependencies of these processes is limited and most data is for the lowest excited states. There are several compilations that include data for excited states of ${ }^{\bullet} \mathrm{OH}, \mathrm{O}^{\bullet}, \mathrm{O}_{2}, \mathrm{~N}^{\bullet}, \mathrm{N}_{2}$. For example, Herron and Green proposed a reaction grid for neutral species in air plasmas and critically evaluated available data [394], while Schofield evaluated rate constants and their temperature dependences for reactions that involve metastable states of atomic oxygen $\left(\mathrm{O}\left(2^{1} \mathrm{D}_{2}\right)\right.$ and $\left.\mathrm{O}\left(2^{1} \mathrm{~S}_{0}\right)\right)$ [405]. The same author reviewed available gas phase chemical kinetic 
rate constants for the interactions of the low lying electronic states of several atoms and molecules, including $\mathrm{N}\left(2^{2} D_{3 / 2,5 / 2}\right.$, $\left.2^{2} P_{1 / 2,3 / 2}\right), \quad \mathrm{O}_{2}\left(c^{1} \sum_{\mathrm{u}}^{-}, C^{3} \Delta_{\mathrm{u}}, A^{3} \sum_{\mathrm{u}}^{\mp}, B^{3} \sum_{\mathrm{u}}^{-}\right), \quad \mathrm{OH}\left(A^{2} \sum^{+}\right)$, with several collision partners [406]. Golde reviewed reactions and production of $\mathrm{N}_{2}\left(A^{3} \sum_{\mathrm{u}}^{\mp}\right)$ [407]. Reactions of the first two electronically excited states of atomic nitrogen: $\mathrm{N}\left({ }^{2} D\right)$ and $\mathrm{N}\left({ }^{2} P\right)$, and of the first excited state of molecular nitrogen $\mathrm{N}_{2}\left(A^{3} \sum_{\mathrm{u}}^{\mp}\right)$ are evaluated in [408]. Papers from the combustion community offer some data on collisional quenching, VET [409] and RET coefficients [410] for ${ }^{\bullet} \mathrm{OH}$. A source of collisional quenching rate constants for metastable states of rare gases is [411]. Several books are also a valuable source of data [43, 412-414].

Overall, there are many good starting points for developing kinetic models of chemistry in water vapor containing plasmas, at least at room temperature while there are uncertainties at elevated temperatures and at non-standard pressures. There are also uncertainties in roles of fast neutrals and vibrational excited species in reaction mechanisms. Data addressing the generation and disposition of molecular and atomic clusters are needed to model the highly humid near-surface regions. Tests of the validity of data or critical evaluations for particular pathways are continuously needed including comparison with experiments. At the Lorentz Center meeting that initiated this review, a question was raised on the required reliability of experimental data for these comparisons. The attendee generally agreed that, at this stage in the field, a difference between experimental measurements and model predictions of up to factor of 2-5 is often acceptable for complex plasma chemistries.

\subsection{Bulk liquid}

The development and modeling of reaction mechanisms in the liquid phase require rate coefficients in the same manner as in the gas phase. Gas phase reactions typically involve collisions between two, and at very high pressures perhaps three species, in discrete separable events. Reactions in liquids are continuously perturbed by the presence of the solvent. Due to the discrete nature of these reactions in gases, rate coefficients for use in gas phase mechanisms can be determined by gas kinetic theory, molecular dynamics, and/or quantum chemical methods. However, due to the multi-body nature of reactions in liquids, there is no unified approach for theoretically deriving reaction rate coefficients in liquids [415]. Species densities in liquids can often be derived based on thermodynamic equilibrium. However, near plasma-liquid surfaces, reactions can be dominated by the non-equilibrium reactants that enter the liquid from the plasma. In these cases, a non-equilibrium, rate coefficient based analysis is required. For a detailed discussion of available data in the interfacial gas-liquid region, see sections $3-5$.

A major source of relevant rates and identification of chemical mechanisms and pathways in the bulk liquid can be found in textbooks, journal articles and data bases of the radiation and sonochemistry communities. Some examples of textbooks are [78, 201, 416-418]. Two recent publications on plasma-liquid interactions which contain more pertinent and up to date data are [62, 188], while accurate thermophysical properties for a number of liquids, including water, are available at NIST Standard Reference Data website [419].

The AOP community also offers a rich source of basic data. This data typically focuses on ${ }^{\bullet} \mathrm{OH}$ reactions since they play an important or even dominant role in AOP due to the extreme reactivity and non-selectivity of ${ }^{\bullet} \mathrm{OH}$ which can be formed by a number of different mechanisms. Overviews of rate constants used by the AOP community are available [184, 242]. These datasets have been developed using various approaches. The group contribution method (GCM) used to predict ${ }^{\bullet} \mathrm{OH}$ constants in the gas phase [420-422] has emerged as the most accepted method and is implemented in U.S. Environmental Protection Agency software [423]. However, applying these methods to the liquid phase requires considering changes in functional group properties arising from the close spaced intra-molecular environment, for example, electronic push-pull effects, intermolecular hydrogen bond formation and steric effects. Recently, linear free energy relationships (LFER) have been developed to address some of these challenges [424, 425]. In addition a GCM for aqueous phase $\mathrm{OH}$ radical constants has been developed [163]. Other molecules in the aqueous phase may act as promoters, capable of regenerating the superoxide anion, or as inhibitors of radical reactions, and these species have strong influences on the chemistry. There have been many studies of such effects but generally this work focuses on reactions of biological molecules. Data is also needed on the dependence of reaction rates on $\mathrm{pH}$ and temperature.

Studies of electron transport in liquids have been carried out for many years up to the present. White et al [426] in generalized studies of soft condensed matter have included structure factors to account for multiple scattering in the high pressure liquid phase [427]. More recently this work has been extended to water and other liquids [426, 428]. The conclusion is that for energies above $5 \mathrm{eV}$ one may be able to employ a binary collision approximation. At lower energies one needs to define different cross sections for calculation of momentum transfer and of energy loss. This approach, however, does not take into account more serious perturbations to the transport such as hydration.

Further work on how electrons can gain and dissipate energy in liquid water under influence of electric fields is necessary to establish the breakdown mechanism. More data is needed on the role of hydrated electrons and their propagation in the bulk liquid and the surface.

\section{Diagnostics}

\subsection{Introduction}

In view of the complexity of plasmas in and in contact with liquids, diagnostics are of key importance to increase our understanding of these plasmas. This section reviews the stateof-the-art in diagnostics and identifies important challenges. 


\subsection{Gas phase diagnostics}

Gas phase diagnostics have been developed to measure plasma parameters and densities of species present in the plasma, and so improve our understanding of their operation. Diagnostics that are typically used in low pressure plasmas, such as OES, optical absorption spectroscopy, LIF, actinometry, mass spectroscopy and electrical probe measurements are complicated by collisional processes when applied to the atmospheric pressure plasmas of interest to plasma-liquid interactions. From a practical matter, in many cases, these diagnostics cannot be used at atmospheric pressure. In other cases, such as OES and LIF, complex modeling is required to account for collisional processes to properly interpret the data obtained at atmospheric pressure [429-431]. Similar laser based diagnostics are used in combustion research and techniques are available from that community for interpreting LIF [431, 432].

Two recent papers review the use of diagnostics in atmospheric pressure plasmas [433, 434]. An overview of diagnostics relevant to measuring plasma parameters and species densities in atmospheric pressure plasmas is in table A1 in the appendix. The majority of these techniques are optical diagnostics which require stable and reproducible plasmas as signal-to-noise ratios are often improved by the accumulation of data from several measurements. Line-of-sight diagnostics such as absorption spectroscopy [334, 435] require some tomographic technique, such as an Abel inversion, to produce spatially resolved data. However, even methods such as LIF, where direct imaging produces spatially dependent data, may suffer from degraded resolution by time averaging if plasmas are not reproducible in space or time. This loss of resolution is an issue for turbulent plasma jets and when the gas flow has a strong influence on the position of the plasma filament. In fact, plasma jets that appear laminar and well behaved in a time averaged image have shown nearly chaotic behavior with single shot imaging [302]. Similarly, deformation of the plasma-liquid interface can lead to random formation of plasma filaments [291] which also require single shot laser measurements to properly resolve. This approach, including saturated LIF, has a long history in diagnostics applied to turbulent combustion but has not been used extensively in the field of plasmas [436].

If spatial resolution is not required several techniques can be used to correlate plasma produced species with reactivity produced in the liquid phase. Such approaches include measuring gas phase densities with mass spectrometry at an interface [437], measuring the terminal long lived products using a multi-pass cell for Fourier transform infrared (FTIR) absorption spectroscopy [438] or quantum cascade laser absorption spectroscopy [439]. These data may provide good assessments of densities for remote plasma jets which do not directly interact with the liquid. However, care should be taken when extrapolating these results to plasma jets that directly interact with the liquid. The effects of changing flow patterns or increasing water vapor concentration may significantly impact the long-lived species even in the effluent of the jet. For example, Schröder et al directed the effluent of an APPJ onto different solid surfaces and showed that the distribution
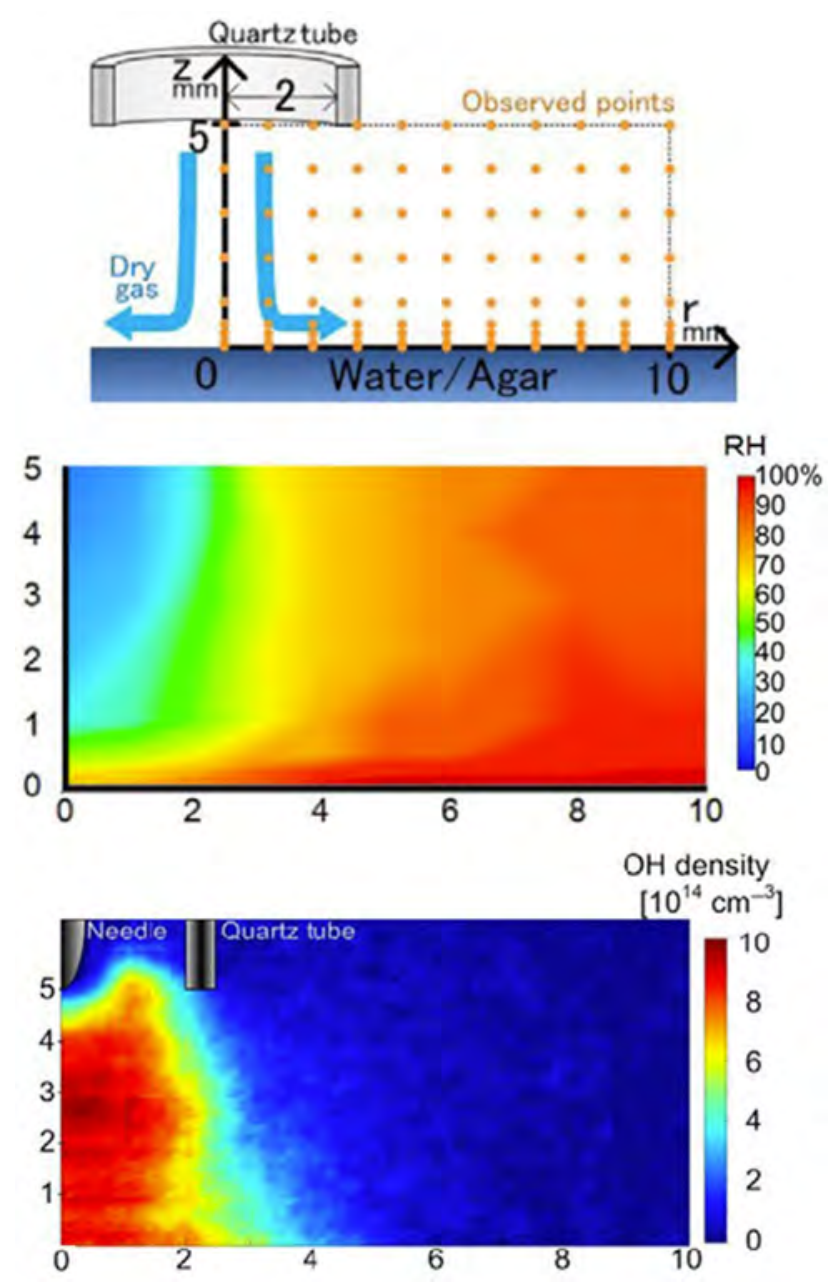

Figure 19. Example of water concentration and ${ }^{\bullet} \mathrm{OH}$ in a cold atmospheric pressure He plasma jet impinging on a water surface. The water concentration is obtained through fluorescence lifetime measurements of the jet seeded with ${ }^{\bullet} \mathrm{NO}$ and the ${ }^{\bullet} \mathrm{OH}$ density is measured by ${ }^{\bullet} \mathrm{OH}$ LIF. Reproduced with permission from [445], copyright 2015 IOP Publishing.

of atomic oxygen, and reactive oxygen species (ROS) in general, depends on the character of the surface [440]. These measurements were made by two- photon absorption laser induced fluorescence (TaLIF) measurements.

Species are transferred from the liquid to the gas phase due to evaporation and sputtering when plasmas interact with liquids [24]. Water is electronegative and undergoes rapid vibrational relaxation that can lead to significant gas heating [441]. Since water is also a strong quencher of excited molecules such as $\mathrm{OH}(A)$ [442] the spatial dependence of quenching rates needs to be accurately known to properly derive ${ }^{\circ} \mathrm{OH}$ densities from LIF measurements near the liquid interface. An example of an LIF measurement of ${ }^{\bullet} \mathrm{OH}$ density in a plasma jet impinging on water is in figure 19. Similarly, Ries et al [443] and Yonemori et al [444] measured the ${ }^{\bullet} \mathrm{OH}$ distribution near dry and (partially) humid surfaces using LIF, and showed that with increasing humidity of the surface, the ${ }^{\bullet} \mathrm{OH}$ concentration directly above the surface also increased.

TaLIF of atomic $\mathrm{O}^{\bullet}$ and $\mathrm{H}^{\bullet}$ have quenching times shorter than $1 \mathrm{~ns}$ for many relevant conditions at atmospheric pressure 


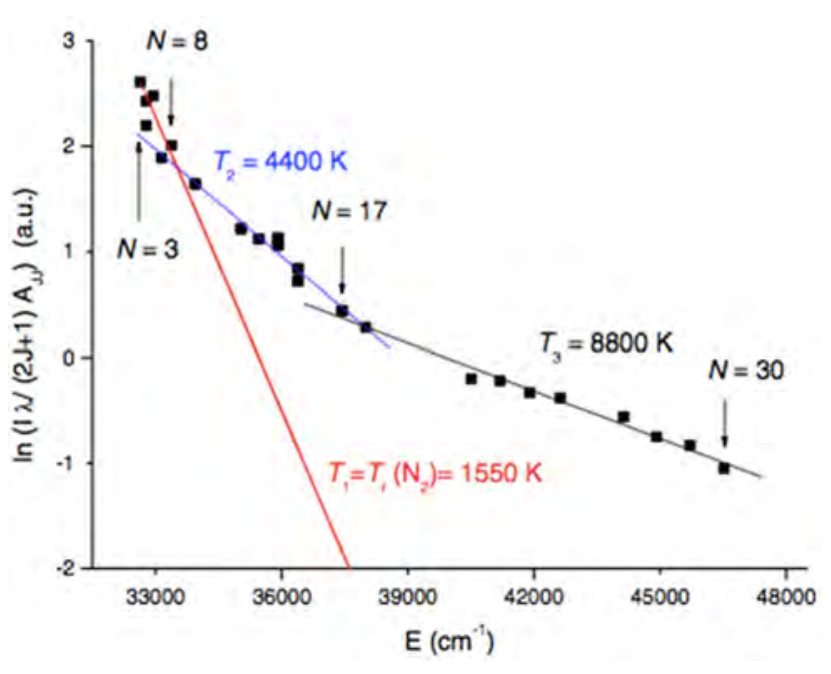

Figure 20. Boltzmann plot of the rotational distribution of $\mathrm{OH}(A)$ obtained from the emission spectrum of $\mathrm{OH}(A-X)$ from a discharge in a vapor bubble in water. Reproduced with permission from [455], copyright 2014 IOP Publishing.

and these measurements are challenging using nanosecond pulsed lasers [446]. New laser and detection systems with sub-nanosecond pulses and corresponding sub-nanosecond temporal resolution enable measuring the effective lifetimes of excited states required for absolute density measurements [447, 448].

Due to the often limited accessibility of active (laser) diagnostics and the complicated reactor geometries involving liquids, OES is the most common diagnostic used to investigate plasmas in liquids. The emission spectra of such plasmas are often dominated by the $\mathrm{OH}(A-X)$ ro-vibrational band and the hydrogen Balmer lines [449]. These features provide a means for obtaining gas temperatures and electron densities. However, in general the production processes of excited species and the subsequent plasma kinetics strongly influence the OES spectra, and in turn complicate the interpretation of the data. OES based studies of liquid plasmas use combinations of absolute OES and modelling to yield species densities $[48,450]$. To our knowledge, one result has been reported on - $\mathrm{OH}$ density measurements in a submerged arc discharge by absorption spectroscopy [451]. In all cases, the dynamics of the liquid interface may influence the path of the beam and so affect the spatial resolution of the measurement.

Researchers often use $\mathrm{OH}(A-X)$ emission as a measure of the density of $\mathrm{OH}(X)$. However, $\mathrm{OH}(A)$ can be produced in many ways [452] and many assumptions need to be made to derive the density of $\mathrm{OH}(X)$ from the luminescence of $\mathrm{OH}(A)$. Typically the $\mathrm{OH}(A)$ emission intensity decreases with increasing $\mathrm{H}_{2} \mathrm{O}$ concentration [453] while the $\mathrm{OH}(X)$ density tends to increase with increasing $\mathrm{H}_{2} \mathrm{O}$ concentration [454].

Even in highly collisional plasmas, there may be strong deviations from equilibrium rotational distributions. As described in [455] the rotational distribution of $\mathrm{OH}(A)$ is often non-Boltzmann (see figure 20). For many conditions occurring in atmospheric pressure plasmas in the presence of liquids, the characteristic collisional quenching time of $\mathrm{OH}(A)$ by $\mathrm{H}_{2} \mathrm{O}$ is of the same order as the rotational energy transfer
Table 2. Reaction rates of L-histidine with some major components produced by the plasma [230].

\begin{tabular}{ll}
\hline Reacting species & Reaction rate $\left(\mathrm{M}^{-1} \mathrm{~s}^{-1}\right)$ \\
\hline${ }^{1} \mathrm{O}_{2}^{*}$ & $3.2-9 \times 10^{7}$ \\
$e_{(\mathrm{aq})}^{-}$ & $6 \times 10^{7}$ \\
$\bullet^{\circ} \mathrm{OH}$ & $4.8 \times 10^{9}$ \\
$\mathrm{O}_{3}$ & $3.9 \times 10^{3}$ \\
$\mathrm{O}_{2}^{\bullet-}$ & $<1$ \\
$\mathrm{H}^{\bullet}$ & $2.3 \times 10^{8}$ \\
$\mathrm{HO}_{2}$ & $<2.3 \times 10^{8}$ \\
\hline
\end{tabular}

Note: The reaction rate constants can be $\mathrm{pH}$ dependent.

time (rotational relaxation). Under these conditions the $\mathrm{OH}(A)$ distribution of rotational population does not thermalize. As a result, the distribution is strongly influenced by the formation process that produces the initial distribution and which is often a non-Boltzmann distribution.

Atomic excitation temperatures are also often measured by OES. However, atomic excitation temperatures are not necessarily the same as electron temperatures. This equality only occurs in plasmas in LTE. By assuming LTE, atomic emission spectra in sonoluminesence can be successfully produced, although deviations from equilibrium have also been found [456-458]. The excitation temperature can depend on the energies of the emitting atomic levels and the production mechanism of the excited states. Collisionalradiative models are usually necessary in non-equilibrium plasmas to fully interpret experimentally measured line intensity ratios.

Continuum radiation can be the dominant emission from high density direct discharges in liquids [459]. In high electron density plasmas continuum radiation mostly originates from free-free bremsstrahlung and from free-bound recombination between electrons and ions [460]. In plasmas with a low ionization degree, continuum radiation emitted through free-free interactions between electrons and neutral atoms becomes important. In the presence of hydrogen or water vapor there may be a significant contribution of $\mathrm{H}_{2}$ radiative dissociation continuum in the UV [461], which is most likely the case for plasmas in bubbles. In many cases, the continuum radiation in plasmas and liquids has been attributed to black body radiation originating from the electrode [462]. There has been much discussion in the literature on the origin of continuum radiation in sonoluminescence which includes chemoluminescence processes [456, 462-464]. However, this emission is highly dependent on the precise plasma conditions. Most plasmas of interest are optically thin, although exceptions include sonoluminescence [464] and exploding wires discharges in water [464].

While discharges in the gas phase interacting with liquids can be produced in DC mode, the majority of discharges in liquids and bubbles are highly transient in nature. These conditions reach their limit in sub-nanosecond pulsed direct discharges in liquids and single bubble sonoluminescence. As mentioned earlier, the sonochemical plasma formed inside the bubble as it collapses has a diameter of less than $1 \mu \mathrm{m}$ [466], 
and its lifetime is $\approx 100$ ps [120]. Time resolved optical diagnostics in direct discharges in liquids often reach the detection limit in both space and time in resolving the emission on a relevant time scale of plasma dynamics [23]. As these discharges are often not reproducible in space, spatially resolved measurements are difficult. A unique spatially resolved electron density measurement in a streamer channel in liquid water has been reported by An et al [21] which shows a significant dependence of the electron density on the position within the plasma filament.

Discharges both in small bubbles and directly in water can produce significant pressure gradients on nanosecond time scales. Mach-Zehnder interferometry has been used to quantify these transients in secondary streamers in liquids [21]. To computationally reproduce experimental data, short 2-3 ns pressure pulses with amplitudes of 2-3 GPa were required [21], as confirmed using Schlieren imaging to measure bubble expansion [467]. Similar effects have been investigated for nanosecond pulsed spark discharges in atmospheric pressure air by Laux et al [468].

There are exceptional challenges in using diagnostics for multiphase plasmas consisting of sprays and droplets. These conditions may include the formation of aerosols and droplets by instabilities of the liquid interface such as Taylor cones. The challenges lay in the randomness, size of aerosols below or in the range of the optical diffraction limit and the corresponding potential spatial gradients of plasma properties on similar length scales. Investigations of multiphase combustion processes have been performed [469-471] and may serve as a guidance to explore similar techniques within the plasma community.

\subsection{Liquid phase diagnostics}

Short and long-lived chemical species are produced in the liquid by plasmas either directly or transferred from the gas phase plasma being in contact with the liquid. These species can react at or penetrate through the plasma-gas/liquid interface and dissolve into the bulk liquid, and can initiate secondary chemical processes. Due to the dense hierarchy of the reactions giving rise to both stable and non-stable intermediates and reactions products, analysis of aqueous chemistry in plasma-treated liquids is often complex.

The level of detail required in analysis and measurement of liquid phase species often depends on the application. For example in biology, the activity of reactive oxygen and nitrogen species (RONS) in vivo might not directly correlate to specific and individual compounds but to a general redox activity, and so is expressed by the concept of oxidative stress [472] or redox-based wound healing [473, 474]. For these conditions, it suffices to measure the redox activity that is generated in liquids by plasma treatment as opposed to measuring specific species densities. One such method is measuring the redox potential in the liquid after plasma treatment using wellestablished standard electrode systems (e.g. standard hydrogen electrode (SHE), standard calomel electrode (SCE), standard $\mathrm{Ag} / \mathrm{AgCl}$ electrode) [475]. Another possibility is using redox indicators such as ABTS (2,2'-azino-bis(3-ethylbenzthiazoline-6-sulphonic acid)) that generate a stable green-colored radical cation detectable at $405 \mathrm{~nm}$ in an oxidative aqueous environment [476].

On the other hand, fundamental understanding of plasma induced liquid phase processes will require detailed knowledge of the plasma induced reactive chemistry in liquids. In the presence of air both ROS and reactive nitrogen species (RNS) are produced. Relatively small changes in plasma conditions often lead to large relative variations in production of RNS and ROS which could shift chemical reaction pathways in liquids. As a result, there is a great interest in measuring transient or short-lived reactive species and radicals produced directly and indirectly by plasma in liquids such as ${ }^{\bullet} \mathrm{OH}, \mathrm{O}_{2}^{--},{ }^{\bullet} \mathrm{NO},{ }^{\bullet} \mathrm{NO}_{2}$, peroxynitrite $\left(\mathrm{ONOO}^{-}\right)$and $\mathrm{O}_{3}$. Some of these species are difficult to measure due to their short lifetimes and fast disproportionation in plasma/ liquid systems. While many short-lived species have known absorption spectra [477], absorption measurements are challenging for plasma produced species as their densities are often low. The absorption spectra in liquids are also broad and often overlapping, which leads to challenges in the deconvolution of the absorption spectra of complex mixtures of reactive species. The end result is that measurements of plasma produced reactivity in liquids are most often of longlived species such as hydrogen peroxide, nitrites, nitrates, molecular hydrogen and oxygen.

The measurements of reactivity in plasmas should be made with careful consideration of the organic content of the liquid. For example, ${ }^{\bullet} \mathrm{OH}$ are produced at the interface of most liquids in contact with plasmas. A very rapid process is the reaction of two ${ }^{\bullet} \mathrm{OH}$ radicals forming $\mathrm{H}_{2} \mathrm{O}_{2}$ which actually reduces the overall reactivity of the liquid. If there are organics in the liquid, reaction of ${ }^{\bullet} \mathrm{OH}$ with the organic can sustain reactivity by forming a new radical, and further reactions will be triggered. This sequence can lead to transfer of reactive species directly induced by the plasma to radicals which strongly depend on the organic molecules present in the liquid. A well-known example is the peroxidation of lipids. The reaction of ${ }^{\bullet} \mathrm{OH}$ with the lipids produce lipid peroxyl radicals and lipid peroxide [478].

Measuring reactive species in liquids could also be used to assess plasma reactivity. In many applications involving plasma and liquids, it is desirable not only to know the density of plasma constituents but also the actual flux of reactive species from the plasma reaching the liquid. While gas phase measurements can provide valuable insights, it is not straightforward to determine the flux reaching the target liquid from these measurements due to the complex transport processes encountered at the gas-liquid interphase. Measurement of the density of a long lived species such as $\mathrm{H}_{2} \mathrm{O}_{2}$ in the liquid may in some cases correlate well with the fluence of $\mathrm{H}_{2} \mathrm{O}_{2}$ from the gas phase. This approach motivates the use of plasma dose to characterize reactivity. However, one should carefully use the term dose, a term that could have different meanings and not be comparable between different plasma sources. Oxidation potential of the liquid might also be seen as a plasma dose concept. Whatever 
the definition of plasma dose, it remains a generalization and it does not express which species are responsible for the oxidation. This is particularly the case as different mixtures of species could lead to the same oxidation potential.

Reaction pathways can be investigated non-quantitatively by adding specific scavengers to solutions, a common method in biological investigations. This technique blocks certain chemical pathways and is therefore useful to check reaction mechanisms. Examples of scavengers include superoxide dismutase (SOD) for $\mathrm{O}_{2}^{--}$, catalase of $\mathrm{H}_{2} \mathrm{O}_{2}$, D-mannitol for ${ }^{\bullet} \mathrm{OH}$ and carboxy-PTIO for ${ }^{\bullet} \mathrm{NO}$ [230, 479-481]. However, scavengers can be non-selective; particularly scavengers based on non-enzymatic reactions. An example of non-selective scavenging for L-histidine which is often used as a scavenger of singlet-delta oxygen is in table 2 . In addition, chemicals used as scavengers could also be modified by the plasma treatment. Therefore, it is necessary to keep the number of chemicals and reaction partners as low as possible to prevent misleading artifacts [482, 483]. For the complex chemically active mixtures created by plasma treatment, studies using scavengers should always be combined with positive controls.

Various types of analytical methods with different selectivity, sensitivity and precision are used in the investigation of plasma-treated aqueous liquids. These techniques include semi-quantitative methods using test paper strips which are dipped into the plasma-treated water to measure, for example, $\mathrm{H}_{2} \mathrm{O}_{2}, \mathrm{NO}_{2}^{-}, \mathrm{NO}_{3}^{-}$. Colorimetric measurements utilizing spectrophotometric assays are based on measuring the intensity of a color complex formed by reaction of the particular species with the relatively specific colorimetric reagent. These methods can be quantitative. An example is the yellow colored titanyl sulfate complex formed when titanium sulfate reacts with $\mathrm{H}_{2} \mathrm{O}_{2}$ [484]. This technique can be complemented by the bleaching of blue colored indigo dye by $\mathrm{O}_{3}$ [485] or pink colored azo dye formed by Griess diazotization reactions with $\mathrm{NO}_{2}^{-}$[486]. A more sensitive, though not always fully specific, technique is based on fluorescence methods (typically based on fluorescein dyes [487]). Highly selective and precise measurement techniques of liquid species include ion chromatography $\left(\mathrm{NO}_{2}^{-}, \mathrm{NO}_{3}^{-}\right)$[488], gas chromatography (gaseous $\mathrm{O}_{2}, \mathrm{H}_{2}$ stripped from the plasma treated liquid) [233], liquid chromatography (using chemical probes for detection of ${ }^{\bullet} \mathrm{OH}$, $\left.{ }^{\bullet} \mathrm{HO}_{2}\right)[489,490]$ or electron paramagnetic resonance spectroscopy (using spin trapping probes for detection of ${ }^{\bullet} \mathrm{OH}$ and superoxide radicals) [482].

Short-lived species require in situ and fast measurements, and performing these measurements presents challenges. However, even chemical analysis of long-lived chemical species in the liquid faces difficulties and requires special attention while working in complex liquid mixtures treated by plasma. Chemical composition of the surrounding environment (of both gas and liquid phases) strongly influences the physicalchemical properties of the plasma-treated liquid. Many of the chemical products in the liquid, especially those produced by air plasmas, are not stable. After the liquid has been treated by the plasma, reactions can continue to occur. These processes include catalytic reactions (e.g. $\mathrm{H}_{2} \mathrm{O}_{2}$ reacting with metal impurities sputtered from electrodes, such as $\mathrm{Fe}, \mathrm{Cu}, \mathrm{W}$,

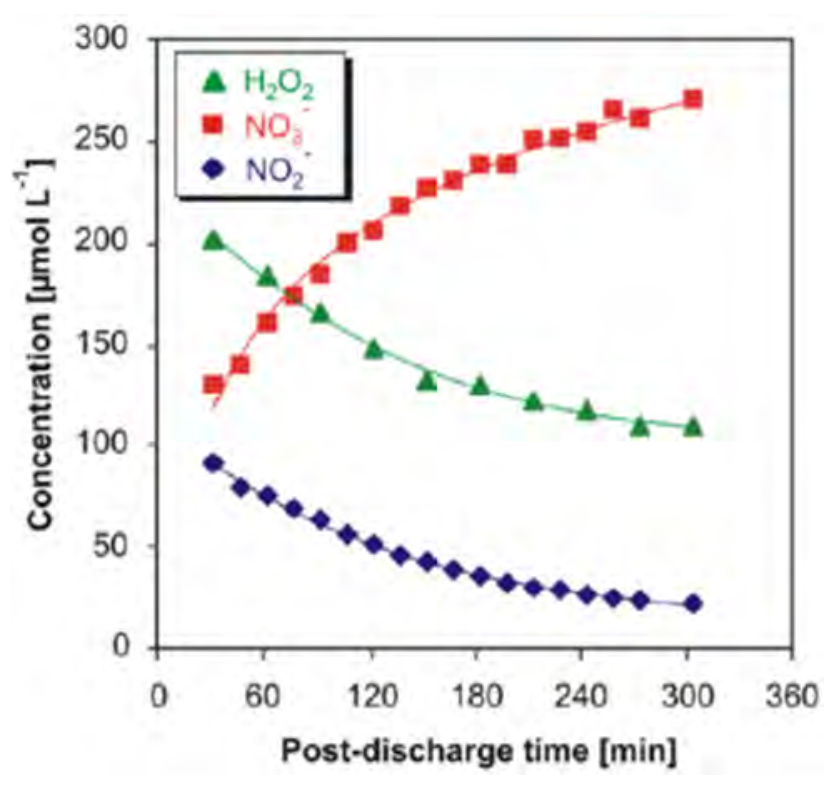

Figure 21. Post-discharge evolution of hydrogen peroxide, nitrite and nitrate in aqueous solution $(\mathrm{pH} 3.3)$ after treatment by an air plasma. Reproduced with permission from [488], copyright 2014 IOP Publishing.

Pt (equations (23) and (24))) [491-494], $\mathrm{pH}$ dependent processes (e.g. reaction between $\mathrm{H}_{2} \mathrm{O}_{2}$ and nitrites [495]), and decomposition of ozone under alkaline conditions (equation (26) and (27)) [496, 497]). Some of these reactions are:

$$
\begin{gathered}
\mathrm{Fe}^{2+} / \mathrm{Cu}^{+}+\mathrm{H}_{2} \mathrm{O}_{2} \rightarrow \mathrm{Fe}^{3+} / \mathrm{Cu}^{2+}+\mathrm{OH}^{\bullet}+\mathrm{OH}^{-} \\
\mathrm{WO}_{4}^{2-}+\mathrm{H}_{2} \mathrm{O}_{2} \rightarrow \mathrm{WO}_{5}^{2-}+\mathrm{H}_{2} \mathrm{O} \\
\mathrm{NO}_{2}^{-}+\mathrm{H}_{2} \mathrm{O}_{2}+\mathrm{H}^{+} \rightarrow \mathrm{NO}_{3}^{-}+\mathrm{H}_{2} \mathrm{O}+\mathrm{H}^{+} \\
\mathrm{O}_{3}+\mathrm{OH}^{-} \rightarrow \cdot \mathrm{OH}+\mathrm{HO}_{2}^{\cdot}+\mathrm{O}_{2}^{\cdot-} \\
\mathrm{O}_{3}+\mathrm{H}_{2} \mathrm{O}_{2} \rightarrow{ }^{\cdot} \mathrm{OH}+\mathrm{HO}_{2}^{\cdot}+\mathrm{O}_{2} .
\end{gathered}
$$

Therefore, care should be taken to properly measure chemical species produced in plasma treated liquid. Fixation of liquid samples removed from the continually reacting plasma treated liquid is an important step in an analytical procedure. This isolation can be accomplished by either radical quenchers or suitable buffers. For example, analytical methods to measure $\mathrm{H}_{2} \mathrm{O}_{2}$ in mixtures with nitrites are often performed under acidic conditions (e.g. iodometric, ammonium molybdate, metavanadate, titanium sulfate methods) [484, 498, 499]. These conditions and methods can lead to significantly lower values of $\mathrm{H}_{2} \mathrm{O}_{2}$ due to decomposition of $\mathrm{H}_{2} \mathrm{O}_{2}$ by nitrites upon acidification by a coloring reagent (equation (25)). This bias can be avoided by adding azide to the sample of $\mathrm{H}_{2} \mathrm{O}_{2}$ prior to mixing with the acidic analytic reagent [488].

$$
3 \mathrm{~N}_{3}^{-}+\mathrm{NO}_{2}^{-}+4 \mathrm{H}^{+} \rightarrow 5 \mathrm{~N}_{2}+2 \mathrm{H}_{2} \mathrm{O}
$$

Consequently, nitrites are not stable under acidic conditions and disproportionate to nitrates (equations (29) and (30)) [500] (see figure 21). This process can be eliminated 


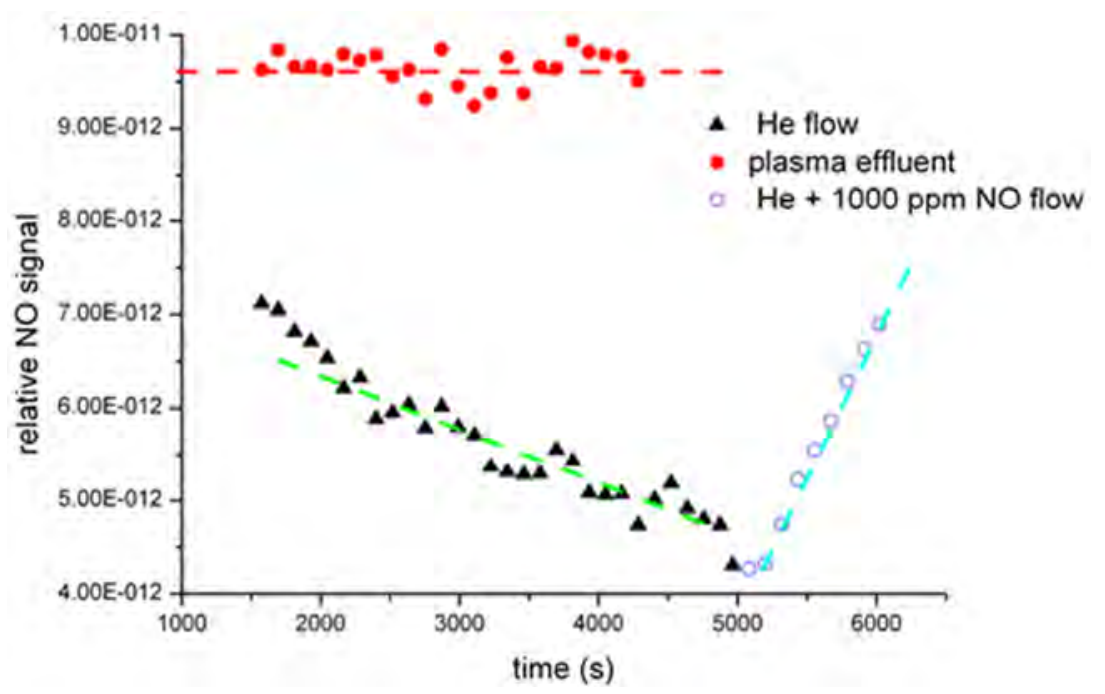

Figure 22. Mass spectrometry measurement of ${ }^{\circ} \mathrm{NO}$ build-up in the liquid phase for applied gas flow and plasma exposure. For He flow only we observe a decrease in time of the ${ }^{\circ} \mathrm{NO}$ signal due to the decrease of dissolved air components in the water. In the case of the plasma effluent this decrease is not observed and in the case of a flow containing $1000 \mathrm{ppm}{ }^{\bullet} \mathrm{NO}$ an increase in ${ }^{\bullet} \mathrm{NO}$ signal is observed with time. The example illustrates the potential of membrane mass spectrometry for liquid analyses $[529,530]$

by mixing the sample of plasma treated solution with basic buffer.

$$
\begin{gathered}
\mathrm{NO}_{2}^{-}+\mathrm{H}^{+} \leftrightarrow \mathrm{HNO}_{2} \\
2 \mathrm{HNO}_{2} \longrightarrow \cdot \mathrm{NO}+\mathrm{NO}_{2}^{\cdot}+\mathrm{H}_{2} \mathrm{O}
\end{gathered}
$$

Ozone dissolved in liquid is also not particularly stable and its stability is strongly $\mathrm{pH}$-dependent (equations (4) and (5)). The lifetime of peroxynitrite is typically less than $1 \mathrm{~s}$ in acidic solutions, which implies very low steady-state concentrations of peroxynitrite are likely present in plasma-treated water (nanomolar levels). It is therefore difficult to detect $\mathrm{ONOOH}$ in plasma treated water even if it is produced in large quantities during the plasma treatment. Attempts to identify peroxynitrite in plasma-treated water were made through absorption spectra [501, 502], using fluorogenic probes [503], and by analysis of the antibacterial activity of model solutions mimicking plasma-treated water [501, 502, 504]. Recently, the rate of formation of peroxynitrite in plasma-treated water was quantitatively determined through kinetic analysis of the post-discharge reaction between $\mathrm{H}_{2} \mathrm{O}_{2}$ and nitrite ions in air plasma-treated water at pH 3.3 (equations(31) and (32)) [505].

$$
\begin{gathered}
\mathrm{NO}_{2}^{-}+\mathrm{H}_{2} \mathrm{O}_{2}+\mathrm{H}^{+} \rightarrow \mathrm{O}=\mathrm{NOOH}+\mathrm{H}_{2} \mathrm{O} \\
\mathrm{O}=\mathrm{NOOH} \leftrightarrow{ }^{\bullet} \mathrm{OH}+{ }^{\cdot} \mathrm{NO}_{2}
\end{gathered}
$$

Since many of these processes are conducted in saline solutions, particularly for biological applications, the effect of the buffer needs to be accounted for. Recently the possible importance of chlorine chemistry has been suggested by Wende et al [77].

Radicals with their unpaired electron are paramagnetic. Electron paramagnetic resonance (EPR), also known as electron spin resonance (ESR) or electron magnetic resonance (EMR) spectroscopy is the most commonly used technique to determine radical densities. This method provides both qualitative and quantitative data. In the case of plasma-liquid-interactions, direct observation of many radicals is not possible because of their short lifetimes [506]. To overcome these limitations, EPR measurements can be enhanced using the spin trapping technique [507] in which the radicals are conjugated to specific spin trap compounds to prolong their lifetime by several orders of magnitude. The EPR spectra of these radical-spin-trap-adducts have characteristic finger print signatures which can be quantitatively analyzed $[508,509]$. One of the most frequently used spin traps for oxygen radicals is 5,5-dimethy-1- pyrroline-Noxide (DMPO) [510-519] which traps oxygen, nitrogen and carbon centered radicals.

The use of spin trapping assisted EPR measurements of plasma generated radicals in the liquid phase was first reported by Bullock et al who detected hydroxyl and hydrogen radicals in aqueous solution after glow-discharge electrolysis [520]. Hase and Harada also detected both radicals after aqueous solution treatment with a contact glow discharge at room temperature [521]. Both studies as well as recent reports have not indicated the absolute concentrations of the radicals [520-526].

Despite the potential advantages of using spin traps for monitoring short-lived species, care should also be used in the analysis. For example, reactions of spin traps with other species present in the media, spin trap and spin adduct stability, appropriate calibration, EPR instrumentation parameters, compound variations due to different manufacturers of the spin trap or the effect of the time delay between treatment and measurement [482] are all variables that need to be controlled. Different liquids can also change the results, not only due to different reaction pathways but also due to perhaps inherent scavenging capacity [483]. For example, ethanol is known to have a hydroxyl radical scavenging [527] capability while also yielding additional radical generation sources. Another drawback of EPR spectroscopy is that only the averaged concentration in the total volume can be determined, since time and spatially resolved measurements cannot be performed. 
Short-lived RNS formed by plasma activation, such as • $\mathrm{NO}$ and ${ }^{\bullet} \mathrm{NO}_{2}$, undergo chemical reactions in the presence of water and oxygen, producing nitrite and nitrate anions in the liquid phase. To the best of our knowledge, there are no direct measurements of nitric oxide and nitrogen dioxide in liquids exposed to plasma. Experimental detection of RNS in the liquid phase presents an important yet challenging task in order to fully understand the complex chemistry of low temperature plasma-treated liquids. A combination of synthetic organic chemistry methods with physical chemistry techniques could ultimately enable measurements of such species both in bulk liquid and in plasma/liquid interfacial layers. In this context, Bruggeman et al in collaboration with Hiden Analytical measured dissolved ${ }^{\bullet} \mathrm{NO}$ in the liquid by mass spectrometry through a porous membrane in liquid (see figure 22) [528].

One of the methods of choice to analyze elemental components in liquids is injection of aerosols into ICPs, vaporization of the aerosol and use of OES or mass spectroscopy to measure the dissolved components. This method is able to detect metallic and some non-metallic elements in concentrations as low as parts-per-trillion (ppt). Mass spectroscopy also enables isotopic speciation. However, this technique is not able to differentiate between the valence state of metals (e.g. $\mathrm{Cu}^{+}$or $\mathrm{Cu}^{2+}$ ), which might be necessary for accurately identifying reactions in the bulk of the treated liquid. A complementary method to provide information on valence states could be electrospray ionization with mass spectroscopy (ESI-MS) [9].

Many researchers are interested in the chemical action of reactive species on complex chemical components in the context of plasma initiated degradation of pollutants in water, usually by oxidation. Among the analytical techniques used to study and characterize the liquid phase in this area, high performance liquid chromatography (HPLC) coupled to mass spectrometry provides keen insights on the mechanism of degradation [531]. Identifying the reaction intermediates also gives indirect information on the species involved in the oxidation process. For example, the detection of nitrated intermediates in the phenol decomposition by air plasma gives an indication of the nitrating agents produced by the plasma [488]. However, this indirect way to detect reactive species must be used carefully. For example, cis, cis-muconic acid is sometimes cited as a specific product of $\mathrm{O}_{3}$ reactions with phenol. However, muconic acid can also be produced by breaking the aromatic ring of cathecol, which is formed by the reaction of phenol with ${ }^{\bullet} \mathrm{OH}[532,533]$. Thus, the correlation of $\mathrm{O}_{3}$ initiated oxidation with the presence of cis, cis-muconic acid should be made with care.

HPLC enables separation of each component of the solution and mass spectrometry provides the molecular mass and, most importantly, the structure of each component detected through tandem MS/MS experiments. So by mapping the various reaction intermediates it is possible to infer the pathway of the degradation of pollutants. Often ions with higher $m / z$ ratio than the molecular ion of the parent compounds are revealed. It is not always straightforward to identify these structures. In fact, heavy ions can be produced by the addition of $\mathrm{O}$ atoms to the molecule [534] or by oxidation and rearrangement of the molecule [535]. The formation of coupling products is also possible but these are thought to only be products of the Fenton process [536]. Nuclear magnetic resonance (NMR) spectroscopy can be used to identify many stable chemical species formed from complex mixtures of organic compounds from plasma-liquid systems [537].

In conclusion, there is great need for specific analytical techniques for detection of chemical species produced by plasmas in liquid environments. However, analytics of plasma induced chemistry in aqueous liquids are sensitive to the complexity of the resulting solution containing organics, impurities, or species of high ionic strength. These conditions significantly influence selectivity, sensitivity and precision of the analytical methods. Diagnostics of long-lived products can be performed by selecting the most suitable analytical procedure and eliminating possible interferences by fixation of liquid samples by buffers, radical quenchers or other modifiers. Nevertheless investigators should always keep in mind that adding chemicals to detect species might significantly alter the chemical balance between species, particularly when the probe reacts with the molecule it detects. In many cases the measurements interferes with the solution under study.

On the other hand, in situ diagnostics of short-lived species in plasma treated liquids constitute a great challenge. In addition to the direct quantitative measurements such diagnostics provide, indirect analytical methods which are based on chemical probes of radicals (e.g. spin traps using EPR, fluorescence dyes for ROS, detection of specific products by chemical probes) show great potential for the analysis of decay kinetics and reaction pathways of long-lived products in plasma treated liquid.

While the selectivity of a probe against long-lived reactive plasma species, such as ozone or hydrogen peroxide, can be studied, selectivity experiments against short lived reactive species are more complicated because of the lack of a readily available quantitative source of these species. These species are traditionally created chemically and the conditions required to synthesize these species may also affect the probe itself. It may also be problematic to detect small amounts of a reactive species in the presence of large quantities of other reactive species because the technique does not have infinite selectivity. It is often the minority species that has a major effect on, for example, plasma induced biological processes.

While probe characterization is often conducted under well-defined and controlled conditions, the calibration may not apply to plasma induced processes. For example $\mathrm{pH}$ and temperature are likely to change during the experiment and may have significant spatial variation [62]. There are no guidelines on how to account for these variations when making in situ measurements. Some probes, however, have broad pH stability [487] and, therefore, may be more suited for scenarios in which $\mathrm{pH}$ changes are expected to occur. Attention should also be given to the reactivity and stability of the reaction products of the probe molecules. For example, chemical probes designed for ozone detection may yield fluorescent products that could undergo further reactions with ozone (or other reactive plasma species) thereby challenging quantitative analysis [538]. 


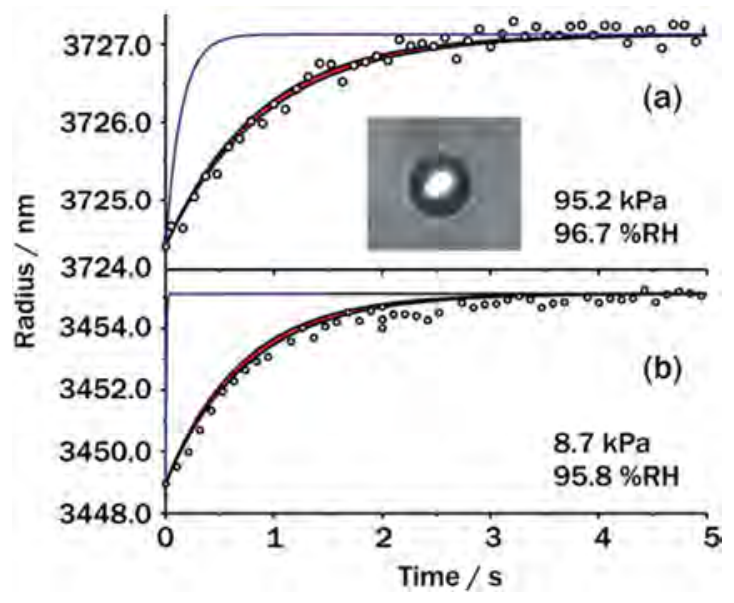

Figure 23. Examples of the measurement of condensation kinetics of water on an optically trapped aqueous droplet (inset) at two different gas pressures and relative humidity. The droplet size is measured (points) with high accuracy from the cavity enhanced Raman spectrum and the condensation process initiated by the removal of a laser beam driving optical heating. Simulations of the kinetics must include the influence of slow heat transfer away from the droplet during condensation (red line), not just the fast diffusional mass transport (blue line). Adapted from [545].

A set of reference analytical methods for commonly produced reactive species in liquids would help in the evaluation and comparison of different types of plasma systems, and lead to an improvement in our understanding of the general mechanism of chemical processes induced by plasmas in liquids. A preliminary list of such discussed at the Lorentz center workshop is in table A2 in the appendix. A source of analytical techniques can be found in biochemistry and physiology as there are analogous processes to those occurring in plasmaliquid interaction [539, 540].

\section{Diagnostics of gas-liquid interfaces}

A wide range of analytical tools have been developed over many years to probe the structure of solid surfaces and the dynamics of chemical transformations on solid substrates. Characterizing chemical transformations and dynamics at liquid surfaces is considerably more challenging. The evaporation of liquid into the gas phase precludes measurements under the ultrahigh vacuum conditions typically used in surface science and the continual turnover of material at the surface on nanosecond timescales leads to dynamic surfaces that evolve with time. Chemical reactions can occur over a finite depth, often characterized by the reaction-diffusive length, which depends on the rate of chemical reactions beneath the surface. There are few analogues to these conditions in traditional surface science of solids where most reactions occur directly at the surface. Despite these challenges, diagnostic tools have been developed for studying liquid surfaces which rely predominantly on either the scattering of light or molecules from the liquid surface $[156,541,542]$. In this section we will not discuss conventional techniques that allow measurements of the macroscopic quasi-steady/equilibrium properties of liquid surfaces (for example, surface tension), but will instead focus on techniques that probe their microscopic structure and dynamics.

Optical spectroscopy can be used to probe the gas-liquid interface directly through a range of surface sensitive techniques. Alternatively, the kinetics of processes occurring at the interface can be inferred from measuring changes in gas or liquid phase composition. One widely used technique to probe gas-liquid exchange of reactive and non-reactive species is the droplettrain approach [156, 541]. Droplets of $\approx 100 \mu \mathrm{m}$ diameter are generated in a train by a vibrating orifice aerosol generator. The depletion in the gas phase concentration of trace species is probed by tunable diode laser infrared spectroscopy over a time extending up to $20 \mathrm{~ms}$. By varying either the interaction time or the size of the monodisperse droplets, the gas-phase depletion and the uptake coefficient characterizing the kinetics of gas-particle exchange across the interface can be inferred. The kinetics of interfacial exchange have been studied for a wide range of soluble species on aqueous, organic and sulphuric acid droplets [156]. In a variation of this technique, the depletion of a gas phase species can be measured by mass spectrometry or optical spectroscopy to study the uptake of non-reactive and reactive species by aqueous, organic and inorganic crystalline monodisperse or polydisperse aerosols over seconds or minutes [541]. When coupled with end-of-the-line sample collection and analysis by techniques such as soft vacuum ultraviolet photons, the chemical processing that occurs through gas-liquid exchange of radicals including ${ }^{\circ} \mathrm{OH}$ can be characterized [543].

Studies of reactive chemistry have also been performed on single particles held in optical or electrodynamic traps with measurements of uptake coefficients, product branching and the physicochemical properties of the processed particle. Often these measurements have concentrated on ozonolysis chemistry of droplets containing olefinic bonds [544]. With the high precision in size and refractive index enabled by cavity enhanced Raman scattering from trapped droplets or from angular light scattering profiles, the kinetics of water transport can be probed. These techniques extend to measuring the kinetics of the condensation or evaporation of sub-nanometer layers of water at the surface of an aqueous droplet (see figure 23) [545]. The influence of surfactant monolayers on the kinetics of transport across the liquid-gas surface has been studied on single particles, demonstrating that monolayer coverages of a surface can significantly impair transport through the interfacial region [546]. Evaporation kinetics from a liquid surface have also been measured using liquid microjets. For example, Saykally et al have inferred the evaporation coefficient of water from an aqueous surface by measuring the time-dependence of the temperature of the jet during evaporative cooling using Raman thermometry [547]. By coupling a liquid microjet into the spraying chamber of an electrospray ionization mass spectrometer, the mechanisms and product branching of interfacial reactions can be studied [548].

Although studies on droplets or jets enable surfaces to be readily renewed, measurements of uptake coefficients have been made on flat surfaces, again typically by measuring depletion of gas phase reactant concentrations [156]. These techniques have been used to probe reactive uptake coefficients of radical species such as ${ }^{\bullet} \mathrm{OH}, \mathrm{HO}_{2}{ }^{\bullet}$ and ${ }^{\bullet} \mathrm{NO}_{3}$. 


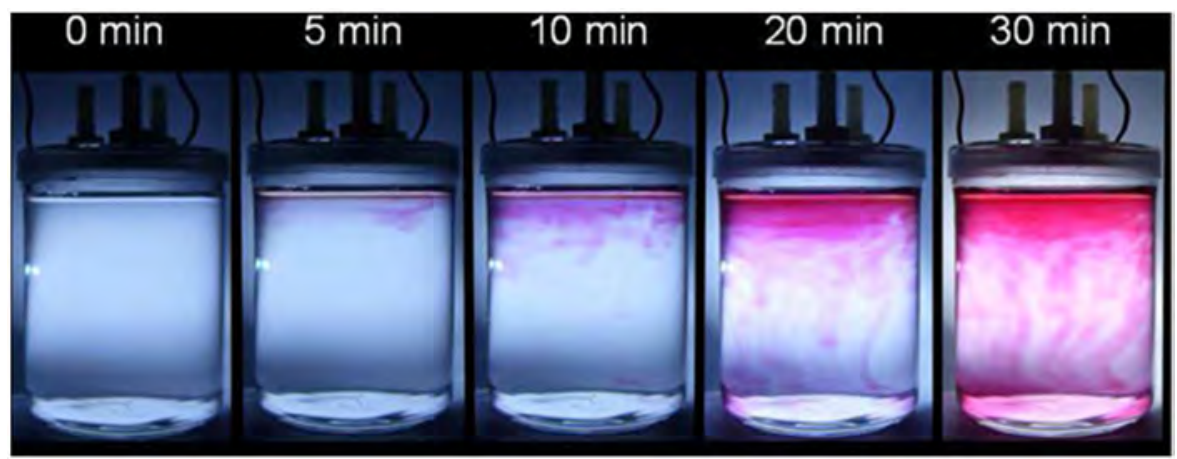

Figure 24. Liquid volume treatment by a surface $\mathrm{DBD}$ leading to the formation of $\mathrm{NO}_{2}^{-}$in the liquid as shown by the formation a magenta colored azo dye. No flow is present in this case so the redistribution of the dye is due to diffusion of $\mathrm{NO}_{2}^{-}$and/or the dye in the liquid volume. Reproduced with permission from [293], copyright 2011 IEEE.

Donaldson et al used LIF coupled with surface active chromophoric molecules such as polyaromatic hydrocarbons (PAHs) to investigate chemical processes at flat liquid surfaces. By exciting PAHs in the UV with a glancing angle laser probe beam, the fluorescence spectrum provides a direct signature of the local polarity of the probe chromophore at the interface [549]. By measuring the time-dependence of fluorescence intensity, the kinetics of reactant adsorption (e.g. ozone) at the liquid-gas surface can be inferred, with the kinetics often consistent with a Langmuir-Hinshelwood mechanism.

These studies are typically performed under well controlled conditions. Plasmas deliver a variety of reactive neutral species to surfaces and in addition to depositing charge. In most cases a large flux of (V)UV photons is present, particularly for plasmas in contact with the interface. This complex mix of species not only reacts in the gas phase but is unlikely to selectively react on assistant-agents such as dyes or chemicals used to track chemistry in the liquid phase. Studies of well-defined transfer from gas-to-liquid phase by examining, for example, droplets of well-defined sizes in a homogeneous gas composition could be a point of departure for the plasma community. Valuable work has also performed in the area of electrospray mass spectrometry.

To characterize the equilibrium composition of the liquidgas surface, a number of advanced optical tools are available, including photoelectron spectroscopy, non-linear second harmonic generation (SHG) and sum-frequency generation (SFG) spectroscopy. These methods rely on the fact that for centro-symmetric materials such as water or air the second order susceptibility, $\chi^{(2)}=0$. At the interface between the two materials, the symmetry is broken yielding $\chi^{(2)} \neq 0$. SHG or SFG signals can then be produced. Their surface selectivity can be sub-monolayer. However, with a large number of species and with high electric fields present at the interface, gaining quantitative information using these techniques may be difficult. On the other hand, electric field-induced SHG has been widely used to probe hyper-polarizabilities of molecular species and may be a useful tool to probe local effects induced by the electric field at the plasma-water interface [550]. SFG experiments could be used to probe the hydrogen bonding structure of water at the interface. Individual species may potentially be probed by SHG or SFG tuned to specific electronic or vibrational transitions to gain some species selectivity through resonance-enhancement. A wellstudied example also of interest to the plasma community is solvated electrons [224]. Recently, total reflectance absorption spectroscopy has been used to measure solvated electrons at a plasma-liquid interface [199]. Using SFG, Kondo and Ito recently investigated the effect of an externally applied electric field on the orientation and polarization of water molecules at an insulator-water interface [551].

X-ray photoelectron spectroscopy (XPS) can be used to probe, as a function of depth, the near-surface composition of liquid microjets or a static sample. These data can yield insights on the partitioning of anions and cations within the first few nm of the surface $[552,553]$. Ammann and coworkers extended this approach to measure composition profiles over depths of a few nanometers for ice surfaces using ambient pressure XPS and more recently for the liquidvapor surface of aqueous salt solutions of formic acid [554]. When measurements over a few nanometers are compared with measurements of the top surface layer by non-linear spectroscopy, a comprehensive picture of the liquid-gas surface emerges. Indeed, it is possible to directly probe the evolving composition of the surface, demonstrated by a monolayer of oleic acid reacting with ozone adsorbing from the gas phase [555].

To complement these spectroscopic tools, it is now possible to directly measure the interaction of molecules colliding with a liquid surface using molecular beam techniques. Nathanson and coworkers directed a beam of molecules (e.g. $\mathrm{HCl}, \mathrm{DCl}$ ) onto a constantly renewed liquid surface on a rotating disk [556]. They measured the branching of inelastic scattering, trapping and desorption of molecules. In such measurements, the incident molecules have a kinetic energy considerably higher than the surface temperature. Measurements must be made on liquids of low volatility such as concentrated sulphuric acid or liquid glycerol. Minton [557] and McKendrick [558, 559] examined the collisions of superthermal oxygen atoms and hydroxyl radicals with a squalane surface. They reported quantum state resolved product distributions, a technique that can also be used to study reactions on self-assembled monolayers. 
Electro-chemical techniques capable of probing interfacial processes are also possible diagnostics [560]. Electrochemical techniques consist of measuring potentials, current and charge based on inserting electrodes in the system of study, techniques common in the field of plasmas. However the cathode or anode voltage drops when a plasma is in contact with the liquid are of the order of $200-600 \mathrm{~V}$ and might dominate the typical potentials of $1 \mathrm{~V}$ for electrochemical processes.

In conclusion, while a large set of diagnostics is available to measure individual processes that are important to plasma-liquid interactions, the inherent complex conditions complicate their implementation. Nonetheless, wellcontrolled techniques assessing fundamental properties such as accommodation coefficients and chemical reactions at liquid gas interfaces will be valuable in providing the fundamental data required for models of plasma-liquid interactions.

\subsection{Diagnostics for macroscopic gas-liquid transport and interfacial dynamics}

Measuring flow and transport of species at the gas liquid interface is extremely challenging. Flow patterns are often measured by laser scattering or fluorescent probe techniques. The change in refractive index between the liquid and the water phase leads to scatter and diffraction which may complicate accurate interfacial measurements. The highly dynamic plasma-liquid interface causes exceptional challenges for high resolution gas and liquid phase diagnostics. Deformation of plasma-liquid interfaces has been studied using fast imaging, including Taylor cone formation [561, 562], wave phenomena at the gas-liquid interface, and boiling phenomena induced by plasma [295, 563].

Gas and liquid phase transport have been measured using different approaches. In both the gas and liquid phase, Schlieren [467], shadowgraphy [564], particle imaging velocimetry (PIV) [311] and fluorescence measurements of a molecular probe added to the flow [302] have been used. The last two techniques enable spatially resolved velocity flow field measurements while the first two techniques are based on optical differences and provide information on the flow structure. In the liquid phase, scattering from particles [311, 565] and on microbubbles [301] can be used to measure flow fields. Note that fluorescent probe molecules might not be compatible with plasma treatment as they could be oxidized and lose their specific fluorescent properties.

Several studies have been performed by observing discoloration of dyes or adding a non-colored chemical which reacts with plasma produced species to create a colored reaction product. An example is shown in figure 24. Similar techniques have also been applied in sonochemiluminescence [566]. $\mathrm{pH}$ sensitive dyes have also been used [293, 567]. It should be stressed, as indicated above, that selectivity of the chemical process should be carefully checked.

Only a limited number of studies on gas and liquid phase transport for plasma-liquid systems have been reported in the literature. This is an important area for understanding plasma-liquid interactions, particularly for understanding

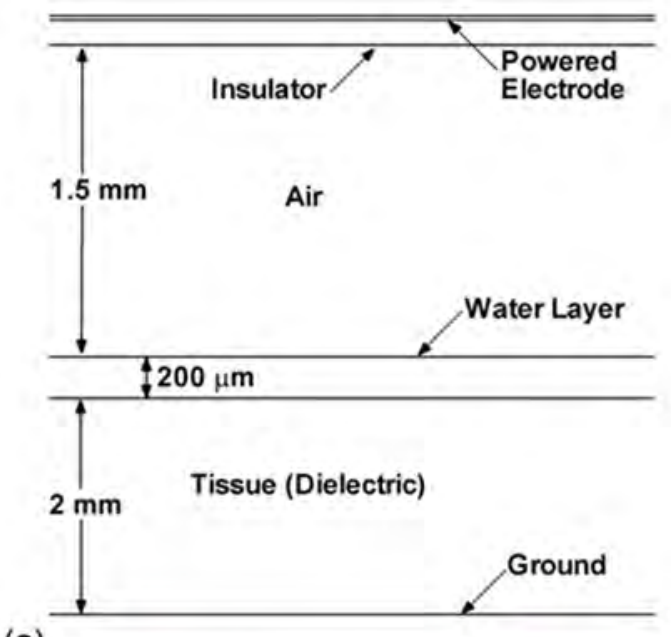

(a)

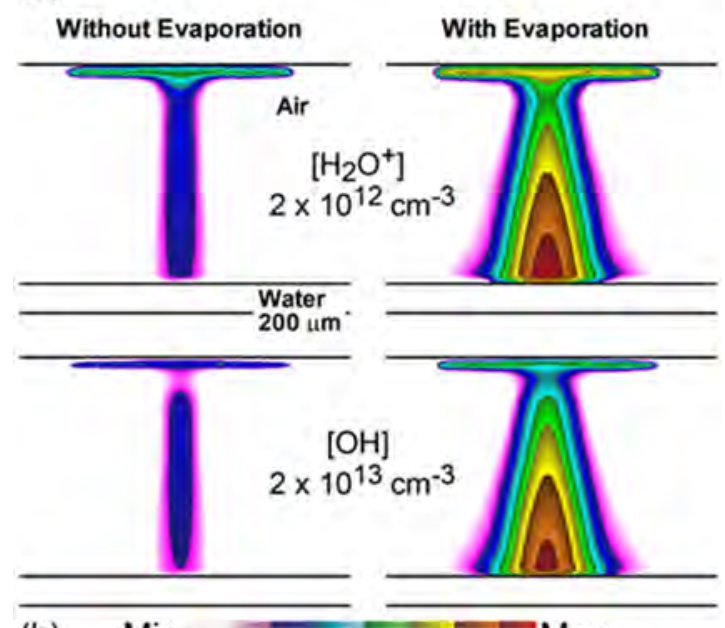

(b)

Figure 25. Dielectric barrier discharge filaments interacting with thin water layers over tissue (a) geometry, showing $1.5 \mathrm{~mm}$ gas gap over a $200 \mu \mathrm{m}$ water layer. The water evaporates into the gap. (b) Densities of $\mathrm{H}_{2} \mathrm{O}^{+}$and ${ }^{\bullet} \mathrm{OH}$ in the discharge with and without evaporation of water vapor. The initial gas mixture is $1 \mathrm{~atm}, \mathrm{~N}_{2} / \mathrm{O}_{2} /$ $\mathrm{H}_{2} \mathrm{O}=79.9 / 20 / 0.1$. Contours for $\mathrm{H}_{2} \mathrm{O}^{+}$and ${ }^{\bullet} \mathrm{OH}$ are plotted on a log scale over three decades with the maximum density shown in each frame. Reproduced with permission from [62], copyright 2014 IOP Publishing.

the transfer of reactivity originating in the plasma to the liquid phase in small volumes of liquids or thin liquid layers.

\section{Modeling}

\subsection{Introduction}

The modeling and simulation of plasma-liquid interactions is challenging from a number of perspectives. The first is the large dynamic range in collisionality between the gas phase plasma and the liquid phase plasma which challenges the numerical integration of gas and liquid phase processes. The second is the uncertainty in reaction mechanisms, less so in the gas phase and more so in the liquid. The third is the manner of transport of gas phase species into the liquid and from the liquid into the gas phase. In 


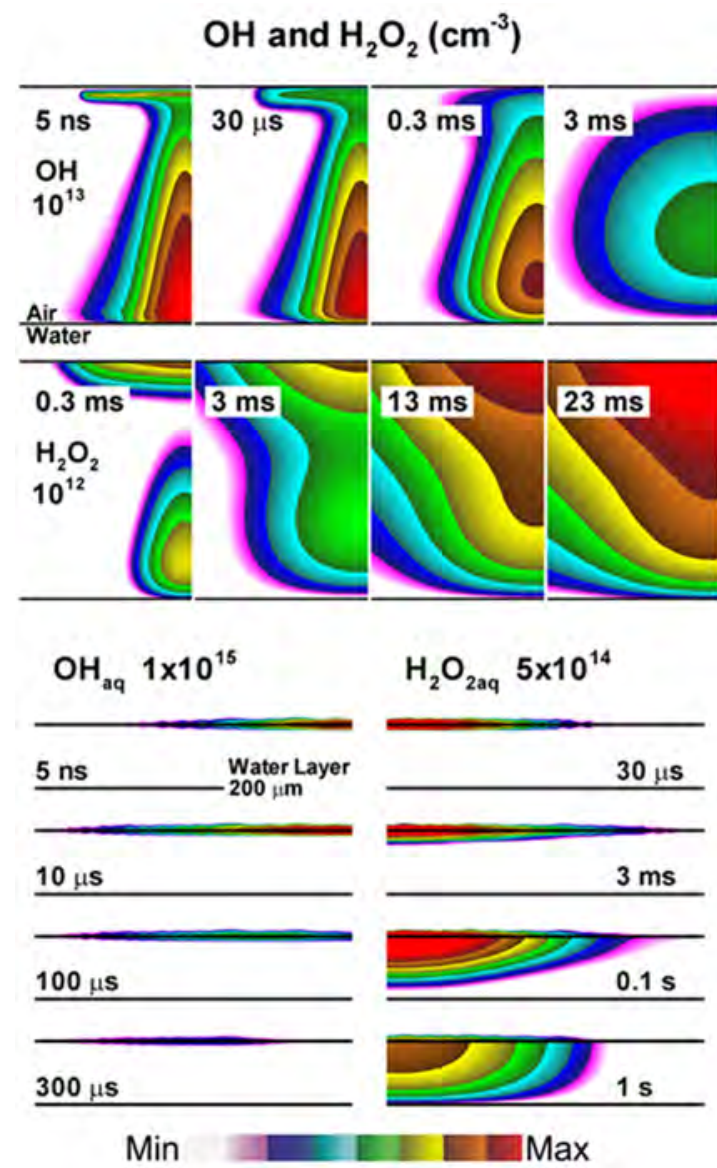

Figure 26. The time evolution of $\mathrm{OH}$ and $\mathrm{H}_{2} \mathrm{O}_{2}$ densities in the (top) gas gap (initial gas mixture is $1 \mathrm{~atm}, \mathrm{~N}_{2} / \mathrm{O}_{2} / \mathrm{H}_{2} \mathrm{O}=79.9 / 20 / 0.1$ with water evaporation) and (bottom) $200 \mu \mathrm{m}$ water layer. Densities of ${ }^{\bullet} \mathrm{OH}$ and ${ }^{\bullet} \mathrm{OH}_{(\mathrm{aq})}$ are shown during the first pulse of $-18 \mathrm{kV}$ and interpulse afterglow. $\mathrm{H}_{2} \mathrm{O}_{2}$ and $\mathrm{H}_{2} \mathrm{O}_{2(\mathrm{aq})}$ densities are shown accumulating after 3 successive pulses at $100 \mathrm{~Hz}(0,100 \mathrm{~ms}, 200 \mathrm{~ms})$ and during the terminal afterglow. The contours are plotted on a 3-decade log-scale with the maximum values noted in each frame. Reproduced with permission from [62], copyright 2014 IOP Publishing.

the discussion that follows, we will discuss two classes of models-those addressing gas phase plasmas-interacting with liquids and those addressing plasmas-in-liquids. In spite of the challenges for modeling plasma-liquid interactions, significant progress has been made addressing the coupled systems of atmospheric pressure plasmas and liquid phase reactivity [61, 62, 188, 323, 568].

\subsection{Gas phase plasmas interacting with liquid}

In this section we discuss the case when plasma is actively generated in the gas phase-the voltages and electric fields being applied are too small to sustain or to independently generate plasma in the liquid. The reactive species are first produced in an atmospheric pressure plasma in the gas phase, these species transport to the plasma-liquid interface, penetrate through it and subsequently react in the liquid.

Transport through the plasma-liquid interface in principle occurs both from the plasma into the liquid, and from the liquid into the plasma. It is common to limit the transport of neutral species into (and out of) the liquid by some variation of a Henry's law equilibrium. In a spatially dependent model, Henry's law can be used to limit the rate of diffusion from the gas phase into the top layer of the liquid (or out of the liquid if the neutral species is over dense in the liquid) based on the densities of the neutral species directly above-and-below the plasma-liquid interface. This technique seems reasonable for quasi-steady state modeling since the asymptotic value at long times provides the required equilibrium densities. It is not clear if this approach is accurate during transients and on short time scales. Kinetic or molecular dynamics modeling would provide insights into the applicability of the Henry's law approach [569].

These techniques best apply to a species that has a small density in the liquid and a large density in the gas phase, such as dissolved $\mathrm{O}_{2}$ or $\mathrm{CO}_{2}$. In this case, the net flux is usually from the gas phase through the plasma-liquid interface into the liquid. This is also the case for minor species that are produced on a transient basis in the gas phase, such as ${ }^{\bullet} \mathrm{OH}, \mathrm{H}_{2} \mathrm{O}_{2}$ or $\mathrm{HNO}_{x}$. Since the densities in the gas phase are much larger than their equilibrium values in the liquid, the net transport is from the gas phase into the liquid. Once in the liquid, many of these species will react and be converted into other species, and so their effective densities are small. So the net flux continues to be from the gas into the liquid.

The transport of major species from the liquid into the gas phase, such as the water itself, is typically addressed from the thermodynamic perspective of evaporation - the liquid has a vapor pressure appearing as a diffusion source of the molecules composing the liquid into the gas phase. This results in there being a layer of gas adjacent to the liquid that is saturated with the vapor of the liquid. This saturated layer is very important in gas phase plasmas interacting with a liquid as plasma generated radicals and ions react with the saturated water vapor on top of the liquid prior to reaching the surface of the liquid. The reactions include electron impact dissociation and ionization that can produce, for example, ${ }^{\bullet} \mathrm{OH}$ in close proximity to the water, as well as charge exchange reactions that either hydrate the gas phase ions or convert most gas phase ions into water ions [445]. An example of the calculation of the gas phase ${ }^{\bullet} \mathrm{OH}$ and $\mathrm{H}_{2} \mathrm{O}^{+}$density produced in a DBD interacting with a liquid layer with and without considering evaporation from the liquid layer is shown in figure 25. This figure clearly illustrates the large impact of evaporation on the plasma kinetics.

The just described method of addressing the plasma liquid interface does not necessarily acknowledge physical or chemical barriers or enhancements to transport on either side of the gas-liquid interface. There certainly are classes of hydrophobic molecules that resist penetration through the plasma liquid interface or surfactants that will be attracted to the interface. Charged species and photons may require a specific treatment (see sections 3 and 4). An alternative method of modeling the plasma-liquid interface, and perhaps better prepared to address these physio-chemical effects, is the use of an adsorbed layer. That is, instead of species directly transporting from the gas into the liquid, these species first adsorb onto the liquid surface if coming from the gas phase or are attracted to the interface if coming from the liquid phase. Adsorbed 
species then transport into or out of the liquid based on their own interfacial transport properties. Reactions can also occur between adsorbed species and between gas phase and adsorbed species. This technique is analogous to the method used to model plasmas in contact with solids where gas phase species can chemisorb or physisorb, and can be mobile on the surface. This technique has been fully developed in the modeling of the uptake of gases into aerosols for the investigation of atmospheric chemistry [159].

The interaction of a gas molecules or ions with surfaces of aerosol particles or bulk liquid involves multiple transport and kinetic processes [156]. An explicit approach describing such processes $[157,159]$ is shown in figure 9, which is a schematic of the key processes and flux terms used to describe gas uptake by atmospheric particles. Heat flux can also be included in this framework. Note that in most cases in aerosol chemistry except water condensation, a temperature change in the aerosol particles is negligible, as aerosol particles are well buffered by surrounding gases [140, 159].

Two examples of modeling the transfer of ${ }^{\bullet} \mathrm{OH}$ and $\mathrm{H}_{2} \mathrm{O}_{2}$ in a DBD plasma and of ROS in an $\mathrm{He}-\mathrm{O}_{2}$ plasma from the gaseous plasma phase to the liquid interface are shown in figures 26 and 27 respectively. These results clearly illustrate the limited penetration of short lived species such as ${ }^{\bullet} \mathrm{OH}, \mathrm{O}_{2}^{\bullet-}$ and $\mathrm{HO}_{2}^{\circ}$ and their conversion into $\mathrm{H}_{2} \mathrm{O}_{2}$. Note that in the presence of air, the kinetics becomes considerably more complex than shown in figure 27.

In principle, the same modeling techniques can be used to address plasmas in intentionally produced macroscopic bubbles that have been injected into the liquid as used to model gas phase plasmas $[114,570]$. In both cases, there is distinct gas phase plasma in contact with a liquid, albeit with a different, more tightly coupled geometry. The deformation of the bubble by plasma forces (which could also occur for a noncurved gas-liquid interface) is an important consideration in these systems and so should be addressed. Plasmas can also be sustained in bubbles that are thermodynamically produced in the liquid through heating the liquid. Here, the phase change between the liquid and the gas must be included in the thermodynamics and the model becomes a truly multiphase model. However, once the bubble is formed, the plasma is sustained in the gas phase of the bubble. From a modeling perspective, this is very similar to the injected bubble case.

\subsection{Plasmas in liquids}

Plasmas in liquids refer to those systems where the voltage, electric fields or other conditions enable the formation of plasma directly in the liquid phase. Although technologically very important from many perspectives, the fundamental processes of initiating and sustaining plasma in liquids are poorly understood.

Plasmas in bubbles in liquids are an important subset of plasma in liquids. Again, there are two approaches that require different modeling techniques. The first technique for selfsustained plasmas in liquids relies on the existence or formation of bubbles to provide a low density in the liquid phase which, for a given electric field, amplifies the local $E / N$ to the degree that a plasma can be sustained. The initiating bubbles are believed to be only a few microns in size, and are either pre-existing or are produced by the electrostriction effect due to large electric fields $[30,571,572]$. It is still unclear how these forces can grow a large enough bubble to provide the critical $p d$ (pressure $\times$ diameter) that will enable avalanching of the gas inside the bubble. Modeling the formation of the micro-bubbles requires advances in our understanding of this process. There may be techniques that can be adapted from the modeling of cavitation in CFD. The second class of plasma in liquids is the self-sustained discharge in liquid in the absence of bubbles. This discharge is produced by applying a large enough electric field that even with the liquid density, the $E / N$ is large enough to sustain the plasma [573]. Although the electron and ion transport coefficients of the in-liquid selfsustained discharge are poorly known, the mechanics of the modeling of this process should not be significantly different from modeling of ionization waves in the gas phase. The time and spatial scales are, however, quite different and may require different mathematical techniques. The groups of Joshi and Schoenbach performed pioneering work in this area [34, 574], as have Locke and Thagard [38].

\subsection{Reaction mechanisms}

The modeling of plasmas interacting with liquids require two somewhat independent sets of reaction mechanisms-for gas phase and for liquid phase properties. The reaction mechanisms are in principle independent but are interdependent with respect to transport through the plasma-liquid interface. If the plasma-liquid interface is addressed as an adsorbed layer, then a third reaction mechanism is required to described the reaction kinetics (and transport) of species resident into, out of and in the adsorbed layer. Many of the relevant mechanisms and data sets are discussed in sections 3 and 6 .

In modeling the reaction mechanism in the liquids, it is important to include only the elementary equations associated with the chemical species of interest [575]. In the modeling of gas phase plasmas, there is a generally accepted format for rate coefficients based on fundamental cross sections and distribution functions. The situation is less well defined in liquids due in large part to the difficulty of making quantitative measurements in real time, a capability that is available for gas phase measurements. Due to the hugely different densities of the liquid, for example water, compared to the reactive species, it is often assumed that water molecules are an infinite reservoir whose density is not affected by the chemical reactions. In these cases, reactions quoted in the literature often do not have a mass balance between reactants and products because of the assumed availability of water molecules from the infinite bath.

When one refers to several different sources and data bases to develop reaction mechanism, care must be taken to make sure that the quoted reactions are not redundant or do not include combined reaction pathways of other elementary reaction pathways. The status of the reaction mechanisms 
for the liquid is discussed in section 6 . To build a computational model based on such macroscopic equations for reactions and diffusion, using correct reaction rates and diffusion coefficients [576] (as well mobility coefficients) is essential to obtain reliable computational results. For some reactions (especially those involving organic molecules), reaction rates may not be available. One way to estimate such reaction rates may be molecular based simulation such as quantum mechanical calculations for multiple molecules.

Very often rate coefficients are quoted for buffered solutions in which, for example, the $\mathrm{pH}$ is constant. In these situations, it is usually assumed that there is an infinite reservoir of, for example, $\mathrm{H}_{3} \mathrm{O}^{+}$and $\mathrm{NO}_{3}^{-}$in the case of a nitric acid buffered solution. (Note that in much of the electrochemistry literature $\mathrm{H}_{3} \mathrm{O}^{+}$is referred to as $\mathrm{H}^{+}$with the implicit assumption that the $\mathrm{H}^{+}$is actually hydrated. In this review, we use the $\mathrm{H}_{3} \mathrm{O}^{+}$notation.) Recall that the $\mathrm{pH}$ of a solution is typically given by $\mathrm{pH}=-\log _{10}\left[\mathrm{H}_{3} \mathrm{O}^{+}\right]$where $\left[\mathrm{H}_{3} \mathrm{O}^{+}\right]$is the molar concentration $\left(\mathrm{mol} \mathrm{l}^{-1}\right)$. So a $\mathrm{pH}$ dependent rate coefficient is actually a multi-body rate coefficient where the constant density of $\mathrm{H}_{3} \mathrm{O}^{+}$is suppressed. Since plasma treatment of liquids can significantly change the $\mathrm{pH}$ of the water through formation of $\mathrm{H}_{3} \mathrm{O}^{+}$, reaction rate coefficients that depend on $\mathrm{pH}$ should be expressed as a function of hydronium $\left[\mathrm{H}_{3} \mathrm{O}^{+}\right]$ to make those rate coefficients implicitly dependent on the current conditions. Many reaction rates are only provided for specific $\mathrm{pH}$ values.

For the majority of models of plasma liquid interactions, non-equilibrium continuum techniques are likely adequate. That is, kinetic algorithms are not necessarily required. This is certainly the case in the liquid. The exception is in the sheath at the surface of the liquid where the plasma produced ionization wave strikes the surface. The $E / N$ produced in the sheath at the surface of the liquid may exceed thousands of $\mathrm{Td}\left(1 \mathrm{Td}=10^{-17} \mathrm{~V} \mathrm{~cm}^{2}\right)$-electric fields of hundreds of $\mathrm{kV} \mathrm{cm}^{-1}$. For positive discharges, this large electric field at the liquid phase boundary may produce moderately energy ions (up to $10 \mathrm{~s}$ of eV) onto the liquid surface in spite of the short mean-free-path at atmospheric pressure. The availability of reaction rate coefficients for ions of these energies onto water is very limited. In view of uncertainties in many of these rate coefficients it is important to perform uncertainty and sensitivity analysis of complex chemistry reaction sets [577].

\subsection{Modeling techniques}

Again, there are at least three regions of interest in modeling plasma-liquid interactions. The first addresses the gas phase properties-plasma generation, gas flow, plasma kinetics. The degree of sophistication of this modeling varies from global (0D) to multidimensional. Fortunately, there have been intensive investigations into the reaction mechanisms for humid air plasmas, or plasma jets consisting of rare gases flowing into humid air, and so there is a maturing baseline for the gas phase portion of the model. In principle, these gas phase models
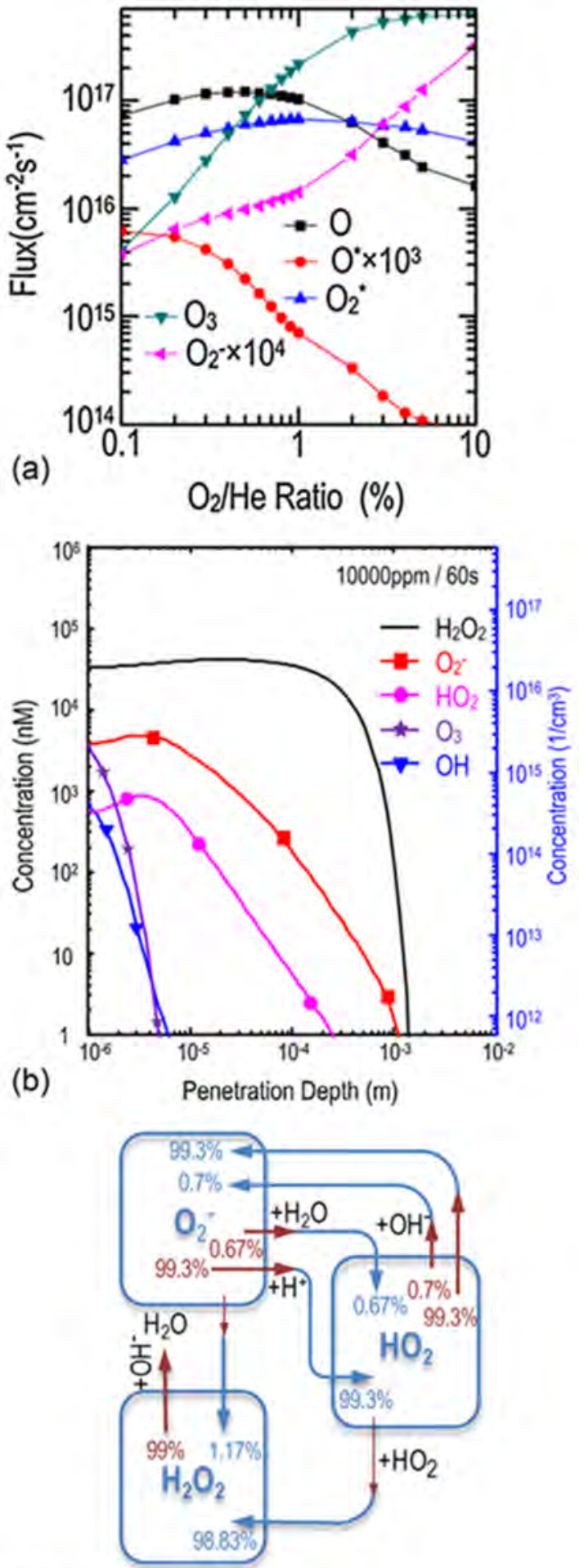

(c)

Penetration $=10 \mu \mathrm{m}$

Figure 27. Gas and liquid phase reactant densities from an atmospheric pressure plasma sustained in $\mathrm{He} / \mathrm{O}_{2}$ in contact with water. (a) Fluxes of ROS to the surface of the water for gas phase $\mathrm{O}_{2}$ concentrations of $0-10 \%$. (b) In liquid densities of ROS as a function of distance of penetration into the bulk liquid for $\mathrm{He} /$ $\mathrm{O}_{2}=99 / 1$. (c) The pathway chain map for key ROS is shown for $10 \mathrm{~mm}$ into the liquid. The percentage for each pathway indicates the contribution for generation or loss of the species. Reproduced with permission from [188], copyright 2014 Springer. 
are directly applicable to the investigation of plasma-liquid interaction given the proper boundary conditions between the plasma and the water.

The second region addresses transport and reactions within the liquid. Addressing transport within the liquid is problematic since there are multiple spatial scales that must be addressed. For example, a subset of the gas phase species transporting into the liquid have short reaction lengths, perhaps as small as a few microns, whereas the depth of the liquid may be hundreds of microns (liquids covering wounds) to tens of $\mathrm{cm}$. In principle, resolving this dynamic range is only a matter of computational resources. However, approximations may capture this surface-layer reactivity without actually resolving the spatial lengths. An even shorter spatial scale is that of the electrical double layer in the liquid and sheath in the gas phase [578]. The electrical double layer is conventionally thought of as forming at the surface of a metal electrode immersed in an ionic liquid. The thickness of the electrical double layer may be only $1 \mathrm{~nm}$. However, an electrical double layer will likely occur at the plasma liquid layer as well. The water will have a finite dielectric relaxation time and so have dielectric properties which produce some charging of the surface of the liquid or near the surface layer. The sheath on the gas plasma side of the plasma-liquid interface will bring a high potential and a space charge region to the proximity of the surface. These conditions are, in principle, the same as for an electrode in an ionic liquid, and so an electrical double layer will likely form. Coupling these scales in models will be challenging although considerable progress is being made [579].

The most basic approach to modeling the plasma-induced liquid phase chemistry is the well-stirred reactor approximation where convective mixing of the liquid is sufficiently rapid that reactants entering the liquid are fully mixed. Although computationally convenient, it may be difficult to properly represent the species in the thin reactive layer below the plasma liquid interface. The next more sophisticated approach is to spatially resolve transport and reactions within the liquid with transport being represented by diffusion. Since the Peclet number is typically large in liquids, a model in which transport is limited to diffusion is strictly only valid for conditions where diffusion should dominate, such as thin water layers. For thick water layers, advective motion of the liquid should be considered [323]. This motion can result from an external pressure gradient, as in conventional fluid flow, can be driven by plasma initiated and electrical forces, or by gas flow due to an impinging jet. In all cases, deformation of the liquid surface should be accounted for. A subset of models of the deformation of the plasma-liquid interface by the plasma is the case of simulating plasmas-in-bubbles. Here, there is tight coupling between the electrical forces and the shape of the bubble. Mass transport between the plasma and liquid is much more critical due to the large surfaceto-volume ratio.

The third region of interest is the interface between the gas phase and the liquid. The modeling technique used here depends on the physical models adopted. In the absence of an adsorbed layer, transport through the plasma-liquid interface between the gas phase and liquid phase depends primarily on the differences in densities between those phases, and the condition dependent transport probabilities. When using an adsorbed layer, the plasma liquid interface is represented by separate algorithms that account for the adsorbed density of species at the plasma liquid interface. In either case, the deformation of the surface of the liquid should be accounted for.

The sophistication of models of the gas phase and liquid plasmas then dictate the manner in which the electric field is derived. In spatially resolved models of the gas phase plasmas, the electric field is produced by either directly solving for the electric potential from Poisson's equation, or derived through current continuity and a quasi-neutrality approximation. The latter requires some form of ambipolar transport. In principle, the same techniques can be extended to the liquid phase. In the case of solving Poisson's equation, continuity equations are derived for charged particle transport in the liquid which includes contributions from drift in the electric field. A charge density is derived and this charge density is used to solve Poisson's equation. In this technique the appropriate polarizability of the constituents of the liquid need to be accounted for in order to provide the permittivity required to solve Poisson's equation. Although this technique may be computationally intensive, it is the least ambiguous. The alternative methods present challenges. In most alternative methods of gas phase plasmas, ambipolar enhanced transport coefficients are derived for the ions. Charge neutrality is then maintained by requiring that the density of electrons equals the charge density of the sum of all ions. This is not particularly ambiguous in the gas phase where one can be assured that electrons exist with some reasonable density in regions where the plasma is being actively produced, and that electrons are the most mobile species in the absence of externally applied magnetic fields. This is not the case in the liquid where electrons rapidly solvate and undergo reactions. There is no guarantee that the solvated electron will have either the highest mobility or an appreciable density throughout the liquid plasma. It is therefore unclear that a single species can be singled out (as electrons are in the gas phase) to be the charge balancing species. In the liquid, a fully self-consistent ambipolar transport algorithm would be required. This involves a matrix solution of the charge and current conserving transport coefficients whose solution provides the local electric field consistent with the quasi-neutral approximation.

The just described method presupposes that the plasmainduced charge density produces a large fraction of the ions in the liquid. In heavily buffered solutions or previously conductive solutions, the perturbation to the charge density by the plasma may not be large. In that case, the local electric fields can be produced by current continuity based on the initial conductivity of the buffered solution.

With all of these challenges, advances are required in modeling of plasma interactions with the liquid-gas interface. The interactions become more complex in the presence of fluid motion, and if the flow is turbulent, the variety of 
length and time scales means that simple descriptions may be inadequate in capturing the underlying physio-chemical phenomena [580, 581]. Another challenging dynamic to model, depending on the momentum of the gas and liquid phases, is the movement or displacement of the liquid-gas interface. In the simplest of cases-low Reynolds numbers and low rates of mass-transfer-a momentum or force balance can provide a relatively accurate prediction of the interface location, stationary or periodic. However, if the plasma stream or jet is turbulent, the location and displacement of the interface can be intermittent and difficult to both describe and predict [582].

Turbulence is typically not included in most plasma-liquid interaction models. Modeling the effects of turbulence requires the treatment of four phenomena: the effects of turbulence on the fluid (in each phase), on reactant transport, on the chemical reactions, and on the liquid-gas interface. The majority of turbulence models in use today are based on Reynolds-averaged Navier Stokes (RANS) formulations. RANS approaches have their limitations, for example, when periodic motion is present as may occur at the plasma-liquid interface [583]. In these cases, either direct numerical simulation (DNS) or large-eddy simulation (LES) may be appropriate for plasmas near the liquid-gas interface [584]. Turbulence has been shown to increase both large-scale mixing, via entrainment [585], and small-scale mixing, via vortex bending and stretching [586]. There are a number of techniques that model the effects of turbulence on chemical reactions $[587,588]$. One of the more general approaches is probability-based methods [589].

The transition between laminar and turbulent flow has been investigated extensively in thermal arcs, torches and thermal arcjets [590-592]. Sophisticated 3D models have been developed which combined computational fluid dynamics, electromagnetics, plasma-surface interactions and mixture-dependent thermodynamics [590, 591]. With the exception of the thermal arcs being well represented by local-thermodynamic-equilibrium, there are many similarities between the turbulence experienced in thermal arcs and those observed for non-equilibrium plasma jets.

Simulation of liquid-gas interfacial flows is a significant challenge due to the evolving, non-regular shapes and moving boundaries across which density changes by several orders of magnitude [593]. Both Lagrangian and Eulerian methods have been extensively used in the past for modeling multiphase interfacial flows [594]. Two representative methods are the volume of fluid (VOF) and level set (LS) approaches [595597]. The importance of small deformations (as electric field enhancing spots) and the required accuracy of the interface tracking might complicate the implementation. Current stateof-the-art turbulent flow simulations have significant difficulty resolving liquid gas interfacial dynamics. These interfaces are thin and rapidly evolve. The sequence of images in figure 28 shows the development of the Kelvin-Helmholtz instability along the interface of a temporal, planar shear layer. Temporal shear layers are regularly used to investigate turbulent flow phenomena as they contain many of the dynamics present in flows undergoing transition to turbulence while having a relatively small and compact computational domain [598].
The interaction of atmospheric pressure plasmas with evaporating liquids is not restricted to biological and environmental applications. There is a large knowledge base of such interactions in the context of arc welding of metals. In these applications, there is usually a pool of melted (liquid) metal at the intersection of the arc with the solid work piece which produces a pool of metal vapor which in turn evaporates into plasma. The metal vapor significantly alters the conductivity and transport coefficients of the arc. Sophisticated 3D models have been developed of these processes [599].

\section{Conclusions and roadmap}

A large variety of plasmas interacting with liquids have been investigated. There is surprisingly little knowledge available, particularly on reactive species densities, for direct discharges in liquids, discharge in bubbles and aerosol containing plasmas. A detailed framework of species transfer at the gas-liquid interface has been developed for investigation of aerosols that could be an excellent starting point to implement this method for plasmas interacting with liquids. Charged species transfer and photon-induced effects need to be included in this framework. The plasma community can benefit from established work in the field of electrochemistry, photolysis and advanced oxidation technology in this process.

We have reviewed the fluid dynamics, heat and mass transfer that occur during plasma-liquid interactions. We included discussion emphasizing the extremely dynamic plasma-liquid interface where Taylor cones, capillary waves and dimples may occur. Since the ratio of advective transport to diffusive transport is usually large in the bulk plasma, plasma-induced liquid convection plays an important role in the gas phase plasma reactive transfer to the liquid phase.

There are good starting points for developing kinetic models of the chemistry in water vapor containing plasmas. The experimental validation of current modeling efforts is limited and a critical review on the species transfer from the gas to liquid phase particularly for ions is needed.

Several data needs are highlighted in this manuscript including:

- Electrons colliding with relevant reactive species such as $\bullet \mathrm{OH}$ and $\mathrm{H}_{2} \mathrm{O}_{2}$

- New data and improvements to existing data for ion reactions.

- Temperature and pressure dependence of neutral reactions.

- Charged species transfer across the gas-liquid interface including electron-induced reactions and energy transfer.

- More extensive information on the $\mathrm{pH}$, temperature and electrical field effects on liquid phase reactions.

- Rates for heavy particle reactions with fast atoms and vibrationally excited $\mathrm{H}_{2} \mathrm{O}$ and ${ }^{\bullet} \mathrm{OH}$.

An impressive set of gas phase diagnostics are available that can measure many plasma parameters. Due to the presence of unknown water vapor gradients in many of the discharges of interest, many diagnostics such as LIF require detailed excited state lifetime measurements and collisional transfer models. 

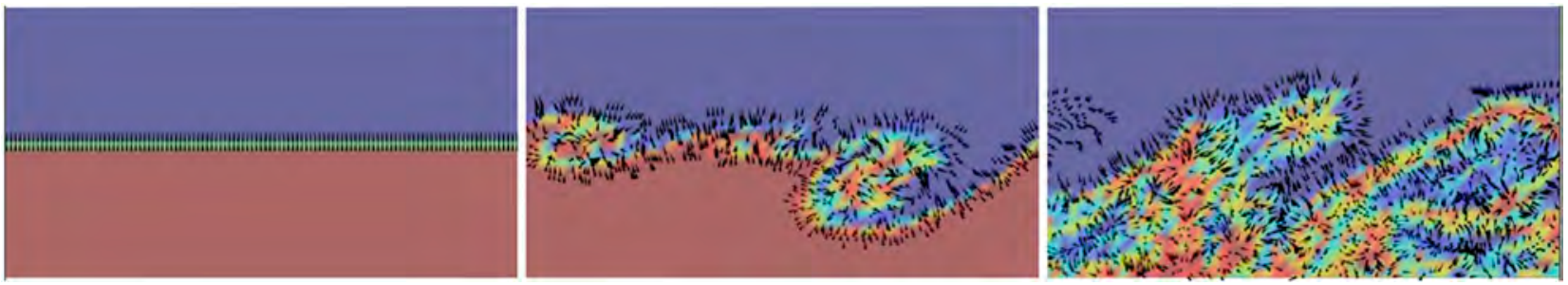

Figure 28. This sequence of images [600] shows the evolution of a multiphase temporal shear layer obtained from a model using a Lagrangian VOF approach [601]. Vectors point from the liquid into the gas and reflect instability the development of the Kelvin Helmholtz. While surface tension may stabilize the short wavelength instability, longer waveforms appear, stretch and distort the surface, creating new interface. Over the time interval shown vortices form and a transition to a fully turbulent interface occurs.

The interpretation of optical emission spectra remains a challenge due to complex kinetics, high collisionality and lack of some relevant cross sections. Direct discharges in liquids and discharges in bubbles are almost unexplored by advanced diagnostics due to their limited optical accessibility and partly stochastic nature.

The diagnostics of plasma-induced (often short-lived) reactive liquid phase chemistry are currently a great challenge because most available techniques are not selective or influence the plasma induced liquid phase chemistry. An initial set of reference analytical methods for commonly produced species have been proposed. However, further standardization of the liquid phase detection techniques for plasma induced liquid phase chemistry is urgently needed.

Surface processes have been extensively studied by diagnostics in the field of aerosols. However these diagnostics are usually performed under well-controlled conditions that do not occur in most plasma discharges. An extensive suite of diagnostics to assess macroscopic transport is available from different fields. A more extensive use of these techniques in the study of plasma-liquid interactions is important to further advance our understanding of plasma-induced liquid phase chemistry.

In spite of many challenges for modeling plasma-liquid interactions, significant progress has been made in recent years addressing the coupling of gas phase plasma kinetics with liquid phase reactivity. Direct liquid phase discharges remain a challenge and will require advances in our understanding of discharge ignition and liquid phase ionization processes in order to accurately model these processes. Models that couple laminar and turbulent gas flow, and multiphase interfacial flows have been developed for thermal plasmas. These approaches need to be extended to non-equilibrium atmospheric pressure plasmas.

In recent years, research on plasma-liquid interactions has been motivated by the growing field of plasma medicine which has led to the investigation of plasma jets and dielectric barrier discharges with liquids. These systems encompass highly dynamic and often stochastic-like behavior in space and time combined with complex flow patterns. Many research groups around the world have developed their own plasma sources. Establishing standards for a few reference plasma-liquid systems would foster exchange and comparison of data and so deepen our understanding of plasma-liquid interactions. In addition, the use of ionic liquids enables investigation of plasma-liquid interactions at reduced pressure for diffuse glow discharges without significant gas heating. This could provide an important simplified reference system for plasma-liquid interactions although not for aqueous solutions.

In conclusion, the complex and highly dynamic plasmaliquid interface provides a wealth of challenging, fundamental and interesting scientific questions. Addressing these challenges and questions would benefit from a coordinated effort to further develop and implement state-of-the-art diagnostics and modeling. These efforts will enable the plasma community to more rapidly exploit plasma-induced chemistry in liquids for emerging applications such as plasma medicine, material synthesis and environmental stewardship. The resulting increased understanding will lead to new applications and enable us to tackle many societal challenges related to energy, environment and health.

\section{Acknowledgments}

This manuscript originated from discussions at the Lorentz Center Workshop 'Gas/Plasma-Liquid Interface: Transport, Chemistry and Fundamental Data' that took place at the Lorentz Center, Leiden University in the Netherlands from August 4, through August 8, 2014, and follow-up discussions since the workshop. All authors acknowledge the support of the Lorentz Center, the COST action TD1208 (Electrical Discharges with Liquids for Future Applications) and the Royal Dutch Academy of Sciences for their financial support. PJB, MJK, DBG and JEF acknowledge the support of the 'Center on Control of Plasma Kinetics' of the United States Department of Energy Office of Fusion Energy Science (DE-SC0001319). In addition, PJB and BRL acknowledge the support of the National Science Foundation (PHY 1500135 and CBET 1236225, respectively). In addition the enormous help of Mrs. Victoria Piorek (University of Minnesota) in the formatting of the final document including the references is gratefully acknowledged. 
Appendix

Table A1. Overview of gas phase plasma diagnostic methods.

\begin{tabular}{|c|c|c|c|}
\hline Quantity & Method & Comment & Ref. \\
\hline$I, V$ & $\begin{array}{l}\text { Shunt resistor, Rogowski coil, voltage } \\
\text { dividers }\end{array}$ & & {$[602]$} \\
\hline \multirow[t]{3}{*}{$P$} & (a) Current-voltage & (a) Used for pulsed, DC and AC discharges & {$[602-606]$} \\
\hline & (b) Voltage-charge & (b) Typically used for DBD & \\
\hline & (c) Thermally based power meters & (c) Used for RF and MW discharges & \\
\hline \multirow[t]{4}{*}{$n_{\mathrm{e}}$} & (a) Stark broadening & (a) Typically $H_{\beta}, n_{\mathrm{e}}>10^{20} \mathrm{~m}^{-3}$ & {$[449,607-612]$} \\
\hline & (b) Thomson scattering & (b) In situ, calibration necessary & \\
\hline & (c) Interferometry & (c) Line of sight measurement, effect of $T_{\mathrm{g}}$ & \\
\hline & (d) Continuum radiation & (d) Absolute calibration necessary & \\
\hline \multirow[t]{3}{*}{$\overline{T_{\mathrm{e}}}$} & (a) Thomson scattering & (a) In situ (EEDF remains a challenge) & {$[608-610,612,613]$} \\
\hline & (b) Line intensity ratio & $\begin{array}{l}\text { (b) Need of collisional radiative model, reaction } \\
\text { rates only available for limited number of gas } \\
\text { mixtures }\end{array}$ & \\
\hline & (c) Continuum radiation & (c) Absolute calibration necessary & \\
\hline
\end{tabular}

\begin{tabular}{|c|c|}
\hline$T_{\mathrm{g}}$ & $\begin{array}{l}\text { (a) Rotational emission spectra } \\
\text { (b) Rotational absorption spectroscopy } \\
\text { (c) Rotational LIF spectra } \\
\text { (d) Rotational Raman Scattering } \\
\text { (e) van der Waals broadening } \\
\text { (f) Doppler broadening } \\
\text { (g) Rayleigh scattering } \\
\text { (h) Schlieren imaging } \\
\text { (i) Thermal probes }\end{array}$ \\
\hline
\end{tabular}

(a) Non-equilibrium effects possible

(b) Ground state diatomic molecule

(c) Ground state diatomic molecule

(d) Ground state diatomic molecule

(e) Atomic line with small Stark broadening at low gas temperatures

(f) Atomic line with small Stark broadening at elevated gas temperatures

(g) Gas density measurement, species dependent

(h) Refractive index measurement

(i) Possible interactions of plasmas with probe

\begin{tabular}{lll}
\hline$E / N$ & (a) $\mathrm{N}_{2}(C) / \mathrm{N}_{2}^{+}(B)$ ratio & (a) Electron excitation should be dominant \\
& (b) CARS & (b) Performed in $\mathrm{H}_{2}$ and $\mathrm{N}_{2}$ containing gases \\
\hline $\mathrm{H}_{2} \mathrm{O}$ & (a) Absorption & (a) Line integrated measurement \\
& (b) Raman scattering & (b) Spatially resolved but less sensitive \\
& (c) OH LIF (quenching measurement) & (c) Other gas molecules determine detection limit
\end{tabular}

\begin{tabular}{lll}
\hline $\mathrm{N}_{2}, \mathrm{O}_{2}$ & (a) Raman scattering & (a) Spatially resolved \\
& (b) Mass spectrometry & (b) Sampling only at solid interface
\end{tabular}

$\mathrm{O}^{\bullet}, \mathrm{N}^{\bullet}, \mathrm{H}^{\bullet}, \ldots \quad$ (a) TaLIF

(a) Requires calibration and quenching corrections

$[455,614]$
(b) VUV absorption
(c) Mass spectrometry

(b) Requires VUV wavelengths which are absorbed in $\mathrm{O}_{2}, \mathrm{H}_{2} \mathrm{O}$

(c) Sampling only at solid interface, interferes with cracking patterns from internal MS ionization source

\begin{tabular}{ll}
\hline $\mathrm{NO}, \bullet \mathrm{OH}$, & (a) LIF \\
& (b) UV absorption \\
& (c) Mass spectrometry
\end{tabular}

(a) Requires calibration

$[76,437,454,622]$

(c) Mass spectrometry

(b) Line integrated measurement

(c) Sampling only at solid interface, interferes with cracking patterns from internal MS ionization source

\begin{tabular}{lll}
\hline $\mathrm{Ar}_{m}, \mathrm{He}_{m}, \mathrm{~N}_{2}(A)$ & (a) LIF & (a) Requires calibration by e.g. Rayleigh \\
scattering
\end{tabular}

$[623-626]$

(b) Line integrated measurement

\begin{tabular}{lll}
\hline (H)NO $\mathrm{NO}_{x}^{(-)}, \mathrm{H}_{2} \mathrm{O}_{2}$ & Multi-pass absorption (FTIR) & Line integrated measurement \\
\hline Positive ions & $\begin{array}{l}\text { (a) Mass spectrometry } \\
\text { (b) LIF }\end{array}$ & (a) Sampling only at solid interface \\
\hline Negative ions & $\begin{array}{l}\text { (b) Only particular ions such as Ar }{ }^{+}, \mathrm{N}_{2}^{+} \text {have been studied } \\
\text { (b) Photo-detachment }\end{array}$ & $\begin{array}{l}\text { (a) Sampling only at solid interface } \\
\text { (b) Requires absolute determination of } n_{\mathrm{e}}\end{array}$
\end{tabular}

Note: This list is provided as a starting reference point and is not intended to be exhaustive. 
Table A2. Common plasma produced liquid phase species, their possible detection methods and scavengers with some recommendations.

\begin{tabular}{|c|c|c|c|c|}
\hline Species & Detection method & Scavengers & Comment & Ref. \\
\hline \multirow[t]{2}{*}{$\begin{array}{l}\text { Hydrogen } \\
\text { peroxide } \\
\left(\mathrm{H}_{2} \mathrm{O}_{2}\right)\end{array}$} & $\begin{array}{l}\text { (a) Colorimetric assay by titanyl sulfate reagent } \\
\text { producing the yellow product pertitanic acid, } \\
\text { absorption maxima at } 405-410 \mathrm{~nm}\end{array}$ & Catalase & $\begin{array}{l}\text { (a) Stable color complex, only known } \\
\text { interferences from } \mathrm{NO}_{2}^{-} \text {(elimination } \\
\text { by azide), linear response up to mM } \\
\text { concentration of } \mathrm{H}_{2} \mathrm{O}_{2}\end{array}$ & $\begin{array}{l}\text { (a) }[484,488 \text {, } \\
633]\end{array}$ \\
\hline & $\begin{array}{l}\text { (b) Colorimetric/fluorometric assay by } \\
\text { nonfluorescent reagent amplex red } \\
\text { (10-acetyl-3,7-dihydroxyphenoxazine) } \\
\text { producing the red-colored and fluorescent } \\
\text { product resorufin (excitation/emission } \\
\text { maxima } 571 / 585 \mathrm{~nm})\end{array}$ & & $\begin{array}{l}\text { (b) Possible interferences from thiols, } \\
\text { air sensitive, pH-dependent reaction, } \\
\text { high sensitivity to } \mathrm{H}_{2} \mathrm{O}_{2} \text {, low } \\
\text { detection limit }(\sim 10 \mathrm{pM}) \text {, linear } \\
\text { response up to } \mu \mathrm{M} \text { concentration of } \\
\mathrm{H}_{2} \mathrm{O}_{2}\end{array}$ & (b) $[634,635]$ \\
\hline
\end{tabular}

Hydroxyl (a) EPR spectroscopy by spin-trapping using radical $\left({ }^{\bullet} \mathrm{OH}\right)$ 5,5-dimethyl-1-pyrroline-N-oxide (DMPO) or 5-tert-butoxycarbonyl-5-methyl-1pyrroline-N-oxide (BMPO)

(b) HPLC analysis of hydroxylated product (fluorescent) of terephthalic acid TFA (nonfluorescent)

(c) LC/ESI-MS/MS analysis, by multiple reaction monitoring (MRM) mode, of the product of the reaction of ${ }^{\bullet} \mathrm{OH}$ with 5,5-dimethyl-1-pyrroline-N-oxide (DMPO) using N-methyl-2-pyrrolidone (NMP) as the internal standard

(d) Spectrophotometric or HPLC detection of formaldehyde, product of $\mathrm{tBuOH}$ reaction with ${ }^{\bullet} \mathrm{OH}$ or ion chromatographic detection of methanesulfinic acid, product of DMSO reaction with ${ }^{\bullet} \mathrm{OH}$ $t \mathrm{BuOH}$

a) Spin trap OH-product is not stable in time, online EPR detection in plasma system not possible. For DMPO, sum concentrations of ${ }^{\bullet} \mathrm{OH}+\mathrm{O}_{2}^{\bullet-}$ can only be detected

(b) Can be used directly in plasma treated liquid, measurements with several different concentrations of TFA recommended

(c) $t \mathrm{BuOH}$ is reported to be the only aliphatic alcohol that does not promote the formation of $\mathrm{O}_{2}^{--}$and so of ${ }^{\bullet} \mathrm{OH}$ (a) [482]

(b) [489]

(d) $[636,637]$
Superoxide anion radical $\left(\mathrm{O}_{2}^{\cdot-}\right)$ (a) EPR spectroscopy by spin-trapping using 5,5-dimethyl-1-pyrroline-N-oxide (DMPO) or 5-tert-butoxycarbonyl-5-methyl-1pyrroline-N-oxide (BMPO)

(b) Spectrophotometric detection of nitroform anion $\left(\mathrm{NF}^{-}\right)$formed by reduction of tetranitromethane (TNM), absorption maxima at $350 \mathrm{~nm}$

Ozone $\left(\mathrm{O}_{3}\right)$

(a) Spectrophotometric detection of decolorozation of potassium indigo trisulfonate dye by ozone, absorption detection at $600 \mathrm{~nm}$

(b) DCF-derived fluorescent probe: 6-(But-3-en1-yloxy)-2,7-dichloro-9-(2-(hydroxymethyl) phenyl)-3H-xanthen-3-one. Excitation 497, fluorescence $523 \mathrm{~nm}$

\section{SOD}

(a) By DMPO, sum concentrations of $\bullet \mathrm{OH}+\mathrm{O}_{2}^{\bullet-}$ can only be detected Spin trap $\mathrm{O}_{2}^{\cdot-}$ product is not stable in time, online EPR detection in plasma system not possible

(b) Reacts with slower rate with $\mathrm{H}^{\bullet}$ radical, other products of TNM conversion may interfere $\left({ }^{\bullet} \mathrm{NO}_{2}\right.$, $\mathrm{NO}_{2}^{-}$), $\mathrm{NF}^{-}$reacts with ${ }^{\bullet} \mathrm{OH}$
Nitrite ions, azide (a) Sensitive but very demanding method for proper handling of liquid samples, analytical problems due to low stability of ozone in water (fast decay), indigo blue color bleaching also possible by other oxidative agents $\left({ }^{\bullet} \mathrm{OH}, \mathrm{H}_{2} \mathrm{O}_{2}\right)$

(b) $5<\mathrm{pH}<10$. A second step after ozone exposure is needed in order to obtain the fluorescent product. Selective against hydroxyl radical, singlet oxygen, superoxide, hydrogen peroxide, hypochlorite. The probe is destroyed in hydrogen peroxide under basic conditions $(\mathrm{pH} \geq 10$ )

\footnotetext{
Nitrite $\left(\mathrm{NO}_{2}^{-}\right) \quad$ (a) Spectrophotometric detection of Griess diazotization reaction product of nitrite with sulfanilic acid and $N$-(1-naphthyl)-ethylene diamine hydrochloride (pink colored azo dye), absorption detection at $525 \mathrm{~nm}$
}

(b) Ion chromatography
Azide
under
acidic
(a) Interferences with $\mathrm{H}_{2} \mathrm{O}_{2}$ during diazotization reaction (acidic conditions)

conditions (a) $[482]$

(b) $[638]$

(a) $[79,638]$

(b) [639]

(a) $[486,633]$ 
Table A2. (Continued)

\begin{tabular}{|c|c|c|c|c|}
\hline Species & Detection method & Scavengers & Comment & Ref. \\
\hline Nitrate $\left(\mathrm{NO}_{3}^{-}\right)$ & $\begin{array}{l}\text { (a) Spectrophotometric detection of reaction } \\
\text { product with 2,6-dimethylpehenol to 4-nitro- } \\
2,6 \text {-dimethylphenol, absorption detection at } \\
340 \mathrm{~nm} \text {; } \\
\text { (b) Ion chromatography }\end{array}$ & & & {$[503,633]$} \\
\hline \multirow[t]{2}{*}{$\begin{array}{l}\text { Peroxynitrite } \\
\left(\mathrm{ONOO}^{-}\right)\end{array}$} & $\begin{array}{l}\text { (a) Colorimetric/fluorometric } \\
\text { assay with nonfluorescent } \\
\text { 2,7-dichlorodihydrofluorescein }\left(\mathrm{DCFH}_{2}\right) \\
\text { producing with } \mathrm{ONOO}^{-} \text {fluorescent product } \\
\text { dichlorofluorescein }(\mathrm{DCF}) \text {, absorption } \\
\text { detection at } 500 \mathrm{~nm} \text {, fluorescence detection } \\
\text { at } 502 \mathrm{~nm} \text { (excitation) and } 523 \mathrm{~nm} \text { (emission) }\end{array}$ & \multirow[t]{2}{*}{ Uric acid } & 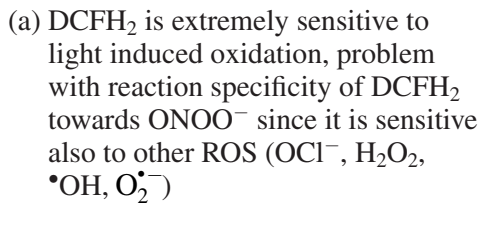 & (a) $[487,503]$ \\
\hline & $\begin{array}{l}\text { (b) Indirect determination through kinetic } \\
\text { analysis of third-order reaction between } \\
\mathrm{HNO}_{2} \text { and } \mathrm{H}_{2} \mathrm{O}_{2}\end{array}$ & & $\begin{array}{l}\text { b) Evaluation of ONOOH from } \\
\text { concentration decay of } \mathrm{NO}_{2}^{-} \text {and } \\
\mathrm{H}_{2} \mathrm{O}_{2} \text { in plasma treated liquid with } \\
\text { constant (buffered) pH }\end{array}$ & (b) $[488]$ \\
\hline \multirow[t]{2}{*}{$\begin{array}{l}\text { Singlet } \\
\text { Oxygen }\left({ }^{1} \mathrm{O}_{2}\right)\end{array}$} & (a) NIR phosphorescence at $1275 \mathrm{~nm}$ & \multirow{2}{*}{$\begin{array}{l}\text { Sodium } \\
\text { azide, } \\
\text { histidine }\end{array}$} & $\begin{array}{l}\text { (a) Weak emission due to spin } \\
\text { restrictions }\end{array}$ & (a) $[640]$ \\
\hline & (b) Singlet oxygen sensor green & & $\begin{array}{l}\text { (b) The probe itself can act as a } \\
\text { photosensitizer for singlet oxygen } \\
\text { production and it degrades in UV } \\
\text { producing ROS }\end{array}$ & (b) $[641]$ \\
\hline
\end{tabular}

\section{References}

[1] Cavendish H 1785 Experiments on air Phil. Trans. $75372-84$

[2] Gubkin J 1887 Elektrolytische Metallabscheidung an der freien Oberfläche einer Salzlösung Ann. Phys. 32114

[3] Hickling A and Ingram M D 1964 Contact glow-discharge electrolysis Trans. Faraday Soc. 60 783-93

[4] Martin J C 1996 J.C. Martin on Pulsed Power (New York: Plenum)

[5] Sato M, Ohgiyama T and Clements J S 1996 Formation of chemical species and their effects on microorganisms using a pulsed high-voltage discharge in water IEEE Trans. Ind. Appl. 32 106-12

[6] Foster J E, Sommers B S, Gucker S N, Blankson I M and Adamovsky G 2012 Perspectives on the interaction of plasmas with liquid water for water purification IEEE Trans. Plasma Sci. 40 1311-23

[7] Sunka P 1999 Generation of chemically active species by electrical discharges in water Plasma Source Sci. Technol. 8 258-65

[8] Webb M R and Hieftje G M 2009 Spectrochemical analysis by using discharge devices with solution electrodes Anal. Chem. 81 862-7

[9] Rosen A L and Hieftje G M 2004 Inductively coupled plasma mass spectrometry and electrospray mass spectrometry for speciation analysis: applications and instrumentation Spectrochim. Acta B 59 135-46

[10] Smoluch M, Mielczarek P and Silberring J 2016 Plasma-based ambient ionization mass spectrometry in bionanalytical sciences Mass Spectrom. Rev. 35 22-34

[11] Mariotti D, Patel J, Svrcek V and Maguire P 2012 Plasma-liquid interactions at atmospheric pressure for nanomaterials synthesis and surface engineering Plasma Proc. Polym. 9 1074-85

[12] Ishijima T, Nosaka K, Tanaka Y, Uesugi Y, Goto Y and Horibe H 2013 A high-speed photoresist removal process using mutibubble microwave plasma under a mixture of multiphase plasma environment Appl. Phys. Lett. 103142101

[13] Friedrich J F, Mix N, Schulze R-D, Meyer-Plath A, Ranjit Joshi R and Wettmarshausen S 2008 New plasma techniques for polymer surface modification with monotype functional groups Plasma Proc. Polym. 5 407-23

[14] Bruggeman P J and Locke B R 2013 Low Temperature Plasma Technology ed P K Chu and X P Lu (London: Taylor and Francis)

[15] Fridman G, Friedman G, Gutsol A, Shekhter A B, Vasilets V N and Fridman A 2008 Applied plasma medicine Plasma Proc. Polym. 5 503-33

[16] Locke B R, Sato M, Sunka P, Hofman M R and Chang J S 2006 Electrohydrolic discharge and nonthermal plasma for water treatment Ind. Eng. Chem. Res. 45 882-905

[17] Bruggeman P J and Leys C 2009 Non-thermal plasmas in and in contact with liquids J. Phys. D: Appl. Phys. 42053001

[18] Elford M T 1995 The ratio $D T / \mu$ for electrons in water vapour at 294 K Aust. J. Phys. 48 427-37

[19] Barnett R N, Giniger R, Cheshnovsky O and Landman U 2011 Dielectron attachment and hydrogen evolution reaction in water clusters J. Phys. Chem. A 115 7378-91

[20] Ruiz-Vargas G and De Urquijo J 2009 Pressure-dependent electron attachment in $\mathrm{H} 2 \mathrm{O}$-air mixtures XXIX Int. Conf. Phenomena in Ionized Gases (Cancun, Mexico) pp PB1-7

[21] An W, Baumung K and Bluhm H 2007 Underwater streamer propagation analyzed from detailed measurements of pressure release J. Appl. Phys. 101053302

[22] Bonifaci N, Denat A and Atrazhev V 1997 Ionization phenomena in high-density gaseous and liquid argon in corona discharge experiments J Phys. D Appl. Phys. 302717

[23] Starikovskiy A, Yang Y, Cho Y I and Fridman A 2011 Non-equilibrium plasma in liquid water: dynamics of generation and quenching Plasma Source Sci. Technol. 20024003

[24] Samukawa S et al 2012 The 2012 plasma roadmap J. Phys. D: Appl. Phys. 45 253001-37

[25] Brenner M P, Hilgenfeldt S and Lohse D 2002 Single-bubble sonoluminescence Rev. Mod. Phys. 47 425-84

[26] De Giacomo A, Dell'Aglio M, De Pascale O and Capitelli M 2007 From single pulse to double pulse ns-laser induced breakdown spectroscopy under water: elemental analysis of aqueous solutions and submerged solid samples Spectrochim. Acta B 62 721-38 
[27] Chang J S, Urashima K and Uchida Y 2002 Characteristics of pulsed arc electrohydraulic discharges and their application to water treatment Res. Rep. Tokyo Denki Univ. 50 1-12

[28] Korobeinikov S, Melekhov A V and Besov A S 2002 Breakdown initiation in water with the aid of bubbles High Temp. 40 652-9

[29] Schoenbach K, Kolb J, Xiao S, Katsuki S, Minamitani Y and Joshi R 2008 Electrical breakdown of water in microgaps Plasma Source Sci. Technol. 17024010

[30] Pekker M and Shneider M N 2015 Pre-breakdown cavitation nanopores in the dielectric fluid in the inhomogeneous, pulsed electric fields J. Phys. D: Appl. Phys. $\mathbf{4 8} 424009$

[31] Marinov I, Starikowskaia S M and Rousseau A 2014 Dynamics of plasma evolution in a nanosecond underwater discharge J. Phys. D: Appl. Phys. 47224017

[32] Sarkisov G S, Zameroski N D and Woodworth J R 2006 Observation of electric field enhancement in a water streamer using kerr effect J. Appl. Phys. 99083304

[33] Lisitsyn I V, Nomiyama H, Katuski S and Akiyma H 1999 Thermal processes in a streamer discharge in water IEEE Trans. Dielectr. Electr. Insul. 6351

[34] Qian J et al 2005 Microbubble based model analysis of liquid breakdown initiation by a submicrosecond pulse J. Appl. Phys. 97113304

[35] Sommers B S and Foster J E 2014 Plasma formation in underwater gas bubbles Plasma Source Sci. Technol. 23015020

[36] Mededovic S and Locke B R 2007 Primary chemical reactions in pulsed electrical discharge channels in water J. Phys. D: Appl. Phys. 40 7734-46

[37] Franclemont J, Fan X and Mededovic Thagard S 2015 Physicochemical mechanisms of plasma-liquid interactions within plasma channels in liquid J. Phys. D: Appl. Phys. 48424004

[38] Locke B R and Thagard S M 2012 Analysis and review of chemical reactions and transport processes in pulsed electrical discharge plasma formed directly in liquid water Plasma Chem. Plasma Process. 32 875-917

[39] Baba K, Kaneko T and Hatakeyama R 2007 Ion irradiation effects on ionic liquids interfaced with RF discharge plasmas Appl. Phys. Lett. 90201501

[40] Meiss S A, Rohnke M, Kienle L, El Abedin S Z, Endres F and Janek J 2007 Employing plasmas as gaseous electrodes at the free surface of ionic liquids: deposition of nanocrystalline silver particles Chem. Phys. Chem. 8 50-3

[41] Becker K H, Schoenbach K H and Eden J G 2006 Microplasmas and applications J. Phys. D: Appl. Phys. 39 R55-70

[42] Kogelschatz U 2003 Dielectric-barrier discharges: their history, discharge physics and industrial applications Plasma Chem. Plasma Process. 23 1-46

[43] Fridman A 2008 Plasma Chemistry (Cambridge: Cambridge University Press)

[44] Tendero C, Tixier C, Tristant P, Desmaison J and Leprince P 2006 Atmospheric pressure plasmas: a review Spectrochim. Acta B $612-30$

[45] Lu X, Laroussi M and Puech V 2012 On atmospheric-pressure non-equilibrium plasma jets and plasma bullets Plasma Source Sci. Technol. 21034005

[46] Mezei P and Cserfalvi T 2007 Electrolyte cathode atmospheric glow discharges for direct solution analysis Appl. Spectrosc. Rev. 42 573-604

[47] Sen Gupta S K 2015 Contact glow discharge electrolysis: its origin, plasma diagnostics and non-faradaic chemical effects Plasma Source Sci. Technol. 24063001

[48] Andre P, Barinov Y, Faure G, Kaplan V, Lefort A, Shkol'nik S and Vacher D 2001 Experimental study of discharge with liquid non-metallic (tap-water) electrodes in air at atmospheric pressure J. Phys. D: Appl. Phys. 34 3456-65
[49] Machala Z, Jedlovsky I and Martišovitš V 2008 DC discharges in atmospheric air and their transitions IEEE Trans. Plasma Sci. 36 918-9

[50] Bruggeman P J, Ribezl E, Maslani A, Degroote J, Malesevic A, Rego R, Vierendeels J and Leys C 2008 Characteristics of atmospheric pressure air discharges with a liquid cathode and a metal anode Plasma Source Sci. Technol. 17025012

[51] Akishev Y, Aponin G, Grushin M, Karalnik V, Petryakov A and Trushkin N 2008 Self-running low-frequency pulsed regime of DC electric discharge in gas bubble immersed in a liquid J. Optoelectr. Adv. Maert. 10 1917-21

[52] Titov V, Rybkin V, Smirnov S, Kulentsan A and Choi H 2006 Experimental and theoretical studies on the characteristics of atmospheric pressure glow discharge with liquid cathode Plasma Chem. Plasma Process. 26 543-55

[53] Verreycken T, Bruggeman P and Leys C 2009 Anode pattern formation in atmospheric pressure air glow discharges with water anode J. Appl. Phys. 105083312

[54] Benilov M S and Kogelschatz U 2014 CLuster issue 'spots and patterns on electrodes of gas discharges' Plasma Source Sci. Technol. 23050201

[55] Akishev Y, Volchek A, Napartovich A and Trushkin N 1992 Near-anode instability of gas flow glow discharge Plasma Sources Sci. Technol. 1 190-94

[56] Shirai N, Uchida S and Tochikubo F 2014 Influence of oxygen gas on characteristics of self-organized luminous pattern formation observed in an atmospheric dc glow discharge using a liquid electrode Plasma Source Sci. Technol. 23054010

[57] Bobkova E, Smirnov S, Zalipaeva Y and Rybkin V 2014 Modeling chemical composition for an atmospheric pressure DC discharge in air with water cathode by $0 \mathrm{D}$ model Plasma Chem. Plasma Process. 34 721-43

[58] Bruggeman P J, Liu J J, Degroote J, Kong M G, Vierendeels J and Leys C 2008 DC excited glow discharges in atmospheric pressure air in pin-to-water electrode systems J. Phys. D: Appl. Phys. 41215201

[59] Xiong Q, Yang Z and Bruggeman P J 2015 Absolute OH density measurements in an atmospheric pressure DC glow discharge with water electrode by broadband UV absorption spectroscopy J. Phys. D: Appl. Phys. 48424008

[60] Naidis G V 2014 Production of active species in cold helium-air plasma jets Plasma Source Sci. Technol. 23065014

[61] Norberg S A, Tian W, Johnsen E and Kushner M J 2014 Atmospheric pressure plasma jets interacting with liquid covered tissue: touching and not-touching the liquid J. Phys. D: Appl. Phys. 4747503

[62] Tian W and Kushner M J 2014 Atmospheric pressure dielectric barrier discharges interacting with liquid covered tissue J. Phys. D: Appl. Phys. 47165201

[63] Kawamoto H and Umezu S 2005 Electrohydrodynamic deformation of water surface in a mteal pin to water plate corona discharge system J. Phys. D: Appl. Phys. 38 887-94

[64] Rumeli A and Hizal M 1986 Calculation of impulse flashover voltage of a water column IEEE Trans. Electr. Insul. 21 591-8

[65] Matsuo H, Fujishima T, Yamashita T and Takenouchi O 1996 Propagation velocity and photoemission intensity of a local discharge on an electrolytic surface IEEE Trans. Dielectr. Electr. Insul. 3 444-9

[66] Petrishchev V, Leonov S and Adamovich I V 2014 Studies of nanosecond pulse surface ionization wave discharges over solid and liquid dielectric surfaces Plasma Source Sci. Technol. 23065022

[67] Winters C, Petrishchev V, Yin Z, Lempert W R and Adamovich I R 2015 Surface charge dynamics and $\mathrm{OH}$ and $\mathrm{H}$ number density distributions in near-surface nanosecond 
pulse discharges at a liquid/vapor interface J. Phys. D Appl. Phys. 48424002

[68] Bruggeman P J, Degroote J, Vierendeels J and Leys C 2008 DC-excited discharges in vapour bubbles in capillaries Plasma Source Sci. Technol. 17025008

[69] Tachibana K, Takekata Y, Mizumoto Y, Motomura H and Jinno M 2011 Analysis of a pulsed discharge within single bubbles in water under synchronized conditions Plasma Source Sci. Technol. 20034005

[70] Iza F, Kim G J, Lee S M, Walsh J L, Zhang Y T and Kong M G 2008 Microplasmas: sources, particle kinetics, and biomedical applications Plasma Proc. Polym. 5 322-44

[71] Foest R, Kindel E, Ohl A, Stieber M and Weltmann K D 2005 Non-thermal atmospheric pressure discharges for surface modification Plasma Phys. Control. Fusion 47 B525-36

[72] Algwari Q T and O'Connell D 2011 Electron dynamics and plasma jet formation in a helium atmospheric pressure dielectric barrier discharge jet Appl. Phys. Lett. 99121501

[73] Stoffels E, Flikweert A J, Stoffels W W and Kroesen G M W 2002 Plasma needle: a non-destructive atmospheric plasma source for fine surface treatment of (bio)materials Plasma Source Sci. Technol. 11 383-8

[74] Kolb J F, Mohamed A A H, Price R O, Swanson R J, Bowman A, Chiavarini R L, Stacey M and Schoenbach K H 2008 Cold atmospheric pressure air plasma jet for medical applications Appl. Phys. Lett. 92241501

[75] Walsh J L and Kong M G 2008 Contrasting characterisitcs of linear and cross-field atmospheric pressure plasma jets Appl. Phys. Lett. 93111501

[76] van Ham B T J, Hofmann S, Brandenburg R and Bruggeman P J 2014 In situ absolute air, $\mathrm{O}_{3}$, and NO densities in the effluent of a cold RF argon atmospheric pressure plasma jet obtained by molecular beam mass spectrometry J. Phys. D: Appl. Phys. 47224013

[77] Wende $\mathrm{K}$ et al 2015 Identification of the biologically active liquid chemistry induced by a non-thermal atmospheric pressure plasma jet Biointerphases 10029518

[78] Parvulescu V I, Magureanu M and Lukes P 2012 Plasma Chemistry and Catalysis in Gases and Liquids (Germany: Wiley-VCH)

[79] Pavlovich M J, Chang H W, Sakiyama Y, Clark D S and Graves D B 2013 Ozone correlates with antibacterial effects from indirect air dielectric barrier discharge treatment of water J. Phys. D: Appl. Phys. 46145202

[80] Kahen K, Jorabchi K, Gray C and Montaser A 2004 Spatial mapping of droplet velocity and size for direct and indirect nebulization in plasma spectrometry Anal. Chem. 767194

[81] Olesik J W and Hobbs S E 1994 Monodisperse dried microparticulate injector: a new tool for studying fundamental processes in inductively coupled plasmas Anal. Chem. 663371

[82] Chan G-Y, Zhu Z and Hieftje G M 2012 Effect of single aerosol droplets on plasma impedance in the inductively coupled plasma Spectrochim. Acta B 7687

[83] Olesik J W and Kinzer J A 2006 Measurement of monodisperse droplet desolvation in an inductively coupled plasma using droplet size dependent peaks in Mie scattering intensity Spectrochim. Acta A 61696

[84] Shan Y and Mostaghimi J 2003 Numerical simulation of aerosol droplets desolvation in a radio frequency inductively coupled plasma Spectrochim. Acta B 581959

[85] Benson C M, Zhong J, Gimelshein S F, Levin D A and Montasera A 2003 Simulation of droplet heating and desolvation in inductively coupled plasma-part II: coalescence in the plasma Spectrochim. Acta B $\mathbf{5 8} 1453$

[86] Damiani D, Tarlet D and Meillot E 2014 A particle-trackingvelocimetry (PTV) investigation of liquid injection in a DC plasma jet J. Therm. Spray Technol. 23340
[87] Muboyadzhyan S A 2008 Deposition from the two-phase multicomponent flow of a vacuum-arc plasma containing droplets of an evaporated material Russ. Metall. 2008 112-21

[88] Shan Y and Hu Y 2012 Heat and mass transfer within an evaporating solution droplet in a plasma jet J. Therm. Spray Technol. 21676

[89] Marchand C, Chazelas C, Mariaux G and Vardelle A 2007 Liquid precursor plasma spraying: modeling the interactions between the transient plasma jet and the droplets J. Therm. Spray Technol. 16705

[90] Fauchais P, Joulia A, Goutier S, Chazelas C, Vardelle M, Vardelle A and Rossignol S 2013 Suspension and solution plasma spraying J. Phys. D: Appl. Phys. 46224015

[91] Fanelli F, Mastrangelo A M and Fracassi F 2014 Aerosolassisted atmospheric cold plasma deposition and characterization of superhydrophobic organic-inorganic nanocomposite thin films Langmuir 30857

[92] Fanelli F and Fracassi F 2014 Aerosol-assisted atmospheric pressure cold plasma deposition of organic-inorganic nanocomposite coatings Plasma Chem. Plasma Process. 34473

[93] Da Ponte G, Sardella E, Fanelli F, d'Agostino R, Gristina R and Favia P 2012 Plasma deposition of PEO-like coatings with aerosol-assisted dielectric barrier discharges Plasma Proc. Polym. 91176

[94] Da Ponte G, Sardella E, Fanelli F, Van Hoeck A, d'Agostino R, Paulussen S and Favia P 2011 Atmospheric pressure plasma deposition of organic films of biomedical interest Surf. Coat. Technol. 205 S525

[95] Ogawa D, Saraf I, Sra A, Timmons R, Goeckner M and Overzet L 2009 The direct injection of liquid droplets into low pressure plasmas J. Vac. Sci. Technol. A 27342

[96] Ogawa D, Chung C W, Goeckner M and Overzet L 2010 Transient effects caused by pulsed gas and liquid injections into low pressure plasmas Plasma Source Sci. Technol. 19034013

[97] Stallard C P, Iqbal M M, Turner M M and Dowling D P 2013 Investigation of the formation mechanism of aligned nano-structured siloxane coatings deposited using an atmospheric plasma jet Plasma Proc. Polym. 10888

[98] Zeleny J 1914 The electrical discharge from liquid points, and a hydrostatic method of measuring the electric intensity at their surfaces Phys. Rev. E 3 69-91

[99] Cloupeau M and Prunet-Foch B 1989 Electrostatic spraying of liquids in cone-jet mode J. Electrost. 22 135-59

[100] Cloupeau M and Prunet-Foch B 1994 Electrohydrodynamic spraying functioning modes: a critical review J. Aerosol Sci. 25 1143-57

[101] Bailey A G 1988 Electrostatic Spraying of Liquids (New York: Wiley)

[102] Ebeling D D, Westphall M S, Scalf M and Smith L M 2000 Corona discharge in charge reduction electrospray mass spectrometry Anal. Chem. 72 5158-61

[103] Xia Y and Cooks R G 2010 Plasma induced oxidative cleavage of disulfide bonds in polypeptides during nanoelectrospray ionization Anal. Chem. 82 2856-64

[104] Jaworek A and Krupa A 1997 Studies of the corona discharge in EHD spraying J. Electrost. 40-1 173-8

[105] Kim H H, Kim J H and Ogata A 2011 Time-resolved highspeed camera observation of electrospray J. Aerosol Sci. 42 249-63

[106] Pongrac B, Kim H H, Janda M, Martisovits V and Machala Z 2014 Fast imaging of intermittent electrospraying of water with positive corona discharge J. Phys. D: Appl. Phys. 47315202

[107] Burlica R, Shih K Y and Locke B R 2010 Formation of $\mathrm{H}_{2}$ and $\mathrm{H}_{2} \mathrm{O}_{2}$ in a water-spray gliding arc nonthermal plasma reactor Ind. Eng. Chem. Res. $496342-9$ 
[108] Maguire P D et al 2015 Controlled microdroplet transport in an atmospheric pressure microplasma Appl. Phys. Lett. 106224101

[109] Iqbal M M, Stallard C P, Dowling D P and Turner M M 2015 Three-dimensional coupled fluid-droplet model for atmospheric pressure plasmas Plasma Process. Polym. 12 201-13

[110] Iqbal M M and Turner M M 2015 Investigations of dropletplasma interaction using multi-dimensional coupled model Contrib. Plasma Phys. 55 627-42

[111] Kurahasi M, Katusura S and Mizuno A 1997 Radical formation due to discharge inside bubble in liquid J. Electrost. 4293

[112] Kitano K, Aoki H and Hamaguchi S 2006 Radio frequency driven atmospheric pressure plasmas in contact with liquid water Japan. J. Appl. Phys. 458294

[113] Aoki H, Kitano K and Hamaguchi S 2008 Plasma generation inside externally supplied Ar bubbles in water Plasma Source Sci. Technol. 17025006

[114] Tian W, Tachibana K and Kushner M J 2014 Plasmas sustained in bubbles in water: optical emission and excitation mechanisms J. Phys. D: Appl. Phys. 47055202

[115] Bruggeman P J, Verreycken T, Gonzalez M A, Walsh J L, Kong M G, Leys C and Schram D 2010 Optical emission spectroscopy as a diagnostic for plasmas in liquids: opportunities and pitfalls J. Phys. D: Appl. Phys. 43 124005-13

[116] Saitta A M, Saija F and Giaquinta P V 2012 Ab initio molecular dynamics study of dissociation of water under an electric field Phys. Rev. Lett. 108207801

[117] Gucker S N, Sommerer T J and Foster J E 2014 Plasma production in isolated bubbles IEEE Trans. Plasma Sci. 42 2636-7

[118] Pawłat J, Hensel K and Ihara S 2005 Decomposition of humic acid and methylene blue by electric discharge in foam Acta Phys. Slovaca 55 479-85

[119] Pawłat J, Hensel K and Ihara S 2006 Generation of oxidants and removal of indigo blue by pulsed power in bubbling and foaming systems Czech. J. Phys. 56 B1174-B8

[120] Hiller R A, Putterman S J and Weninger K R 1998 Timeresolved spectra of sonoluminescence Phys. Rev. Lett. 801090

[121] Gompf B, Gunther R, Nick G, Pecha R and Eisenmenger W 1997 Resolving sonoluminescence pulse width with timecorrelated single photon counting Phys. Rev. Lett. 791405

[122] Pecha R, Gompf B, Nick G, Wang Q and Eisenmenger W 1998 Resolving the sonoluminescence pulse shape with a streak camera Phys. Rev. Lett. 81 717-20

[123] Lohse D 2005 Sonoluminescence: cavitation hots up Nature $43433-4$

[124] Young F R 2004 Sonoluminescence (Boca Raton, FL: CRC)

[125] Dickenson E 1992 Introduction to Food Colloids (Oxford: Oxford Univeristy Press)

[126] Patino J, Delgado M and Fernandez J 1995 Stability and mechanical strength of aqueous foams containing food proteins Colloids Surf. A 99 65-78

[127] Kovscek A, Patzek T and Radke C J 1995 A mechanistic population balance model for transient and steady-state foam flow in boise sandstone Chem. Eng. Sci. 50 3783-99

[128] Pawłat J, Ihara S, Yamabe C and Pollo I 2005 Oxidant formation and the decomposition of organic compounds in foaming systems Plasma Proc. Polym. 2 218-21

[129] Bistron S, Sarre P and Szymonik B 1978 New method of conducting technological processes in dynamic foam (in Polish) Chemik 3 81-3

[130] Akishev Y, Arefi-Khonsari F, Demir A, Grushin M, Karalnik V, Petryakov A and Trushkin N 2015 The interaction of positive streamers with bubbles floating on a liquid surface Plasma Source Sci. Technol. 24065021
[131] Hussein R O, Nie X, Northwood D O, Yerokhin A and Matthews A 2010 Spectroscopic study of electrolytic plasma and discharging behaviour during the plasma electrolytic oxidation (PEO) process J. Phys. D: Appl. Phys. 43105203

[132] Stalder K R, Woloszko J, Brown I G and Smith C D 2001 Repetitive plasma discharges in saline solutions Appl. Phys. Lett. 79 4503-5

[133] Wehnelt A 1899 Ein elektrolytischer stromunterbrecher Ann. Phys. Chem. 304 233-72

[134] DeBaerdemaeker F, Simek M, Schmidt J and Leys C 2007 Characteristics of ac capillary discharge produced in electrically conductive water solution Plasma Source Sci. Technol. 16 341-54

[135] Maximov A I 2007 Physics, chemistry and applications of the AC diaphragm discharge and related discharges in electrolyte solutions Contrib. Plasma Phys. 47 111-8

[136] Prochazkova J, Stara Z and Krcma F 2006 Optical emission spectroscopy of diaphragm discharge in water solutions Czech. J. Phys. 56 B1314-9

[137] Dunleavy C S, Golosnoy I O, Curran J A and Clyne T W 2009 Characterisation of discharge events during plasma electrolytic oxidation Surf. Coat. Technol. $2033410-9$

[138] Krasik Y E, Grinenko A, Sayapin A, Efimov S, Fedotov A, Gurovich V T and Oreshkin V I 2008 Underwater electrical wire explosion and its applications IEEE Trans. Plasma Sci. 36 423-34

[139] Poschl U and Shiraiwa M 2015 Multiphase chemistry at the atmosphere-biosphere interface influencing climate and public health in the anthropocene Chem. Rev. 115 4440-75

[140] Seinfeld J H and Pandis S N 2006 Atmospheric Chemistry and Physics-from Air Pollution to Climate Change (New York: Wiley)

[141] Adamson A 1982 Physical Chemistry of Surfaces 4th edn (New York: Wiley)

[142] Carey V P 2008 Liquid-Vapor Phase-Change Phenomena (London: Taylor and Francis)

[143] Tester J W and Modell M 1997 Thermodynamics and Its Applications 3rd edn (Englewood Cliffs, NJ: Prentice Hall)

[144] Prausnitz J, Lichtenthaler R and de Azevedo E 1986 Molecular Thermodynamics of Fluid-Phase Equilibria (Englewood Cliffs, NJ: Prentice Hall)

[145] Deen W M 2012 Analysis of Transport Phenomena (Oxford: Oxford University Press)

[146] de Groot S R and Mazur P 1984 Non-Equilibrium Thermodynamics (New York: Dover)

[147] Garrett B C, Schenter G K and Morita A 2006 Molecular simulations of the transport of molecules across the liquid/ vapor interface of water Chem. Rev. 106 1355-74

[148] Gibbs J W 1877 On the equilibrium of heterogeneous substances (2) Trans. Connect. Acad. 3 343-524

[149] Gibbs J W 1876 On the equilibrium of heterogeneous substances (1) Trans. Connect. Acad. 3 108-248

[150] Lewis G 1901 The law of physico-chemical change Proc. Natl Acad. Sci. 3749

[151] Guggenheim E 1959 Thermodynamics—an Advanced Treatment for Chemists and Physicists 4th edn (Amsterdam: North-Holland)

[152] Carey V P and Wemhoff A P 2004 Relationships among liquid-vapor interfacial region properties: predictions of a thermodynamic model Int. J. Thermophys. 25 753-86

[153] Garrett B C 2004 Ions at the air/water interface Science $3031146-7$

[154] Morita A and Garrett B C 2008 Molecular theory of mass transfer kinetics and dynamics at gas-water interface Fluid Dyn. Res. 40 459-73 
[155] Jungwirth P and Tobias D J 2006 Specific ion effects at the air/water interface Chem. Rev. 106 1259-81

[156] Kolb C E et al 2010 An overview of current issues in the uptake of atmospheric trace gases by aerosols and clouds Atmos. Chem. Phys. 10 10561-605

[157] Poschl U, Rudich Y and Ammann M 2007 Kinetic model framework for aerosol and cloud surface chemistry and gas-particle interactions-part 1: general equations, parameters, and terminology Atmos. Chem. Phys. 7 5989-6023

[158] Shiraiwa M, Sosedova Y, Rouviere A, Yang H, Zhang Y, Abbatt J P D, Ammann M and Poeschl U 2011 The role of long-lived reactive oxygen intermediates in the reaction of ozone with aerosol particles Nat. Chem. 3 291-5

[159] Shiraiwa M, Pfrang C, Koop T and Poeschl U 2012 Kinetic multi-layer model of gas-particle interactions in aerosols and clouds (KM-GAP): linking condensation, evaporation and chemical reactions of organics, oxidants and water Atmos. Chem. Phys. 12 2777-94

[160] Sander R 1999 Compilation of Henry's law constants for inorganic and organic species of potential importance in environmental chemistry (version 3) www.henrys-law.org/ henry-3.0.pdf

[161] Ammann M, Cox R A, Crowley J N, Jenkin M E, Mellouki A, Rossi G E, Troe J and Wallington T J 2013 Evaluated kinetic and photochemical data for atmospheric chemistry: volume VI-heterogeneous reactions with liquid substrates Atmos. Chem. Phys. 13 8045-228

[162] Julin J, Shiraiwa M, Miles R E H, Reid J P, Poschl U and Riipinen I 2013 Mass accommodation of water: bridging the gap between molecular dynamics simulations and kinetic condensation models J. Phys. Chem. A 117 410-20

[163] Minakata D, Li K, Westerhoff P and Crittenden J 2009 Development of a group contribution method to predict aqueous phase hydroxyl radical (HO center dot) reaction rate constants Environ. Sci. Technol. 43 6220-7

[164] Cooper P L and Abbatt J P D 1996 Heterogeneous interactions of $\mathrm{OH}$ and $\mathrm{HO}_{2}$ radicals with surfaces characteristic of atmospheric particulate matter J. Phys. Chem. 100 2249-54

[165] Davis E J 2006 A history and state-of-the-art of accommodation coefficients Atmos. Res. 82 561-78

[166] Takami A, Kato S, Shimono A and Koda S 1998 Uptake coefficient of $\mathrm{OH}$ radical on aqueous surface Chem. Phys. $231215-27$

[167] Thomas J M and Thomas W J 1997 Principles and Practice of Heterogeneous Catalysis (New York: $\mathrm{VCH}$ )

[168] Chang C H and Franses E I 1992 Modified langmuirhinshelwood kinetics for dynamic adsorption of surfactants at the air-water-interface Colloids Surf. 69 189-201

[169] Chang C H and Franses E I 1995 Adsorption dynamics of surfactants at the air/water interface - a critical-review of mathematical-models, data, and mechanisms Colloids Surf. A $1001-45$

[170] Neyts E C, Yusupov M, Verlackt C C and Bogaerts A 2014 Computer simulations of plasma-biomolecule and plasmatissue interactions for a better insight in plasma medicine J. Phys. D: Appl. Phys. 47293001

[171] Codorniu-Hernandez E and Kusalik P G 2011 Insights into the solvation and mobility of the hydroxyl radical in aqueous solution J. Chem. Theory Comput. 7 3725-32

[172] Codorniu-Hernandez E and Kusalik P G 2012 Mobility mechanism of hydroxyl radicals in aqueous solution via hydrogen transfer J. Am. Chem. Soc. 134 532-8

[173] Murdachaew G, Varner M E, Phillips L F, Finlayson-Pitts B J and Gerber R B 2013 Nitrogen dioxide at the air-water interface: trapping, absorption, and solvation in the bulk and at the surface Phys. Chem. Chem. Phys. 15 204-12

[174] Kastner J 2011 Umbrella sampling WIRs Computat. Mol. Sci. 1 932-42

[175] Christ C D, Mark A E and van Gunsteren W F 2010 Feature article basic ingredients of free energy calculations: a review J. Comput. Chem. 31 1569-82

[176] Kumar S, Bouzida D, Swendsen R H, Kollman P A and Rosenberg J M 1992 The weighted histogram analysis method for free-energy calculations on biomolecules 1 . The method J. Comput. Chem. 13 1011-21

[177] Kastner J and Thiel W 2005 Bridging the gap between thermodynamic integration and umbrella sampling provides a novel analysis method: 'umbrella integration' J. Chem. Phys. 123144104

[178] Yusupov M, Neyts E C, Simon P, Berdiyorov G, Snoeckx R, van Duin A C T and Bogaerts A 2014 Reactive molecular dynamics simulations of oxygen species in a liquid water layer of interest for plasma medicine J. Phys. D: Appl. Phys. 47025205

[179] Vacha R, Slavicek P, Mucha M, Finlayson-Pitts B J and Jungwirth P 2004 Adsorption of atmospherically relevant gases at the air/water interface: free energy profiles of aqueous solvation of $\mathrm{N}_{2}, \mathrm{O}_{2}, \mathrm{O}_{3}, \mathrm{OH}, \mathrm{H}_{2} \mathrm{O}, \mathrm{HO}_{2}$, and $\mathrm{H}_{2} \mathrm{O}_{2}$ J. Phys. Chem. A 108 11573-9

[180] Wick C D and Dang L X 2007 Hydroxyl radical transfer between interface and bulk from transition path sampling Chem. Phys. Lett. 444 66-70

[181] Morita A, Kanaya Y and Francisco J S 2004 Uptake of the $\mathrm{HO}_{2}$ radical by water: Molecular dynamics calculations and their implications for atmospheric modeling J. Geophys. Res. 109 D09201

[182] Martins-Costa M T C and Ruiz-Lopez M F 2007 Molecular dynamics of hydrogen peroxide in liquid water using a combined quantum/classical force field Chem. Phys. 332 341-7

[183] Martins-Costa M T C, Anglada J M, Francisco J S and Ruiz-Lopez M F 2012 Reactivity of atmospherically relevant small radicals at the air-water interface Angew. Chem. Int. Ed. 51 5413-7

[184] Buxton G V, Greenstock C L, Helman W P and Ross A B 1988 Critical review of rate constants for reactions of hydrated electrons, hydrogen atoms and hydroxyl radicals in aqueous solutions J. Phys. Chem. Ref. Data 17 513-614

[185] Chalmet S and Ruiz-Lopez M F 2006 The structures of ozone and $\mathrm{HO}_{x}$ radicals in aqueous solution from combined quantum/classical molecular dynamics simulations J. Chem. Phys. 124194502

[186] Poling B, Prausnitz J and O'Connell J 2000 The Properties of Gases and Liquids (New York: McGraw-Hill)

[187] Hirschfelder J O, Curtiss C F and Bird R B 1954 Molecular Theory of Gases and Liquids (New York: Wiley)

[188] Chen C, Liu D X, Liu Z C, Yang A J, Chen H L, Shama G and Kong M G 2014 A model of plasma-biofilm and plasma-tissue interactions at ambient pressure Plasma Chem. Plasma Process. 34 403-41

[189] Jungwirth P and Tobias D J 2002 Ions at the air/water interface J. Phys. Chem. B 106 6361-73

[190] Dang Van Sung Mussard M, Foucher E and Rousseau A 2015 Charge and energy transferred from a plasma jet to liquid and dielectric surfaces J. Phys. D: Appl. Phys. 48424003

[191] Nikiforov A Y 2008 Plasma sputtering of water molecules from the liquid phase by low-energy ions: molecular dynamics simulation High Energy Chem. 42 235-9

[192] Minagawa Y, Shirai N, Uchida S and Tochikubo F 2014 Analysis of effect of ion irradiation to liquid surface on water molecule kinetics by classical molecular dynamics simulation Japan. J. Appl. Phys. 53010210 
[193] Babaeva N Y and Kushner M J 2011 Ion energy and angular distributions onto polymer surfaces delivered by dielectric barrier discharge filaments in air: I. Flat surfaces Plasma Source Sci. Technol. 20035017

[194] Cserfalvi T and Mezei P 1996 Operating mechanism of the electrolyte cathode atmospheric glow discharge Fresenius J. Anal. Chem. 355 813-9

[195] Hart E J and Anbar M 1970 The Hydrated Electron (New York: Wiley)

[196] Gaisin A F and Son E E 2005 Vapor-air discharges between electrolytic cathode and metal anode at atmospheric pressure High Temp. 43 1-7

[197] Paik D H, Lee I-R, Yang D-S, Baskin J S and Zewail A H 2004 Electrons in finite-sized water cavities: hydration dynamics observed in real time Science 306672

[198] Kimura Y, Alfano J C, Walhout P K and Barbara P F 1994 Ultrafast transient absorption spectroscopy of the solvated electron in water J. Phys. Chem. 983450

[199] Rumbach P, Bartels D M, Sankaran R M and Go D B 2015 The solvation of electrons by an atmospheric pressure plasma Nat. Commun. 67248

[200] Rumbach P, Bartels D M, Sankaran R M and Go D B 2015 The effect of air on solvated electron chemistry at a plasma/liquid interface J. Phys. D: Appl. Phys. 48424001

[201] Farhataziz H and Rodgers M A J 1987 Radiation Chemistry, Principles and Applications (New York: $\mathrm{VCH}$ )

[202] Siefermann K R, Liu Y X, Lugovoy E, Link O, Faubel M, Buck U, Winter B and Abel B 2010 Binding energies, lifetimes and implications of bulk and interface solvated electrons in water Nat. Chem. 2 274-9

[203] Buchner F, Schultz T and Lubcke A 2012 Solvated electrons at the water-air interface: surface versus bulk signal in low kinetic energy photoelectron spectroscopy Phys. Chem. Chem. Phys. 14 5837-42

[204] Horio T, Shen H, Adachi S and Suzuki T 2012 Photoelectron spectra of solvated electrons in bulk water, methanol, and ethanol Chem. Phys. Lett. 535 12-6

[205] Young R M and Neumark D M 2012 Dynamics of solvated electrons in clusters Chem. Rev. 112 5553-77

[206] Alizadeh E and Sanche L 2012 Precursors of solvated electrons in radiobiological physics and chemistry Chem. Rev. 112 5578-602

[207] Turi L and Rossky P J 2012 Theoretical studies of spectroscopy and dynamics of hydrated electrons Chem. Rev. 112 5641-74

[208] Uhlig F, Marsalek O and Jungwirth P 2013 Electron at the surface of water: dehydrated or not? J. Phys. Chem. Lett. $4338-43$

[209] Anbar M, Bambenek M and Ross A 1973 Selected specific rates of reaction of transients from water in aqueous solution. 1. Hydrated electron. US Department of Commerce, National Bureau of Standards (www.nist.gov/ data/nsrds/NSRDS-NBS-43.pdf)

[210] Anbar M 1968 The reactions of hydrated electrons with inorganic compounds $Q$. Rev. Chem. Soc. 22 578-98

[211] Mitra S, Svrcek V, Mariotti D, Velusamy T, Matsubara K and Kondo M 2014 Microplasma-induce liquid chemistry for stabilizing of silicon nanocrystals optical properties in water Plasma Proc. Polym. 11 158-63

[212] Hare P M, Price E A, Stanisky C M, Janik I and Bartels D M 2010 Solvated electron extinction coefficient and oscillator strength in high temperature water J. Phys. Chem. A 114 1766-75

[213] Jou F Y and Freeman G R 1979 Temperature and isotope effects on the shape of the optical-absorption spectrum of solvated electrons in water J. Phys. Chem. 83 2383-7

[214] Coe J V, Williams S M and Bowen K H 2008 Photoelectron spectra of hydrated electron clusters versus cluster size: connecting to bulk Int. Rev. Phys. Chem. 27 27-51
[215] Garrett B C et al 2005 Role of water in electron-initiated processes and radical chemistry: issues and scientific advances Chem. Rev. 105 355-89

[216] Lubcke A, Buchner F, Heine N, Hertel I V and Schultz T 2010 Time-resolved photoelectron spectroscopy of solvated electrons in aqueous NaI solution Phys. Chem. Chem. Phys. 12 14629-34

[217] Shreve A T, Yen T A and Neumark D M 2010 Photoelectron spectroscopy of hydrated electrons Chem. Phys. Lett. $493216-9$

[218] Tang Y, Shen H, Sekiguchi K, Kurahashi N, Mizuno T, Suzuki Y I and Suzuki T 2010 Direct measurement of vertical binding energy of a hydrated electron Phys. Chem. Chem. Phys. 12 3653-5

[219] Verlet J R R, Bragg A E, Kammrath A, Cheshnovsky O and Neumark D M 2005 Observation of large water-cluster anions with surface-bound excess electrons Science 307 93-6

[220] Coe J V 2001 Fundamental properties of bulk water from cluster ion data Int. Rev. Phys. Chem. 20 33-58

[221] Uhlig F, Marsalek O and Jungwirth P 2012 Unraveling the complex nature of the hydrated electron J. Phys. Chem. Lett. 3 3071-5

222] Jacobson L D and Herbert J M 2011 Theoretical characterization of four distinct isomer types in hydrated-electron clusters, and proposed assignments for photoelectron spectra of water cluster anions J. Am. Chem. Soc. 133 19889-99

[223] Marsalek O, Uhlig F, Vandevondele J and Jungwirth P 2012 Structure, dynamics, and reactivity of hydrated electrons by ab initio molecular dynamics Acc. Chem. Res. $4523-32$

[224] Sagar D M, Bain C D and Verlet J R R 2010 Hydrated electrons at the water/air interface J. Am. Chem. Soc. 132 6917-9

[225] Faubel M, Steiner B and Toennies J P 1997 Photoelectron spectroscopy of liquid water, some alcohols, and pure nonane in free micro jets J. Chem. Phys. 1069013

[226] Marsalek O, Uhlig F, Frigato T, Schmidt B and Jungwirth P 2010 Dynamics of electron localization in warm versus cold water clusters Phys. Rev. Lett. 105043002

[227] Caprasecca S, Gorfinkiel J D, Bouchiha D and Caron L G 2009 Multiple scattering approach to elastic electron collisions with molecular clusters J. Phys. B: At. Mol. Opt. Phys. 42095205

[228] Bouchiha D, Caron L G, Gorfinkiel J D and Sanche L 2008 Multiple scattering approach to elastic low-energy electron collisions with the water dimer J. Phys. B: At. Mol. Opt. Phys. 41045204

[229] Dau H, Limberg C, Reier T, Risch M, Roggan S and Strasser P 2010 The mechanism of water oxidation: from electrolysis via homogeneous to biological catalysis ChemCatChem 2 724-61

[230] Hickling A 1949 The mechanism of electrode processes in aqueous solutions $Q$. Rev. 3 95-125

[231] Denaro A R and Hickling A 1957 Glow-discharge electrolysis in aqueous solutions J. Electrochem. Soc. $104 \mathrm{C} 170$

[232] Hickling A 1971 Modern Aspects of Electrochemistry ed J O M Bockris and B E Conway (New York: Plenum) pp 329-73

[233] Kirkpatrick M and Locke B 2005 Hydrogen, oxygen, and hydrogen peroxide formation in aqueous phase pulsed corona electrical discharge Ind. Eng. Chem. Res. 44 4243-8

[234] Richmonds C, Witzke M, Bartling B, Lee S W, Wainright J, Liu C C and Sankaran R M 2011 Electron transfer reactions at the plasma-liquid interface J. Am. Chem. Soc. 133 17582-5 
[235] Elahi A and Caruana D J 2013 Plasma electrochemistry: voltammetry in a flame plasma electrolyte Phys. Chem. Chem. Phys. 15 1108-14

[236] Fowowe T, Hadzifejzovic E, Hu J P, Foord J S and Caruana D J 2012 Plasma electrochemistry: development of a reference electrode material for high temperature plasma Adv. Mater. 24 6305-9

[237] Witzke M, Rumbach P, Go D B and Sankaran R M 2012 Evidence for the electrolysis of water by atmosphericpressure plasmas formed at the surface of aqueous solutions J. Phys. D: Appl. Phys. 45442001

[238] Sengupta S and Prakash Singh O 1994 Contact glow discharge electrolysis: a study of its chemical yields in aqueous inert-type electrolytes J. Electroanal. Chem. 369 113-20

[239] Hofman-Caris R C H M, Harmsen D J H, Beerendonk E F, Knol T H, Houtman C J, Metz D H and Wols B A 2012 Prediction of advanced oxidation performance in pilot UV/ $\mathrm{H}_{2} \mathrm{O}_{2}$ reactor systems with MP- and LP-UV lamps Chem. Eng. J. 210 520-8

[240] Bagheri M and Mohseni M 2014 Computational fluid dynamics (CFD) modeling of VUV/UV photoreactors for water treatment Chem. Eng. J. 256 51-60

[241] Gonzalez M A, Oliveros E, Wörner M and Braun A M 2004 Vacuum-ultraviolet photolysis of aqueous reaction systems J. Photochem. Photobiol. C 5 225-46

[242] Wols B A and Hofmann-Caris C H M 2012 Review of photochemical reaction constants of organic micropollutants required for $\mathrm{UV}$ advanced oxidation processes in water Water Res. 46 2815-27

[243] Coenen T, Van de Moortel W, Logist F, Luyten J, Van Impe J F M and Degreve J 2013 Modeling and geometry optimization of photochemical reactors: single-and multilamp reactors for $\mathrm{UV}-\mathrm{H}_{2} \mathrm{O}_{2}$ AOP systems Chem. Eng. Sci. 96 174-89

[244] Rosenthal I, Sostaric J Z and Riesz P 2004 Enlightened sonochemistry Res. Chem. Intermed. 30 685-701

[245] Willberg D M, Lang P S, Höchemer R H, Kratel A and Hoffmann M R 1996 Degradation of 4-chlorophenol, 3,4-dichloroaniline, and 2,4,6-trinitrotoluene in an electrohydraulic discharge reactor Environ. Sci. Technol. 30 2526-34

[246] Lang P S, Ching W K, Willberg D M and Hoffmann M R 1998 Oxidative degradation of 2,4,6-trinitrotoluene by ozone in an electrohydraulic discharge reactor Environ. Sci. Technol. 32 3142-8

[247] Ching W K, Colussi A J, Sun H J, Nealson K H and Hoffmann M R 2001 Escherichia coli disinfection by electrohydraulic discharges Environ. Sci. Technol. 35 4139-44

[248] Martin E A 1960 Experimental investigation of a high-energy density, high-pressure arc plasma J. Appl. Phys. 31 255-67

[249] Robinson J W, Ham M and Balaster A N 1973 Ultraviolet radiation from electrical discharges in water J. Appl. Phys. $4472-5$

[250] Lukes P, Clupek M, Babicky V and Sunka P 2008 Ultraviolet radiation from the pulsed corona discharge in water Plasma Source Sci. Technol. 17024012

[251] Lu X and Laroussi M 2005 Optimization of ultraviolet emission and chemical species generation from a pulsed dielectric barrier discharge at atmospheric pressure J. Appl. Phys. 98023301

[252] Laroussi M and Leipold F 2004 Evaluation of the roles of reactive species, heat, and UV radiation in the inactivation of bacterial cells by air plasmas at atmospheric pressure Int. J. Mass Spectrom. 233 81-6

[253] Shuaibov A K, Shimon L L, Dashchenko A I and Shevera I V 2001 A water-vapor electric-discharge vacuum ultraviolet source Tech. Phys. Lett. 27 642-3
[254] Shuaibov A K, Minya A J, Gomoki Z T, Shevera I V and Gritsak R V 2011 Vacuum-UV emitter using low-pressure discharge in helium-water vapor mixture Tech. Phys. Lett. 37 126-7

[255] Foest R, Bindemann T, Brandenburg R, Kindel E, Lange H, Stieber M and Weltmann K D 2007 On the vacuum ultraviolet radiation of miniaturized non-thermal atmospheric pressure plasma jet Plasma Proc. Polym. 4 S460-S4

[256] Reuter S, Niemi K, Schulz-Von Der Gathen V and Döbele H F 2009 Generation of atomic oxygen in the effluent of an atmospheric pressure plasma jet Plasma Source Sci. Technol. 18015006

[257] Brandenburg R, Lange H, von Woedtke T, Stieber M, Kindel E, Ehlbeck J and Weltmann K D 2009 Antimicrobial effects of UV and VUV radiation of nonthermal plasma jets IEEE Trans. Plasma Sci. 37 877-83

[258] Cantrell C A, Zimmer A and Tyndall G S 1997 Absorption cross sections for water vapor from 183 to $193 \mathrm{~nm}$ Geophys. Res. Lett. $242195-8$

[259] Crapulli F, Santoro D, Sasges M R and Ray A K 2014 Mechanistic modeling of vacuum UV advanced oxidation process in an annular photoreactor Water Res. 64 209-25

[260] Watanabe K and Zelikoff M 1953 Absorption coefficients of water vapor in the vacuum ultraviolet J. Opt. Soc. Am. $43753-5$

[261] Oppenländer T, Walddörfer C, Burgbacher J, Kiermeier M Lachner K and Weinschrott H 2005 Improved vacuum-UV (VUV)-initiated photomineralization of organic compounds in water with a xenon excimer flow-through photoreactor (Xe2* lamp, $172 \mathrm{~nm}$ ) containing an axially centered ceramic oxygenator Chemosphere 60 302-9

[262] Gettoff N 1993 Purification of drinking water by irradiation. A review Proc. Ind. Acad. Sci. Chem/Sci. 105373

[263] Heit G, Neuner A, Saugy P Y and Braun A M 1998 Vacuum-UV (172 nm) actinometry. The quantum yield of the photolysis of water J. Phys. Chem. A 102 5551-61

[264] Oppenländer T 2003 Photochemical Purification of Water and Air (New York: Wiley)

[265] Seinfeld J H and Pandis S N 1998 Atmospheric Chemistry and Physics (New York: Wiley)

[266] Stief L J, Payne W A and Klemm R B 1975 A flash photolysis-resonance fluorescence study of the formation of $\mathrm{O}\left({ }^{1} \mathrm{D}\right)$ in the photolysis of water and the reaction of $\mathrm{O}\left({ }^{1} \mathrm{D}\right)$ with $\mathrm{H}_{2}$, Ar, and $\mathrm{He} J$. Chem. Phys. 62 4000-8

[267] Wu C Y R and Chen F Z 1993 Velocity distributions of hydrogen atoms and hydroxyl radicals produced through solar photodissociation of water J. Geophys. Res. 987415

[268] Yuan K, Cheng L, Cheng Y, Guo Q, Dai D and Yang X 2009 Two-photon photodissociation dynamics of $\mathrm{H}_{2} \mathrm{O}$ via the D electronic state J. Chem. Phys. 131074301

[269] Bugaenko V L and Byakov V M 1998 Quantitative model of the radiolysis of liquid water and dilute solutions of hydrogen, oxygen, and hydrogen peroxide. I. Formulation of the model High Energy Chem. 33407

[270] Jablonowski H, Bussiahn R, Hammer M U, Weltmann K D, von Woedtke T and Reuter S 2014 Impact of plasma jet VUV-radiation on reactive oxygen species generation in bio-relevant liquids Phys. Plasmas 22122008

[271] Piskarev I M 2000 Model reactions accompanying a corona discharge in the $\mathrm{O}_{2}(\mathrm{~g})-\mathrm{H}_{2} \mathrm{O}$ system Russ. J. Phys. Chem. 74 546-51

[272] Zvereva G N 2012 Using vacuum ultraviolet radiation to obtain highly reactive radicals $J$. Opt. Technol. 79 477-83

[273] Sério S, Nunes Y, Hoffmann S V, Mason N J, Duflot D and Limao-Vieira P 2012 Electronic state spectroscopy of 1,4-pentadiene as studied by VUV photoabsorption 
spectroscopy and ab initio calculations J. Phys. Chem. A $1168176-84$

[274] Legrini O, Oliveros E and Braun A M 1993 Photochemical processes for water treatment Chem. Rev. 93 671-98

[275] Bolton J R and Cater S R 1994 Aquatic and Surface Photochemistry ed G R Heiz et al (Boca Raton, FL: Lewis Publishers) pp 467-90

[276] Tarr M A 2003 Chemical Degradation Methods for Wastes and Pollutants: Environmental and Industrial Applications (New York: Dekker)

[277] Peyton G R and Glaze W H 1988 Destruction of pollutants in water with ozone in combination with ultraviolet radiation. 3. Photolysis of aqueous ozone Environ. Sci. Technol. 22 761-7

[278] Cho M, Cates E L and Kim J-H 2011 Inactivation and surface interactions of MS-2 bacteriophage in $\mathrm{a} \mathrm{TiO}_{2}$ photoelectrocatalytic reactor Water Res. 45 2104-10

[279] Hussain M, Ceccarelli R, Marchisio D L, Fino D, Russo N and Geobaldo F 2010 Synthesis, characterization, and photocatalytic application of novel $\mathrm{TiO}_{2}$ nanoparticles Chem. Eng. J. 157 45-51

[280] Chong M N, Jin B, Chow C W K and Saint C 2010 Recent developments in photocatalytic water treatment technology: a review Water Res. 44 2997-3027

[281] Watts M J and Linden K G 2008 Photooxidation and subsequent biodegradability of recalcitrant tri-alkyl phosphates TCEP and TBP in water Water Res. 42 4949-54

[282] Lukes P, Clupek M, Sunka P, Peterka F, Sano T, Negishi N, Matsuzawa S and Takeuchi K 2005 Degradation of phenol by underwater pulsed corona discharge in combination with $\mathrm{TiO}_{2}$ photocatalysis Res. Chem. Intermed. 31 285-94

[283] Hao X L, Zhou M H and Lei L C 2007 Non-thermal plasmainduced photocatalytic degradation of 4-chlorophenol in water J. Hazard. Mater. 141 475-82

[284] Wang H, Li J, Quan X, Wu Y, Li G and Wang F 2007 Formation of hydrogen peroxide and degradation of phenol in synergistic system of pulsed corona discharge combined with $\mathrm{TiO}_{2}$ photocatalysis J. Hazard. Mater. $141336-43$

[285] Zeleny J 1917 Instability of electrified surfaces Phys. Rev. $101-6$

[286] Melcher J R and Smith C V 1969 Electrohydrodynamic charge relaxation and interfacial perpendicular field instability Phys. Fluids $\mathbf{1 2} 778$

[287] Jones T B and Bliss G W 1977 Bubble dielectrophoresis J. Appl. Phys. 481412

[288] Watson P K 1985 Electrostatic and hydrodynamic effects in electrical breakdown of liquid dielectrics IEEE Trans. Ind. Appl. El-20 396

[289] Hase M, Watannabe S N and Yoshikawa K 2006 Rhythmic motion of a droplet under a DC electric field Phys. Rev. E 74046301

[290] Korobeinikov S, Melekhov A V, Sinikh Y N and Soloveichik Y G 2001 Effect of strong electric fields on the behavior of bubbles in water High Temp. 39368

[291] Bruggeman P J, Graham L M, Degroote J, Vierendeels J and Leys C 2007 Water surface deformation in strong electrical fields and its influence on electrical breakdown in a metal pin-water electrode system J. Phys. D: Appl. Phys. 40 4779-86

[292] Sugimoto T and Higashiyama Y 2001 Production of water drops and corona due to rupture of air bubbles at water surface under a positive dc electric field J. Electrost. 2009219

[293] Oehmigen K, Hoder T, Wilke C, Brandenburg R, Hahnel M, Weltmann K D and Woedke T 2011 Volume effects of atmospheric pressure plasma in liquids IEEE Trans. Plasma Sci. 392646
[294] Thompson L H and Doraiswamy L K 1999 Sonochemistry: science and engineering Ind. Eng. Chem. Res. 381215

[295] Robinson J A, Bergougnou M A, Castle G S P and Inculet I I 2002 A nonlinear model of AC-field-induced parametric waves on a water surface IEEE Trans. Ind. Appl. 38 379-88

[296] Shirai N, Sekine R, Uchida S and Tochikubo F 2014 Atmospheric negative corona discharge using Taylor cone as a liquid cathode Japan. J. Appl. Phys. 53026001

[297] Taylor G 1969 Electrically driven jets Proc. R. Soc. A $313453-75$

[298] Eggers J and Villermaux E 2008 Physics of liquid jets Rep. Prog. Phys. 71036601

[299] Schwartz A J, Ray S J, Elish E, Storey A P, Rubinshtein A A, Chan G C Y, Pfeuffer K P and Hieftje G M 2012 Visual observations of an atmospheric-pressure solution-cathode glow discharge Talanta 102 26-33

[300] Nguyen A V and Evans G M 2006 Computational fluid dynamics modeling of gas jets impinging onto liquid pools Appl. Math. Modelling 30 1472-84

[301] van Rens J F M, Schoof J T, Ummelen F C, van Vught D C, Bruggeman P J and van Veldhuizen E M 2014 Induced liquid phase flow by RF Ar cold atmospheric pressure plasma jet IEEE Trans. Plasma Sci. 422622

[302] Iseni S, Schmidt-Bleker A, Winter J, Weltmann K D and Reuter S 2014 Atmospheric pressure turbulent argon air boundary in a $\mathrm{MHz}$ argon plasma jet investigated by OH-tracer PLIF spectroscopy J. Phys. D: Appl. Phys. 47152001

[303] Sommers B, Foster J E and Kushner M J 2011 Observations of electric discharge streamer propagation and capillary oscillations on the surface of air bubbles in water J. Phyics D: Appl. Phys. 44082001

[304] Sommers B S 2013 Plasma ignition in underwater gas bubbles $P h D$ Thesis University of Michigian

[305] Sommers B and Foster J E 2012 Nonlinear oscillation of gas bubbles submerged in water: implications for plasma breakdown J. Phys. D: Appl. Phys. 45415203

[306] Foster J E, Weatherford B, Gillman E and Yee B 2010 Underwater operation of a DBD plasma jet Plasma Source Sci. Technol. 19025001

[307] Schaper L, Graham W G and Stalder K R 2011 Vapour layer formation by electrical discharges through electrically conducting liquids-modelling and experiment Plasma Source Sci. Technol. 20034003

[308] Jiang N, Yang J, He F and Cao Z 2014 Interplay of discharge and gas flow in atmospheric pressure plasma jets J. Appl. Phys. 47152001

[309] Robert E, Sarron V, Darny T, Ries D, Dozias S, Fontaine J, Joly L and Pouvesle J-M 2014 Rare gas flow structuration in plasma jet experiments Plasma Source Sci. Technol. 23012003

[310] Foletto M, Puech V, Fontaine J, Joly L and Pitchford L C 2014 Evidence of the influence of plasma jets on a helium flow into open air IEEE Trans. Plasma Sci. 422436

[311] Shimizu T, Iwafuchi Y, Morfill G E and Sato T 2011 Formation of thermal flow fields and chemical transport in air and water by atmospheric plasma New J. Phys. 13053025

[312] Manz A, Effenhauser C S, Burggraf N, Harisson D J, Seiler K and Fluri K 1994 Electroosmotic pumping and electrophoretic separations for miniaturized chemical analysis systems J. Micromech. Microeng. 4 257-65

[313] Ramos A 2011 Electrokinetics and Electrohydrodynamics in Microsystems (Berlin: Springer)

[314] Levich V G and Krylov V S 1969 Surface-tension driven phenomena Ann. Rev. Fluid Mech. $1293-316$

[315] Cussler E L 1984 Diffusion: Mass Transfer in Fluid Systems (Cambridge: Cambridge University Press) 
[316] Abbatt J P D, Leea A K Y and Thornton J A 2012 Quantifying trace gas uptake to tropospheric aerosol: recent advances and remaining challenges Chem. Soc. Rev. 41 6555-81

[317] Berkemeier T, Huisman A J, Ammann M, Shiraiwa M, Koop T and Poeschl U 2013 Kinetic regimes and limiting cases of gas uptake and heterogeneous reactions in atmospheric aerosols and clouds: a general classification scheme Atmos. Chem. Phys. 13 6663-86

[318] Shiraiwa M, Berkemeier T, Shilling-Fahnestock K A, Seinfeld J H and Poeschl U 2014 Molecular corridors and kinetic regimes in the multiphase chemical evolution of secondary organic aerosol Atmos. Chem. Phys. 14 8323-41

[319] Valsaraj K T 2012 A review of the aqueous aerosol surface chemistry in the atmospheric context Open J. Phys. Chem. $258-66$

[320] Frank T C, Arturo S G and Golden B S 2014 Framework for correlating the effect of temperature on nonelectrolyte and ionic liquid activity coefficients AIChE J. 60 3675-90

[321] Astarita G, Savage D W and Bisio A 1983 Gas Treating with Chemical Solvents (New York: Wiley)

[322] Danckwerts P V 1970 Gas-Liquid Reactions (New York: McGraw-Hill)

[323] Lindsay A, Anderson C, Slikboer E, Shannon S and Graves D 2015 Momentum, heat and neutral mass transport in convective atmospheric pressure plasma-liquid systems and implications for aqueous targets J. Phys. D: Appl. Phys. 48424007

[324] Verreycken T, van Gessel A F H, Pageau A and Bruggeman P 2011 Validation of gas temperature measurements by OES in an atmospheric air glow discharge with water electrode using Rayleigh scattering Plasma Source Sci. Technol. 20024002

[325] Dorai R and Kushner M J 2003 A model for plasma modification of polypropylene using atmospheric pressure discharges J. Phys. D: Appl. Phys. 36 666-85

[326] Stalder K R, Vidmar R J, Nersisyan G and Graham W G 2006 Modeling the chemical kinetics of high-pressure glow discharges in mixtures of helium with real air J. Appl. Phys. 99093301

[327] Liu D X, Bruggeman P J, Iza F, Rong M Z and Kong M G 2010 Global model of low-temperature atmosphericpressure $\mathrm{He}+\mathrm{H}_{2} \mathrm{O}$ plasmas Plasma Source Sci. Technol. 19025018

[328] Liu D X, Yang A J, Wang X H, Rong M Z, Iza F and Kong M G 2012 Wall fluxes of reactive oxygen species of an rf atmospheric-pressure plasma and their dependence on sheath dynamics J. Phys. D: Appl. Phys. 45305205

[329] van Veldhuizen E M 1999 Electrical Discharges for Environmental Purposes: Fundamentals and Applications (New York: Nova Science Publishers)

[330] Murakami T, Niemi K, Gans T, O'Connell D and Graham W G 2013 Interacting kinetics of neutral and ionic species in an atmospheric-pressure helium-oxygen plasma with humid air impurities Plasma Source Sci. Technol. 22045010

[331] van Gaens W and Bogaerts A 2013 Kinetic modelling for an atmospheric pressure argon plasma jet in humid air J. Phys. D: Appl. Phys. 46275201

[332] Xiong Z, Robert E, Sarron V, Pouvesle J-M and Kushner M J 2012 Dynamics of ionization wave splitting and merging of atmospheric-pressure plasmas in branched dielectric tubes and channels J. Phys. D: Appl. Phys. 45275201

[333] Soloshenko I A, Tsiolko V V, Pogulay S S, Kalyuzhnaya A G, Bazhenov V Y and Shchedrin A I 2009 Effect of water adding on kinetics of barrier discharge in air Plasma Source Sci. Technol. 18045019
[334] Zhang S, van Gaens W, van Gessel B, Hofmann S, van Veldhuizen E M, Bogaerts A and Bruggeman P J 2013 Spatially resolved ozone densities and gas temperatures in a time modulated RF driven atmospheric pressure plasma jet: an analysis of the production and destruction mechanisms J. Phys. D: Appl. Phys. 46205202

[335] Nagaraja S, Yang V and Adamovich I 2013 Multi-scale modelling of pulsed nanosecond dielectric barrier plasma discharges in plane-to-plane geometry J. Phys. D: Appl. Phys. 46155205

[336] Vasko C A, Liu D X, van Veldhuizen E M, Iza F and Bruggeman P J 2014 Hydrogen peroxide production in an atmospheric pressure RF glow discharge: comparison of models and experiments Plasma Chem. Plasma Process. 34 1081-99

[337] van Gaens W, Bruggeman P J and Bogaerts A 2014 Numerical analysis of the $\mathrm{NO}$ and $\mathrm{O}$ generation mechanism in a needle-type plasma jet New J. Phys. 16063054

[338] Kieffer L J 1973 A compilation of electron collision cross section data for modeling gas discharge lasers JILA Inf. Center Rep. 13 1-152

[339] Itikawa Y and Mason N 2005 Cross sections for electron collisions with water molecules J. Phys. Chem. Ref. Data 34 1-22

[340] Karwasz G P, Brusa R S and Zecca A 2001 One century of experiments on electron-atom and molecule scattering. A critical review of integral cross sections. II. Polyatomic molecules Riv. Nuovo Cimento 24 1-118

[341] Mark T D, Hatano Y and Linder F 1995 Electron collision cross sections in Atomic and molecular data for radiotherapy and radiation research Final Report of $a$ Co-Ordinated Research Programme IAEA-TECDOC-799 IAEA, Vienna

[342] Hayashi M 2003 Bibliography of electron and photon cross sections with atoms and molecules published in the 20th century - water vapour Nat. Inst. Fusion Res. (Report NIFS-Data-81)

[343] Pack J L, Voshall R E and Phelps A V 1962 Drift velocities of slow electrons in krypton, xenon, deuterium, carbon monoxide, carbon dioxide, water vapor, nitrous oxide and ammonia Phys. Rev. 127 2084-9

[344] Crompton R W, Rees J A and Jory R L 1965 The diffusion and attachment of electrons in water vapour Aust. J. Phys. $18541-51$

[345] Gallagher J W, Beaty E C, Dutton J and Pitchford L C 1983 An annotated compilation and appraisal of electron swarm data in electronegative gases J. Phys. Chem. Ref. Data 12 109-52

[346] Hasegawa H, Date H and Shimozuma M 2007 Electron swarm parameters in water vapour J. Phys. D: Appl. Phys. $402495-8$

[347] De Urquijo J, Basurto E, Juarez A M, Ness K F, Robson R E, Brunger M J and White R D 2014 Electron drift velocities in He and water mixtures: measurements and an assessment of the water vapour cross-section sets J. Chem. Phys. 141014308

[348] Phelps A V 2004 http://jila.colorado.edu/ avp/collision_data/ electronneutral/ELECTRON.TXT

[349] 2014 LXcat database www.lxcat.net

[350] Yousfi M and Benabdessadok M D 1996 Boltzmann equation analysis of electron-molecule collision cross sections in water vapor and ammonia J. Appl. Phys. 80 6619-30

[351] Ness K F and Robson R E 1988 Transport properties of electrons in water vapor Phys. Rev. A 38 1446-56

[352] Biagi S 2014 http://rjd.web.cern.ch/rjd/cgi-bin/cross

[353] Ness K F, Robson R E, Brunger M J and White R D 2012 Transport coefficients and cross sections for electrons in water vapour: comparison of cross section sets using an 
improved Boltzmann equation solution J. Chem. Phys. 136024318

[354] Robson R E, White R D and Ness K F 2011 Transport coefficients for electrons in water vapor: definition, measurement, and calculation J. Chem. Phys. 134064319

[355] Cheung B and Elford M T 1990 The drift velocity of electrons in water vapour at low values of E/N Aust. J. Phys. 43 755-63

[356] Sivos J, Skoro N, Maric D, Malovic G and Petrovic Z L 2015 Breakdown and dc discharge in low-pressure water vapor J. Phys. D: Appl. Phys. $\mathbf{4 8} 424011$

[357] Petrović Z Lj, Dujko S, Marić D, Malović G, Nikitović Z, Šašić O, Jovanović J, Stojanović V and RadmilovićRađenović M 2009 Measurement and interpretation of swarm parameters and their applications in plasma modelling J. Phys. D: Appl. Phys. 42194002

[358] Champion C, Hanssen J and Hervieux P A 2002 Electron impact ionization of water molecule J. Chem. Phys. 117 197-204

[359] Straub H C, Lindsay B G, Smith K A and Stebbings R F 1998 Absolute partial cross sections for electron-impact ionization of $\mathrm{H}_{2} \mathrm{O}$ and $\mathrm{D}_{2} \mathrm{O}$ from threshold to $1000 \mathrm{eV} \mathrm{J}$. Chem. Phys. 108 109-16

[360] Lindsay B G and Mangan M A 2003 Photon and Electron Interactions with Atoms, Molecules and Ions-Interactions of Photons and Electrons with Molecules ed Y Itikawa (Berlin: Springer)

[361] Milloy H B, Reid I D and Crompton R W 1975 Zero-field mobility for electrons in dry and humid air Aust. J. Phys. 28 231-4

[362] Petrovic Z L 1986 The determination of the diffusion coefficient for thermal electrons in water vapour by the use of a modified Blanc's law procedure Aust. J. Phys. 39 249-52

[363] Petrovic Z L, Raspopovic Z M, Stojanovic V D, Jovanovic J V, Malovic G, Makabe T and De Urquijo J 2007 Data and modeling of negative ion transport in gases of interest for production of integrated circuits and nanotechnologies Appl. Surf. Sci. 253 6619-40

[364] Phelps A V 1992 Collisions of $\mathrm{H}^{+}, \mathrm{H}_{2}^{+}, \mathrm{H}_{3}^{+}, \mathrm{ArH}^{+}, \mathrm{H}^{-}$, $\mathrm{H}$, and $\mathrm{H}_{2}$ with $\mathrm{Ar}$ and of $\mathrm{Ar}^{+}$and $\mathrm{ArH}^{+}$with $\mathrm{H}_{2}$ for energies from $0.1 \mathrm{eV}$ to $10 \mathrm{keV}$ J. Phys. Chem. Ref. Data 21 883-97

[365] Nakai Y, Shirai T, Tabata T and Ito R 1987 Cross sections for charge transfer of hydrogen atoms and ions colliding with gaseous atoms and molecules At. Data Nucl. Data Tables 37 69-101

[366] Ellis H W, McDaniel E W, Albritton D L, Viehland L A, Lin S L and Mason E A 1978 Transport properties of gaseous ions over a wide energy rang At. Data Nucl. Data Tables 22 179-217

[367] Skoro N, Maric D, Malovic G, Graham W G and Petrovic Z L 2011 Electrical breakdown in water vapor Phys. Rev. E 84055401

[368] Miller J H and Green A E S 1973 Proton energy degradation in water vapor Radiat. Res. 54 343-63

[369] Lozier W W 1930 Negative ions in hydrogen and water vapor Phys. Rev. 36 1417-8

[370] Stojanovic V D, Raspopovic Z M, Maric D and Petrovic Z L 2015 Cross sections and transport of $\mathrm{O}^{-}$in $\mathrm{H}_{2} \mathrm{O}$ vapor at low pressures Eur. Phys. J. D 6963

[371] De Urquijo J, Bekstein A, Ruiz-Vargas G and Gordillo-Vazquez F J 2013 Drift and clustering of daughter negative ions of $\mathrm{H}_{2} \mathrm{O}$ in parent gas J. Phys. D: Appl. Phys. 46035201

[372] Harrison R G and Aplin K L 2007 Water vapour changes and atmospheric cluster ions Atmos. Res. 85 199-208

[373] Hutton P J 1963 Growth of small ions in supersaturated water vapour Proc. Phys. Soc. 82 222-30
[374] Viggiano A A, Morris R A and Mason E A 1993 Mobilities and interaction potentials for the $\mathrm{O}^{+}-\mathrm{He}$ and $\mathrm{O}^{-}-\mathrm{He}$ systems J. Chem. Phys. 98 6483-7

[375] Viehland L A and Mason E A 1995 Transport properties of gaseous ions over a wide energy range At. Data Nucl. Data Tables 60 37-95

[376] Spanel P and Smith D 2000 Influence of water vapour on selected ion flow tube mass spectrometric analyses of trace gases in humid air and breath Rapid Commun. Mass Spectrom. 14 1898-906

[377] Ellis H W, Pai R Y, McDaniel E W, Mason E A and Viehland L A 1976 Transport properties of gaseous ions over a wide energy range At. Data Nucl. Data Tables 17 177-210

[378] Viehland L A and Kirkpatrick C C 1995 Relating ion/neutral reaction rate coefficients and cross-sections by accessing a database for ion transport properties Int. J. Mass Spec. Ion Proc. 149/150 555-71

[379] Moruzzi J L and Phelps A 1966 Survey of negative-ionmolecule reactions in $\mathrm{O}_{2}, \mathrm{CO}_{2}, \mathrm{H}_{2} \mathrm{O}, \mathrm{CO}$, and mixtures of these gases at high pressures J. Chem. Phys. 45 4617-27

[380] Fehsenfeld F C and Ferguson E E 1974 Laboratory studies of negative ion reaction with atmospheric trace constituents J. Chem. Phys. 61 3181-93

[381] Melton C E 1972 Formation of, by termolecular reactions, and bond dissociation energy, structure, and bond length for $\mathrm{OH}-\mathrm{H}_{2} \mathrm{O}$ and $\mathrm{O}-\mathrm{H}_{2} \mathrm{O}$ J. Phys. Chem. 76 3116-20

[382] Van Doren J M, Barlow S E, DePuy C H and Bierbaum V M 1991 Exchange reactions of $18 \mathrm{O}^{-}$with some di- and triatomic molecules Int. J. Mass Spec. Ion Proc. $109305-25$

[383] Hickel B, Corfitzen H and Sehested K 1996 Measurement of the rate constants of the reactions $\mathrm{OH}+\mathrm{OH}^{-} \mathrm{O}^{-}+\mathrm{H}_{2} \mathrm{O}$ and $\mathrm{OD}+\mathrm{OD}^{-} \mathrm{O}^{-}+\mathrm{D}_{2} \mathrm{O}$ in forward and reverse directions. Kinetic determination of the $\mathrm{pK}$ 's of $\mathrm{OH}$ and OD radicals J. Phys. Chem. 100 17186-90

[384] Stockdale J A D, Compton R N and Reinhardt P W 1968 Measurement of the cross section for the reactions $\mathrm{H}^{-}+\mathrm{H}_{2} \mathrm{O} \rightarrow \mathrm{OH}^{-}+\mathrm{H}_{2}$ and $\mathrm{D}^{-}+\mathrm{D}_{2} \mathrm{O} \rightarrow \mathrm{OD}^{-}+\mathrm{D}_{2}$ at incident ion energies near $2 \mathrm{eV}$ Phys. Rev. Lett. 21 664-7

[385] Stockdale J A D, Compton R N and Reinhardt P W 1969 Studies of negative-ion-molecule reactions in the energy region from 0 to $3 \mathrm{eV}$ Phys. Rev. 184 81-93

[386] Sieck L W, Heron J T and Green D S 2000 Chemical kinetics database and predictive schemes for humid air plasma chemistry. Part I: Positive ion-molecule reactions Plasma Chem. Plasma Process. 20 235-58

[387] Hasted J B and Smith R B 1956 The detachment of electrons from negative ions Proc. Roy. Soc. A 235 349-53

[388] Champion R L, Doverspike L D and Lam S K 1976 Electron detachment from negative ions: the effects of isotopic substitution Phys. Chem. 13 617-21

[389] Turner B R and Rutherford J A 1968 Charge transfer and ion-atom interchange reactions of water vapor ions J. Geophys. Res. 73 6751-8

[390] Skalny J D, Mikoviny T, Matejcik S and Mason N J 2004 An analysis of mass spectrometric study of negative ions extracted from negative corona discharge in air Int. $J$. Mass Spec. 233 317-24

[391] Avtaeva V, General A A and Kel'man V A 2010 Kinetic model for low-density non-stationary gas discharge in water vapour J. Phys. D: Appl. Phys. 43315201

[392] Atkinson R, Baulch D L, Cox R A, Crowley J N, Hampson R F, Hynes R G, Jenkin M E, Rossi M J and Troe J 2004 Evaluated kinetic and photochemical data for atmospheric chemistry: volume I-gas phase reactions of $\mathrm{O}_{x}, \mathrm{HO}_{x}, \mathrm{NO}_{x}$ and $\mathrm{SO}_{x}$ species Atmos. Chem. Phys. 4 1461-738 
[393] Atkinson R, Baulch D L, Cox R A, Hampson R F, Kerr J A, Rossi M J and Troe J 1997 Evaluated kinetic and photochemical data for atmospheric chemistry: supplement VI-IUPAC subcommittee on gas kinetic data evaluation for atmospheric chemistry J. Phys. Chem. Ref. Data 26 1329-499

[394] Herron J T and Green D S 2001 Chemical kinetics database and predictive schemes for nonthermal humid air plasma chemistry. Part II. Neutral species reactions Plasma Chem. Plasma Process. 21 459-81

[395] Kossyi I A, Kostinsky A Y, Matveyev A A and Silakov V P 1992 Kinetic scheme of the non-equilibrium discharge in nitrogen-oxygen mixtures Plasma Source Sci. Technol. $1207-20$

[396] Benson S W 1981 The kinetics and thermochemistry of chemical oxidation with application to combustion and flames Prog. Energy Combust. Sci. 7 125-34

[397] Zhou D D Y, Skodje R T, Liu W J and Davis M J 2013 Multi-target global sensitivity analysis of $n$-butanol combustion 8th US National Combustion Meeting (University of Utah) Paper 070RK-0090

[398] Lehmann R 2004 An algorithm for the determination of all significant pathways in chemical reaction systems J. Atmos. Chem. 47 45-78

[399] Markosyan A H, Luque A, Gordillo-Vazquez F J and Ebert U 2014 PumpKin: a tool to find principal pathways in plasma chemical models Comput. Phys. Comm. 185 2697-702

[400] Smith G P et al 2014 GRI-mech 3.0 www.me.berkeley.edu/ gri_mech/

[401] Westbrook C K, Mizobuchi Y, Poinsot T J, Smith P J and Warnatz E 2005 Computational combustion Proc. Combust. Inst. 30 125-57

[402] Dilecce G and De Benedictis S 2011 Laser diagnostics of high-pressure discharges: laser induced fluorescence detection of $\mathrm{OH}$ in $\mathrm{He} / \mathrm{Ar}-\mathrm{H}_{2} \mathrm{O}$ dielectric barrier discharges Plasma Phys. Control. Fusion 53124006

[403] Mehr F J and Biondi M A 1969 Electron temperature dependence of recombination of $\mathrm{O}_{2}^{+}$and $\mathrm{N}_{2}^{+}$ions with electrons Phys. Rev. 181 264-71

[404] Mul P M, McGowan J W, Defrance P and Mitchell J B A 1983 Merged electron-ion beam experiments: V. Dissociative recombination of $\mathrm{OH}^{+}, \mathrm{H}_{2} \mathrm{O}^{+}, \mathrm{H}_{3} \mathrm{O}^{+}$and $\mathrm{D}_{3} \mathrm{O}^{+}$J. Phys. B: At. Mol. Phys. 16 3099-107

[405] Schofield K 1978 Rate constants for the gaseous interactions of $\mathrm{O}\left(2^{1} \mathrm{D}_{2}\right)$ and $\mathrm{O}\left(2^{1} \mathrm{~S}_{0}\right)$ - a critical evaluation J. Photochem. 9 55-68

[406] Schofield K 1979 Critically evaluated rate constants for gaseous reactions of several electronically excited species J. Phys. Chem. Ref. Data 8 723-98

[407] Golde M F 1988 Reactions of $\mathrm{N}_{2}\left(A^{3} \sum_{\mathrm{u}}^{+}\right)$Int. J. Chem. Kinet. $2075-92$

[408] Herron J T 1999 Evaluated chemical kinetics data for reactions of $\mathrm{N}\left({ }^{2} D\right), \mathrm{N}\left({ }^{2} P\right)$ and $\mathrm{N}_{2}\left(A^{3} \sum_{\mathrm{u}}^{\mp}\right)$ in the gas phase Phys. Chem. Ref. Data 28 1453-83

[409] Copeland R A, Wise M L and Crosley D R 1988 Vibrational energy transfer and quenching of $\mathrm{OH}\left(A^{2} \sum^{+}, v^{\prime}=1\right)$ J. Phys. Chem. 92 5710-5

[410] Kienle R, Lee M P and Kohse-Hoinghaus K 1996 A scaling formalism for the representation of rotational energy transfer in $\mathrm{OH}\left(A^{2} \sum^{+}\right)$in combustion experiments Appl. Phys. B 63 403-18

[411] Velazco J E, Kolts J H and Setser D W 1978 Rate constants and quenching mechanisms for the metastable states of argon, krypton, and xenon J. Chem. Phys. 69 4357-73

[412] Smirnov B M 1974 Ions and Excited Atoms in Plasma (Moscow: Atomizdat)

[413] Capitelli M, Ferreira C M, Gordiets B F and Osipov A I 2000 Plasma Kinetics in Atmospheric Gases (Berlin: Springer)
[414] Itikawa Y 2007 Molecular Processes in Plasmas: Collisions of Charged Particles with Molecules (Berlin: Springer)

[415] Billing G D and Mikkelsen K V 1996 Introduction to Molecular Dynamics and Chemical Kinetics (New York: Wiley)

[416] Kochi J K 1973 Free Radicals (New York: Wiley)

[417] Alfassi Z B 1997 Peroxyl Radicals (New York: Wiley)

[418] Mozumder A 1999 Fundamentals of Radiation Chemistry (New York: Academic)

[419] NIST Standard Reference Data 2011 http://webbook.nist. gov/chemistry/fluid/

[420] Atkinson R A 1987 A structure-activity relationship for the estimation of rate constants for the gas-phase reactions of $\mathrm{OH}$ radicals with organic-compounds Int. J. Chem. Kinet. 19 799-828

[421] King M D, Canosa-Mas C E and Wayne R P 1999 A structure-activity relationship (SAR) for predicting rate constants for the reaction of $\mathrm{NO}_{3}, \mathrm{OH}$ and $\mathrm{O}_{3}$ with monoalkenes and conjugated dienes Phys. Chem. Chem. Phys. 12239-46

[422] Kwok E S C and Atkinson R 1995 Estimation of hydroxyl radical reaction rate constants for gas-phase organic compounds using a structure-reactivity relationship: an update Atmos. Environ. 29 1685-95

[423] US Environmental Protection Agency 2000 Predictive models and tools for assessing chemicals under the toxic substances control act (TSCA) www.epa.gov/oppt/ exposure/pubs/episuite.htm

[424] Minakata D and Crittenden J 2011 Linear free energy relationships between aqueous phase hydroxyl radical $\left(\mathrm{HO}^{\bullet}\right)$ reaction rate constants and free energy of activation Environ. Sci. Technol. 45 3479-56

[425] Minakata D, Song W and Crittenden J 2011 Reactivity of aqueous phase hydroxyl radical with halogenated carboxylate anions: experimental and theoretical studies Environ. Sci. Technol. 45 6057-65

[426] White R D et al 2014 Low-energy electron and positron transport in gases and soft-condensed systems of biological relevance Appl. Radiat. Isot. 83 77-85

[427] White R D and Robson R E 2011 Multiterm solution of a generalized Boltzmann kinetic equation for electron and positron transport in structured and soft condensed matter Phys. Rev. E 84031125

[428] White R D, Brunger M J, Garland N A, Robson R E, Ness K F, Garcia G, De Urquijo J, Dujko S and Petrovic Z L 2014 Electron swarm transport in THF and water mixtures Eur. Phys. J. D 68125

[429] Niemi K, Reuter S, Graham L M, Waskoenig J, Knake N, Schulz-von der Gathen V and Gans T 2010 Diagnostic based modelling of radio-frequency driven atmospheric pressure plasmas J. Phys. D: Appl. Phys. 43 124001-6

[430] Verreycken T, van der Horst R M, van Veldhuizen E M, Sadeghi N and Bruggeman P J 2013 Absolute calibration of $\mathrm{OH}$ density produced by a nanosecond pulsed filamentary discharge in atmospheric pressure $\mathrm{He}-\mathrm{H}_{2} \mathrm{O}$ : comparison of independent calibration methods $J$. Phys. D: Appl. Phys. 46 464004-14

[431] Kienle R, Lee M P and Kohse-Hoinghaus K 1996 A detailed rate equation model for the simulation of energy transfer in OH laser-induced fluorescence J. Appl. Phys. B 62 583-99

[432] Kohsehoinghaus K 1994 Laser techniques for the quantitative detection of reactive intermediates in combustion systems Prog. Energy Combust. Sci. 20 203-79

[433] Bruggeman P J and Brandenburg R 2013 Atmospheric pressure discharge filaments and microplasmas: physics, chemistry and diagnostics J. Phys. D: Appl. Phys. 46 464001-28 
[434] Dilecce G 2014 Optical spectroscopy diagnostics of discharges at atmospheric pressure Plasma Source Sci. Technol. 23051011

[435] Reuter S, Sousa J S, Stancu G D and van Helden J P 2015 Review on VUV to MIR absorption spectroscopy of atmospheric pressure plasma jets Plasma Sources Sci. Technol. 24054001

[436] Bockle S, Kazenwadel J, Kunzelmann T, Shin D, Schulz C and Wolfrum J 2000 Simultaneous single-shot laserbased imaging of formaldehyde, $\mathrm{OH}$, and temperature in turbulent flames Proc. Combust. Inst. 28 279-86

[437] Benedikt J, Ellerweg D, Hecimovic A and von Kuedell A 2012 Quadrupol masspectrometry of reactive plasmas (review) J. Phys. D: Appl. Phys. 45403001

[438] Mendham J, Denney R C, Barnes J D and Thomas M J K 1999 Vogel's Textbook of Quantitative Chemical Analysis 6th edn (Englewood Cliffs, NJ: Prentice-Hall)

[439] Ropcke J, Davies P B, Lang N, Rousseau A and Welzel S 2012 Applications of quantum cascade lasers in plasma diagnostics: a review J. Phys. D: Appl. Phys. 45423001

[440] Schroder D, Bahre H, Knake N, Winter J, de los Arcos T and Schulz-von der Gathen V 2012 Influence of target surfaces on the atomic oxygen distribution in the effluent of a micro-scaled atmospheric pressure plasma jet Plasma Source Sci. Technol. 21024007

[441] Komuro A, Ono R and Oda T 2010 Kinetic model of vibrational relaxation in a humid-air pulsed corona discharge Plasma Source Sci. Technol. 19055004

[442] Paul P H 1994 A model for temperature-dependent collisional quenching of $\mathrm{OH}\left(A^{2} \Sigma^{+}\right) J$. Quant. Spectrosc. Radiat. Transfer 51 511-24

[443] Ries D, Dilecce G, Robert E, Ambrico P F, Dozias S and Pouvesle J-M 2014 LIF and fast imaging plasma jet characterization relevant for NTP biomedical applications J. Phys. D: Appl. Phys. 47275401

[444] Yonemori S and Ono R 2014 Flux of OH and O radicals onto a surface by an atmospheric-pressure helium plasma jet measured by laser-induced fluorescence J. Phys. D: Appl. Phys. 47125401

[445] Yagi I, Ono R, Oda T and Takaki K 2015 Two-dimensional LIF measurements of humidity and $\mathrm{OH}$ density resulting from evaporated water from a wet surface in plasma for medical use Plasma Source Sci. Technol. 24015002

[446] Zhang S, van Gessel A F M, Van Grootel S C and Bruggeman P J 2014 The effect of collisional quenching of the O 3p 3PJ state on the determination of the spatial distribution of the atomic oxygen density in an APPJ operating in ambient air by TALIF Plasma Source Sci. Technol. 23025012

[447] Nielsen T, Bormann F, Burrows M and Anderson P 1997 Picosecond laser-induced fluorescence measurement of rotational energy transfer of $\mathrm{OH} A^{2} \Sigma^{+}\left(v^{\prime}=2\right)$ in atmospheric pressure flames Appl. Opt. 36 7960-9

[448] Bredin J, Dedrick J, Niemi K, West A, Wagenaars E, Gans T and O'Connell D 2014 Picosecond-TALIF measurements of atomic oxygen in RF driven atmospheric pressure plasma jets Bulletin of the American Physical Society, 67th Annual Gaseous Electronics Conf. (Raleigh, North Carolina) (American Physical Society)

[449] Bruggeman P J, Schram D, Gonzalez M A, Rego R, Kong M G and Leys C 2009 Characterization of a direct dc-excited discharge in water by optical emission spectroscopy Plasma Source Sci. Technol. 18025017

[450] Andre P et al 2002 Theoretical study of column of discharge with liquid non-metallic (tap water) electrodes in air at atmospheric pressure J. Phys. D: Appl. Phys. 35 1846-54
[451] Miron C, Bratescu M A, Saito N and Takai O 2011 Optical diagnostic of bipolar electrical discharges in $\mathrm{HCl}, \mathrm{KCl}$, and KOH solutions J. Appl. Phys. 109123301

[452] Bruggeman P J and Schram D 2010 On OH production in water containing atmospheric pressure plasmas Plasma Source Sci. Technol. 19 045025-34

[453] Bruggeman P J, Iza F, Lauwers D, Kong M G, Aranda Gonzalvo Y, Leys C and Schram D 2010 Electronic quenching of $\mathrm{OH}(A)$ by water in atmospheric pressure plasmas and its influence on the gas temperature determination by $\mathrm{OH}(A-X)$ emission Plasma Source Sci. Technol. 19 015016-23

[454] Bruggeman P J, Cunge G and Sadeghi N 2012 Absolute OH density measurements by broad- band UV absorption in diffuse atmospheric-pressure $\mathrm{He}-\mathrm{H}_{2} \mathrm{O} \mathrm{RF}$ glow discharges Plasma Source Sci. Technol. 21 035019-25

[455] Bruggeman P J, Sadeghi N, Schram D and Linss V 2014 Gas temperature determination from rotational lines in nonequilibrium plasmas: a review Plasma Source Sci. Technol. 23 023001-32

[456] Suslick K S and Flannigan D J 2007 Inside a collapsing bubble: sonoluminescence and the conditions during cavitation Ann. Rev. Phys. Chem. 59 659-83

[457] Nikitenko S I 2014 Plasma formation during acoustic cavitation: toward a new paradigm for sonochemistry $A d v$. Phys. Chem. 2014 173878-86

[458] Flannigan D J and Suslick K S 2010 Inertially confined plasma in an imploding bubble Nat. Phys. 6 598-601

[459] Hamdan A, Marinov I, Rousseau A and Belmonte T 2014 Time-resolved imaging of nanosecond-pulsed microdischarges in heptane J. Phys. D: Appl. Phys. 47055203

[460] Wilbers A T M, Kroesen G M W, Timmermans C J and Schram D 1991 The continuum emission of an arc plasma J. Quantum Spectrosc. Radiat. Transfer 45 1-10

[461] Lavrov B P, Melnikov A S, Kaning M and Roepcke J 1999 UV continuum emission and diagnostics of hydrogencontaining nonequilibrium plasmas Phys. Rev. E 593526

[462] Shinfuku N, Toyota H, Mukasa S, Takahashi Y, Maehara T, Kawashima A and Yamashita H 2008 Discharge characteristcs of microwave and high-frequency in-liquid plasma in water Appl. Phys. Express 1046002

[463] Bernstein L and Zakin M R 1996 Cavitation thermometry using molecular and continuum sonoluminescence $J$. Phys. Chem. $1006612-9$

[464] Lavrov B P 2001 Novel mechanism for single bubble sonoluminescence arxiv:physics/0102060

[465] Grinenko A, Ya E, Krasik Y E, Efimov S, Fedotov A and Gurovich V T 2006 Nanosecond time scale, high power electrical wire explosion in water Phys. Plasma 13042701

[466] Dam J S and Levinsen M T 2004 Size of the light-emitting region in a sonoluminescing bubble Phys. Rev. Lett. 92144301

[467] Marinov I 2013 Plasmas in contact with liquids and at the interfaces. Application for living cell treatment $P h D$ Thesis, Ecole Polytechnique

[468] Xu D A, Lacoste D A, Rusterholtz D L, Elias P Q, Stancu G D and Laux C O 2011 Experimental study of the hydrodynamic expansion following a nanosecond repetitively pulsed discharge in air Appl. Phys. Lett. 99121502

[469] Allen M G, McManus K R, Sonnenfroh D M and Paul P H 1995 Planar-laser-induced-fluorescence imaging measurements of $\mathrm{OH}$ and hydrocarbon fuel fragments in high-pressure spray-flame combustion Appl. Opt. 34 6287-300

[470] Locke R J, Hicks Y R, Anderson R C and Zaller M M 1998 Optical fuel injector patternation measurements in advanced liquid-fueled, high pressure, gas turbine combustors Combust. Sci. Technol. 138 297-311 
[471] Bachalo W D and Houser M J 1984 Phase Doppler spray analyzer for simultaneous measurements of drop size and velocity distributions $O p t$. Eng. 23 583-90

[472] Graves D B 2012 The emerging role of reactive oxygen and nitrogen species in redox biology and some implications for plasma applications to medicine and biology J. Phys. D: Appl. Phys. 45263001

[473] Sen C K and Roy S 2008 Redox signals in wound healing Biochim. Biophys. Acta 1780 1348-61

[474] Sen C K 2009 Wound healing essentials: let there be oxygen Wound Rep. 17 1-18

[475] Schuring J 2000 Redox: Fundamentals, Processes and Applications (Berlin: Springer)

[476] Bourbonnais R, Leech D and Paice M G 1998 Electrochemical analysis of the interactions of laccase mediators with lignin model compounds Biochim. Biophys. Acta 1379 381-90

[477] Buehler R E, Staehelin J and Hoigne J 1984 Ozone decomposition in water studied by pulsed radiolysis. 1 $\mathrm{HO}_{2} / \mathrm{O}_{2}^{-}$and $\mathrm{HO}_{3} / \mathrm{O}_{3}^{-}$as intermediates J. Phys. Chem. $882560-4$

[478] Moore K and Roberts L J 1998 Measurement of lipid peroxidation Free Radic. Res. 28 659-71

[479] Chance B, Greenstein D S and Roughton F J W 1952 The mechanism of catalase action.1. Steady-state analysis Arch. Biochem. Biophys. 37 301-21

[480] Desesso J M, Scialli A R and Goeringer G C 1994 D-mannitol, a specific hydroxyl free radical scavenger, reduces the developmental toxicity of hydroxyurea in rabbits Teratology 49 248-59

[481] Pfeiffer S, Leopold E, Hemmens B, Schmidt K, Werner E R and Mayer B 1997 Interference of carboxy-PTIO with nitric oxide- and peroxynitrite-mediated reactions Free Radic. Biol. Med. 22 787-94

[482] Tresp H, Hammer M U, Winter J, Weltmann K D and Reuter S 2013 Quantitative detection of plasma-generated radicals in liquids by electron paramagnetic resonance spectroscopy J. Phys. D: Appl. Phys. 46435401

[483] Tresp H, Hammer M U, Weltmann K D and Reuter S 2014 Effects of atmosphere composition and liquid type on plasma generated reactive species in biologically relevant solutions Plasma Med. 3 45-55

[484] Eisenberg G M 1943 Colorimetric determination of hydrogen peroxide Ind. Eng. Chem. Anal. Ed. 15 449-56

[485] Bader H and Hoigne J 1981 Determination of ozone in water by the indigo method Water Res. 15 449-56

[486] Fox J B 1979 Kinetics and mechanisms of the griess reaction Anal. Chem. 51 1493-502

[487] Wardman P 2007 Fluorescent and luminescent probes for measurement of oxidative and nitrosative species in cells and tissues: progress, pitfalls, and prospects Free Radic. Biol. Med. 43 995-1022

[488] Lukes P, Dolezalova E, Sisrova I and Clupek M 2014 Aqueous-phase chemistry and bactericidal effects from an air discharge plasma in contact with water: evidence for the formation of peroxynitrite through a pseudo-secondorder post-discharge reaction of $\mathrm{H}_{2} \mathrm{O}_{2}$ and $\mathrm{HNO}_{2}$ Plasma Source Sci. Technol. 23015019

[489] Sahni M and Locke B R 2006 Quantification of hydroxyl radicals produced in aqueous phase pulsed electrical discharge reactors Ind. Eng. Chem. Res. 45 5819-25

[490] Sahni M and Locke B R 2006 Quantification of reductive species produced by high voltage electrical discharges in water Plasma Proc. Polym. 3 342-54

[491] Kirkpatrick M J and Locke B R 2006 Effects of platinum electrode on hydrogen, oxygen, and hydrogen peroxide formation in aqueous phase pulsed corona electrical discharge Ind. Eng. Chem. Res. 45 2138-42
[492] Walling C 1975 Fentons reagent revisited Acc. Chem. Res. $8125-31$

[493] Sharma A K, Locke B R, Arce P and Finey W C 1993 A preliminary-study of pulsed streamer corona discharge for the degradation of phenol in aqueous-solutions Hazard. Waste Hazard. Mater. 10 209-19

[494] Lukes P, Clupek M, Babicky V, Sisrova I and Janda V 2011 The catalytic role of tungsten electrode material in the plasmachemical activity of a pulsed corona discharge in water Plasma Source Sci. Technol. 20034011

[495] Anbar M and Taube H 1954 Interaction of nitrous acid with hydrogen peroxide and with water J. Am. Chem. Soc. 76 6243-7

[496] Hoigne J and Bader H 1976 Role of hydroxyl radical reactions in ozonation processes in aqueous solutions Water Res. 10 377-86

[497] Staehelin J and Hoigne J 1982 Decomposition of ozone in water-rate of initiation by hydroxide ions and hydrogen peroxide Environ. Sci. Technol. 16 676-81

[498] Graf E and Penniston J T 1980 Method for determination of hydrogen-peroxide, with its application illustrated by glucose assay Clin. Chem. 26 658-60

[499] Nogueira R F P, Oliveira M C and Paterlini W C 2005 Simple and fast spectrophotometric determination of $\mathrm{H}_{2} \mathrm{O}_{2}$ in photo-Fenton reactions using metavanadate Talanta 66 86-91

[500] Park J Y and Lee Y N 1988 Solubility and decomposition kinetics of nitrous acid in aqueous solution J. Phys. Chem. 92 6294-302

[501] Oehmigen K, Winter J, Hahnel M, Wilke C, Brandenburg R, Weltmann K D and von Woedtke T 2011 Estimation of possible mechanisms of Escherichia coli inactivation by plasma treated sodium chloride solution Plasma Proc. Polym. 8 904-13

[502] Traylor M J, Pavlovich M J, Karim S, Hait P, Sakiyama Y, Clark D S and Graves D B 2011 Long-term antibacterial efficacy of air plasma-activated water J. Phys. D: Appl. Phys. 44472001

[503] Machala Z, Tarabova B, Hensel K, Spetlikova E, Sikurova L and Lukes P 2013 Formation of ROS and RNS in water electro-sprayed through transient spark discharge in air and their bactericidal effects Plasma Proc. Polym. 10 649-59

[504] Naitali M, Kamgang-Youbi G, Herry J M, Bellon-Fontaine M N and Brisset J L 2010 Combined effects of long-living chemical species during microbial inactivation using atmospheric plasma-treated water $\mathrm{Appl}$. Environ. Microbiol. 76 7662-4

[505] Goldstein S, Lind J and Merenyi G 2005 Chemistry of peroxynitrites as compared to peroxynitrates Chem. Rev. 105 2457-70

[506] Pryor W A 1986 Oxyradicals and related species-their formation, lifetimes, and reactions Ann. Rev. Physiol. 48 657-67

[507] Janzen E G and Blackburn B J 1968 Detection and identification of short-lived free radicals by an electron spin resonance trapping technique J. Am. Chem. Soc. 90 5909-10

[508] Rosen G M 1999 Free Radicals: Biology and Detection by Spin Trapping (New York: Oxford University Press)

[509] Lagercrantz C 1971 Spin trapping of some short-lived radicals by the nitroxide method J. Phys. Chem. $753466-75$

[510] Janzen E G, Kotake Y and Hinton R D 1992 Stabilities of hydroxyl radical spin adducts of PBN-type spin traps Free Radic. Biol. Med. 12 167-73

[511] Han J Y, Hong J T, Nam S Y and Oh K W 2012 Evaluation of hydroxyl radical reduction activity of red ginseng extract using ESR spectroscopy J. Biomed. Res. 13 83-92 
[512] Nakamura K, Kanno T, Ikai H, Sato E, Mokudai T, Niwano Y, Ozawa T and Kohno M 2010 Reevaluation of quantitative ESR spin trapping analysis of hydroxyl radical by applying sonolysis of water as a model system Bull. Chem. Soc. Japan 83 1037-46

[513] Villamena F A and Zweier J L 2002 Superoxide radical trapping and spin adduct decay of 5-tert-butoxycarbonyl5-methyl-1-pyrroline N-oxide (BocMPO): kinetics and theoretical analysis J. Chem. Soc., Perkin Trans. 2 7 1340-4

[514] Khan N, Wilmot C M, Rosen G M, Demidenko E, Sun J, Joseph J, O'Hara J, Kalyanaraman B and Swartz H M 2003 Spin traps: in vitro toxicity and stability of radical adducts Free Radic. Biol. Med. 34 1473-81

[515] Gilbert B C, Davies M J and Murphy D M Electron Paramagnetic Resonance (Cambridge: Royal Society of Chemistry)

[516] Chalier F and Tordo P 2002 Diisopropoxyphosphoryl-5methyl-1-pyrroline N-oxide, DIPPMPO, a crystalline analog of the nitrone DEPMPO: synthesis and spin trapping properties J. Chem. Soc., Perkin Trans. 2 $122110-7$

[517] Kamibayashi M, Oowada S, Kameda H, Okada T, Inanami O, Ohta S, Ozawa T, Makino K and Kotake Y 2006 Synthesis and characterization of a practically better DEPMPO-type spin trap, 5-(2,2-dimethyl-1,3-propoxy cyclophosphoryl)5-methyl-1-pyrroline N-oxide (CYPMPO) Free Radic. Res. 40 1166-72

[518] Rosen G M, Tsai P, Weaver J, Porasuphatana S, Roman L J, Starkov A A, Fiskum G and Pou S 2002 The role of tetrahydrobiopterin in the regulation of neuronal nitricoxide synthase-generated superoxide J. Biol. Chem. 277 40275-80

[519] Tsai P, Ichikawa K, Mailer C, Pou S, Halpern H J, Robinson B H, Nielsen R and Rosen G M 2003 Esters of 5-carboxyl-5-methyl-1-pyrroline $\mathrm{N}$-oxide: a family of spin traps for superoxide J. Org. Chem. $\mathbf{6 8} 7811-7$

[520] Bullock A T, Gavin D L and Ingram M D 1980 Electron-spin resonance detection of spin-trapped radicals formed during the glow-discharge electrolysis of aqueous-solutions J. Chem. Soc., Faraday Trans. 76 648-53

[521] Hase $\mathrm{H}$ and Harada K 2001 ESR detection of $\mathrm{OH}$ and $\mathrm{H}$ generated by contact glow discharge in aqueous solution Viva Origino 29 61-2

[522] Bai N et al 2011 Inactivation of staphylococcus aureus in water by a cold, $\mathrm{He} / \mathrm{O}_{2}$ atmospheric pressure plasma microjet Plasma Proc. Polym. 8 424-31

[523] Tani A, Ono Y, Fukui S, Ikawa S and Kitano K 2012 Free radicals induced in aqueous solution by non-contact atmospheric-pressure cold plasma Appl. Phys. Lett. 100254103

[524] Sun P, Sun Y, Wu H, Zhu W, Lopez J L, Liu W, Zhang J, Li R and Fang J 2011 Atmospheric pressure cold plasma as an antifungal therapy Appl. Phys. Lett. 98021501

[525] Zhang Q, Sun P, Feng H, Wang R, Laing Y, Zhu W, Becker K H, Zhang J and Fang J 2012 Assessment of the roles of various inactivation agents in an argon-based direct current atmospheric pressure cold plasma jet J. Appl. Phys. 111123305

[526] Wu H Y et al 2012 Reactive oxygen species in a non-thermal plasma microjet and water system: generation, conversion, and contributions to bacteria inactivation-an analysis by electron spin resonance spectroscopy Plasma Proc. Polym. 9 417-24

[527] Halliwell B, Gutteridge J M and Aruoma O I 1987 The deoxyribose method: a simple 'test-tube' assay for determination of rate constants for reactions of hydroxyl radicals Anal. Biochem. $165215-9$

[528] Bruggeman P J and Fiddy S 2015 Unpublished data
[529] Bastidas-Oyanedela J R, Mohd-Zakib Z, Prattb S, Steyera J P and Batstoneb D J 2010 Development of membrane inlet mass spectrometry for examination of fermentation processes Talanta 83 482-92

[530] Lloyd D and Scott R I 1983 Direct measurement of dissolved gases in microbiological systems using membrane inlet mass spectrometry J. Microbiol. Methods $1313-28$

[531] Marotta E, Schiorlin M, Ren X, Rea M and Paradisi C 2011 Advanced oxidation process for degradation of aqueous phenol in a dielectric barrier discharge reactor Plasma Proc. Polym. 8 867-75

[532] Zazo J A, Casas J A, Mohedano A F, Gilarranz M A and Rodriguez J J 2005 Chemical pathway and kinetics of phenol oxidation by Fenton's reagent Environ. Sci. Technol. 39 9295-302

[533] Hoeben W F L M, van Veldhuizen E M, Rutgers W R, Cramers C A M G and Kroesen G M W 2000

The degradation of aqueous phenol solutions by pulsed positive corona discharges Plasma Source Sci. Technol. 9 361-9

[534] Magureanu M, Dobrin D, Mandache N B, Bradu C, Medvedovici A and Parvulescu V I 2013 The mechanism of plasma destruction of enalapril and related metabolites in water Plasma Proc. Polym. 10 459-68

[535] McDowell D C, Huber M M, Wagner M, Von Gunten U and Ternes T A 2005 Ozonation of carbamazepine in drinking water: identification and kinetic study of major oxidation products Environ. Sci. Technol. 398014

[536] Poerschmann J and Trommler U 2009 Pathways of advanced oxidation of phenol by Fenton's reagent-identification of oxidative coupling intermediates by extractive acetylation J. Chromatogr. A 12165570

[537] Wandell R J, Bresch S, Hsieh K, Alabugin I V and Locke B R 2014 Formation of alcohols and carbonyl compounds from hexane and water in a liquid film plasma reactor IEEE Trans. Plasma Sci. 42 1195-205

[538] Castello-Beltran C, Palmer E A, Buckley B R and Iza F 2015 Virtues and limitations of Pittsburg green for ozone detection Chem. Commun. 15 1579-82

[539] Halliwell B and Gutteridge J M 2007 Free Radicals in Biology and Medicine (Oxford: Oxford University Press)

[540] Kalyanaraman B et al 2012 Measuring reactive oxygen and nitrogen species with fluorescent probes: challenges and limitations Free Radic. Biol. Med. 52 1-6

[541] Davidovits P, Kolb C E, Williams L R, Jayne J T and Worsnop D R 2006 Mass accommodation and chemical reactions at gas-liquid interfaces Chem. Rev. 106 1323-54

[542] Donaldson D J and Vaida V 2006 The influence of organic films at the air-aqueous boundary on atmospheric processes Chem. Rev. 106 1445-61

[543] Ruehl C R, Nah T, Isaacman G, Worton D R, Chan A W H, Kolesar K R and Wilson K R 2013 The influence of molecular structure and aerosol phase on the heterogeneous oxidation of normal and branched alkanes by OH J. Phys. Chem. 117 3990-4000

[544] Dennis-Smither B J, Miles R E H and Reid J P 2012 Oxidative aging of mixed oleic acid/sodium chloride aerosol particles J. Geophys. Res. 117 D20204

[545] Miles R E H, Knox K J, Reid J P, Laurain A M C and Mitchem L 2010 Measurements of mass and heat transfer at a liquid water surface during condensation or evaporation of a sub-nanometre thickness layer of water Phys. Rev. Lett. 105116101

[546] Davies J F, Miles R E H, Haddrell A E and Reid J P 2013 Influence of organic films on the evaporation and condensation of water in aerosol Proc. Natl Acad. Sci. USA $1108807-12$ 
[547] Smith J D, Cappa C D, Drisdell W S, Cohen R C and Saykally R J 2006 Raman thermometry measurements of free evaporation from liquid water droplets J. Am. Chem. Soc. 128 12892-8

[548] Enami S, Sakamoto Y and Colussi A J 2014 Fenton chemistry at aqueous interfaces Proc. Nat. Acad. Sci. USA $111623-8$

[549] Mmereki B T, Donaldson D J, Gilman J B, Eliason T L and Vaida V 2004 Kinetics and products of the reaction of gasphase ozone with anthracene adsorbed at the air-aqueous interface Atmos. Environ. 38 6091-103

[550] Corn R M and Higgins D A 1994 Optical second harmonic generation as a probe of surface chemistry Chem. Rev. 94 107-25

[551] Kondo T and Ito T 2014 Flipping water molecules at insulator/solution interface using an externally applied weak electric field Appl. Phys. Lett. 104101601

[552] Ghosal S, Hemminger J C, Bluhm H, Mun B S, Hebenstreit E L D, Ketteler G and Salmeron M 2005 Electron spectroscopy of aqueous solution interfaces reveals surface enhancement of halides Science 307 563-6

[553] Margarella A M, Perrine K A, Leweis T, Faubel M, Winter B and Hemminger J C 2013 Dissociation of sulfuric acid in aqueous solution: determination of the photoelectron spectral fingerprints of $\mathrm{H}_{2} \mathrm{SO}_{4}, \mathrm{HSO}_{4}^{-}$, and $\mathrm{SO}_{4}^{2-}$ in Water J. Phys. Chem. C 117 8131-7

[554] Pruyne G, Lee M, Fa C, Redondo A B, Kleibert A, Ammann M and Krisch M J 2014 Liquid-vapor interface of formic acid solutions in salt water : a comparison of macroscopic surface tension and microscopic in situ $\mathrm{x}$-ray photoelectron spectroscopy measurements $J$. Phys. Chem. C 118 29350-60

[555] Voss L F, Bazerbashi M F, Beekman C P, Hadad C M and Allen H C 2007 Oxidation of oleic acid at air/liquid interfaces J. Geophys. Res. 112 D06209

[556] Nathanson G M 2004 Molecular beam studies of gas-liquid interfaces Ann. Rev. Phys. Chem. 55 231-55

[557] Alexander W, Zhang J, Murray V J, Nathanson G M and Minton T K 2012 Kinematics and dynamics of atomicbeam scattering on liquid and self-assembled monolayer surfaces Faraday Discuss. 157355

[558] King K L, Paterson G, Rossi G E, Iljina M, Westacott R E, Costen M L and McKendrick K G 2013 Inelastic scattering of $\mathrm{OH}$ radicals from organic liquids: isolating the thermal desorption channel Phys. Chem. Chem. Phys. 15 12852-63

[559] Tesa-Serrate M, King K L, Paterson G, Costen M L and McKendrick K G 2014 Site and bond-specific dynamics of reactions at the gas-liquid interface Phys. Chem. Chem. Phys. 16 173-83

[560] Bockris J O, Conway B E and White R E 2002 Modern Aspects of Electrochemistry (Dordrecht: Kluwer)

[561] Bruggeman P J, Van Slycken J, Degroote J, Vierendeels J, Verleysen P and Leys C 2008 DC electrical breakdown in a metal pin-water electrode system IEEE Trans. Plasma Sci. 36 1138-9

[562] Sugimoto T, Asano K and Higashiyama Y 2001 Negative corona discharge at a tip of water cone deformed under $\mathrm{dc}$ field $J$. Electrost. 53 25-38

[563] Shirai N, Ichinose K, Uchida S and Tochikubo F 2011 Influence of liquid temperature on the characteristics of an atmospheric dc glow discharge using a liquid electrode with a miniature helium flow Plasma Source Sci. Technol. 20034013

[564] Zhang S, Sobota A, van Veldhuizen E M and Bruggeman P J 2015 Gas flow characteristics of a time modulated APPJ: the effect of gas heating on flow dynamics J. Phys. D: Appl. Phys. 48015203
[565] Kanazawa S, Kawano H, Watanabe S, Furuki T, Akamine S, Ichiki R, Ohkubo T, Kocik M and Mizeraczyk J 2011 Observation of $\mathrm{OH}$ radicals produced by pulsed discharges on the surface of liquid Plasma Source Sci. Technol. 200304010

[566] Fernandez Rivas D, Prosperetti A, Zijlstra A G, Lohse D and Gardeniers H J G E 2010 Efficient Sonochemistry through Microbubbles Generated with Micromachined Surfaces Angew. Chem. Int. Ed. 49 9699-701

[567] Rumbach P, Griggs N, Sankaran R M and Go D B 2014 Visualization of electrolytic reactions at a plasma-liquid interface IEEE Trans. Plasma Sci. 42 2610-1

[568] Djakaou I-S, Ghezzar R M, Zekri M E-M, Abdelmalek F, Cavadias S and Ognier S 2015 Removal of model pollutants in aqueous solution by gliding arc discharge. Part II: Modeling and simulation study Plasma Chem. Plasma Process. 35 143-57

[569] Marenich A V, Cramer C J and Truhlar D G 2009 Universal solvation model based on solute electron density and on a continuum model of the solvent defined by the bulk dielectric constant and atomic surface tensions J. Phys. Chem. B 113 6378-96

[570] Takeuchi K, Ishii Y and Yasuka K 2012 Modelling chemical reactions in dc plasma inside oxygen bubbles in water Plasma Source Sci. Technol. 21015006

[571] Marinov I, Guaitella O, Rousseau A and Starikovskaia S M 2013 Cavitation in the vicinity of the high-voltage electrode as a key step of nanosecond breakdown in liquids Plasma Source Sci. Technol. 22042001

[572] Pekker M, Seepersad Y, Shneider M N, Fridman A and Dobrynin D 2014 Initiation stage of nanosecond breakdown in liquid J. Phys. D: Appl. Phys. 47025502

[573] Starikovskiy A 2013 Pulsed nanosecond discharge development in liquids with various dielectric permittivity constants Plasma Source Sci. Technol. 22012001

[574] Joshi R P, Qian J, Zhao G, Kolb J, Schoenbach K H, Schamiloglu E and Gaudet J 2004 Are microbubbles necessary for the breakdown of liquid water subjected to a submicrosecond pulse? J. Appl. Phys. 96 5129-39

[575] Hamaguchi S 2012 Chemically reactive species in liquids generated by atmospheric pressure plasmas and their roles in plasma medicine AIP Conf. Proc. 8th Int. Conf. on Atomic and Molecular Data and Their Applications ICAMDATA-2012 (Gaithersburg, Maryland) ed J D Gillaspy et al (AIP Publishing) pp 214-22

[576] Elliot A J, McCracken D R, Buxton G V and Wood N D 1990 Estimation of rate constants for near-diffusioncontrolled reactions in water at high temperatures J. Chem. Soc. Faraday Trans. 86 1539-47

[577] Turner M M 2015 Uncertainty and sensitivity analysis in complex plasma chemistry models Plasma Source Sci. Technol. 25015003

[578] Islam M M, Alam M T and Ohsaka T 2008 Electrical doublelayer structure in ionic liquids: a corroboration of the theoretical model by experimental results J. Phys. Chem. C 112 16568-1657

[579] Shirafuji T, Nakamura A and Tochikubo F 2014 Numerical simulation of electric double layer in contact with dielectric barrier discharge: effects of ion transport parameters in liquid Japan. J. Appl. Phys. 53 03DG4

[580] Givi P 1989 Model free simulations of turbulent reactive flows Prog. Energy Combust. Sci. 15 1-107

[581] Pope S B 2000 Turbulent Flows (Cambridge: Cambridge University Press)

[582] Scardovelli R and Zaleski S 1999 Direct numerical simulation of free-surface and interfacial flow Ann. Rev. Fluid Mech. 31 567-603 
[583] Rodi W 1997 Comparison of LES and RANS calculations of the flow around bluff bodies J. Wind Eng. Ind. Aerodyn. 69 55-75

[584] Germano M 1992 Turbulence: the filtering approach J. Fluid Mech. 238 325-36

[585] Liepmann D and Gharib M 1992 The role of streamwise vorticity in the near field entrainment of round jets $J$. Fluid Mech. 245 643-68

[586] Ottino J M 1989 The Kinematics of Mixing: Stretching, Chaos and Transport (Cambridge: Cambridge University Press)

[587] Kerstein A R 1992 Linear-eddy modeling of turbulent transport. Part 7. Finite-rate chemistry and multi-stream mixing J. Fluid Mech. 240 289-313

[588] Fox R O 2003 Computational Models for Turbulent Reacting Flows (Cambridge: Cambridge University Press)

[589] Givi P 2006 Filtered density function of subgrid scale modeling of turbulent combustion AIAA J. 44 16-23

[590] Shigeta M 2013 Three-dimensional flow dynamics of an argon RF plasma with dc jet assistance: a numerical study J. Phys. D: Appl. Phys. 46015401

[591] Trelles J P, Chazelas C, Vardelle A and Heberlein J V R 2009 Arc plasma torch modeling J. Therm. Spray Technol. 18728

[592] Zhou Q, Li H, Xu X, Liu F, Guo S, Chang X, Guo W and Xu P 2009 Comparative Study of turbulence modles on highly constricted plasma cutting arc J. Phys. D: Appl. Phys. 42015210

[593] Unverdi S and Tryggvason G 1992 A front-tracking method for viscous, incompressible, multi-fluid flows J. Comput. Phys. 100 25-37

[594] Tryggvason G, Bunner B, Esmaeeli A, Juric D, Al-Rawahi N, Tauber W and Han J 2001 A front-tracking method for the computations of multiphase flow J. Comput. Phys. $169708-59$

[595] Hirt C and Nichols B 1981 Volume of fluid (VOF) method for the dynamics of free boundaries J. Comput. Phys. 39 201-25

[596] Mulder W, Osher S and Sethian J 1992 Computing interface motion in compressible gas dynamics J. Comput. Phys. 100 209-28

[597] Sussman M, Smereka P and Osher S 1994 A level set approach for computing solutions to incompressible two-phase flow J. Comput. Phys. 114 146-59

[598] Yecko P and Zaleski S 2005 Transient growth in two-phase mixing layers J. Fluid Mech. 528 43-52

[599] Wang L L, Wang H P, Lu F G, Murphy A B and Carlson B E 2014 Numerical analysis of Al vapour effects in gas metal arc welding of Al alloys Sci. Technol. Weld. Joining $19361-8$

[600] Garrick S C 2016 Unpublished data

[601] Liu M B and Liu G R 2010 Smoothed particle hydrodynamics $(\mathrm{SPH})$ : an overview and recent developments Arch. Comput. Methods Eng. 17 25-76

[602] Bluhm H 2006 Pulsed Power Systems: Principles and Applications (Berlin: Springer)

[603] Kogelschatz U 1998 Process Technologies for Water Treatment ed S Stucki (New York: Plenum) pp 87-118

[604] Marinov D and Braithwaite N S J 2014 Power coupling and electrical characterization of a radio-frequency micro atmospheric pressure plasma jet Plasma Source Sci. Technol. 23062005

[605] Hofmann S, van Gessel A F M, Verreycken T and Bruggeman P J 2011 Power dissipation, gas temperature and electron density of a cold atmospheric pressure helium and argon RF plasma jet Plasma Source Sci. Technol. 20 065010-22

[606] Yang X, Moravej M, Nowling G R, Babayan S E, Panelon J, Chang J P and Hicks R F 2005 Comparison of an atmospheric pressure, radio-frequency discharge operating in the $\alpha$ and $\gamma$ modes Plasma Source Sci. Technol. $14314-20$

[607] Ivkovic M, Jovicevic S and Konjevic N 2004 Low electron density diagnostics: development of optical emission spectroscopic techniques and some applications to microwave induced plasmas Spectrochim. Acta B 59 591-605

[608] Muraoka K and Kono A 2011 Laser Thomson scattering for low-temperature plasmas J. Phys. D: Appl. Phys. 44043001

[609] Warner K and Hieftje G M 2002 Thomson scattering from analytical plasmas Spectrochim. Acta B 57 201-41

[610] van Gessel A F M, Carbone E A D, Bruggeman P J and van der Mullen J J A M 2012 Laser scattering on an atmospheric pressure plasma jet: disentangling Rayleigh, Raman and Thomson scattering Plasma Source Sci. Technol. 21015003

[611] Ito Y, Sakai O and Tachibana K 2010 Measurement of electron density in a microdischarge integrated device operated in nitrogen at atmospheric pressure using a millimeter-wave transmission method Plasma Source Sci. Technol. 19025006

[612] Park S, Choe W, Youn Moon S and Park J 2014 Electron density and temperature measurement by continuum radiation emitted from weakly ionized atmospheric pressure plasmas Appl. Phys. Lett. 104084103

[613] Taghizadeh L, Nikiforov A Y, Morent R, van der Mullen J J A M and Leys C 2014 Determination of the electron temperature of atmospheric pressure argon plasmas by absolute line intensities and a collisional radiative model Plasma Proc. Polym. 11 777-86

[614] Schafer J, Foest R, Reuter S, Kewitz T, Sperka J and Weltmann K D 2012 Laser schlieren deflectometry for temperature analysis of filamentary non-thermal atmospheric pressure plasma Rev. Sci. Instrum. 83103506

[615] Starikovskaia S M, Allegraud K, Guaitella O and Rousseau A 2010 On electric field measurements in surface dielectric barrier discharge J. Phys. D: Appl. Phys. 43124007

[616] Ito T, Kobayashi K, Mueller S, Luggenholscher D, Czarnetzki U and Hamaguchi S 2009 Electric field measurements in an atmospheric or higher pressure gas by coherent Raman scattering of nitrogen J. Phys. D: Appl. Phys. 42092003

[617] Arroyo M P and Hanson R K 1993 Absorption measurements of water-vapor concentration, temperature, and line-shape parameters using a tunable InGaAsP diode laser Appl. Opt. 32 6104-16

[618] Penney C M and Lapp M 1976 Raman-scattering cross sections for water vapor J. Opt. Soc. Am. 66 422-5

[619] Dunnbier M, Schmidt-Bleker A, Winter J, Wolfram M, Hippler R, Weltmann K D and Reuter S 2013 Ambient air particle transport into the effluent of a cold atmosphericpressure argon plasma jet investigated by molecular beam mass spectrometry J. Phys. D: Appl. Phys. 46435203

[620] Dobele H F, Mosbach T, Niemi K and Schulz-von der Gathen V 2005 Laser induced fluorescence measurements of absolute atomic densities: concepts and limitations Plasma Source Sci. Technol. 14 S31-41

[621] Tachibana K 2002 VUV to UV laser spectroscopy of atomic species in processing plasmas Plasma Source Sci. Technol. 11 A166-72

[622] van Gessel A F M, Alards K M J and Bruggeman P J 2013 NO production of an atmospheric pressure RF plasma jet J. Phys. D: Appl. Phys. 46265202

[623] Nemschokmichal S and Meichsner J $2013 \mathrm{~N}_{2}\left(A^{3} \sum_{\mathrm{u}}^{+}\right)$ metastable density in nitrogen barrier discharges: I LIF diagnostics and absolute calibration by Rayleigh scattering Plasma Source Sci. Technol. 22015005 
[624] Nierman B, Boeke M, Sadeghi N and Winter J 2010 Space resolved density measurements of argon and helium metastable atoms in radio-frequency generated $\mathrm{He}-\mathrm{Ar}$ microplasmas Eur. Phys. J. D 60 489-95

[625] Belostotskiy S G, Donnelly V M, Economou D J and Sadeghi N 2009 Spatially resolved measurments of argon metastable $\left(1 s_{5}\right)$ density in a high pressure microdischarge using diode laser absorption spectroscopy IEEE Trans. Plasma Sci. 37 852-8

[626] Stancu G D, Janda M, Kaddouri F, Lacoste D A and Laux C O 2010 Time-resolved CRDS measurements of the density produced by nanosecond discharges in atmospheric pressure nitrogen and air J. Phys. Chem. A $114201-8$

[627] Sakiyama Y, Graves D B, Chang H W, Shimizu T and Morfill G E 2012 Plasma chemistry model of surface microdischarge in humid air and dynamics of reactive neutral species J. Phys. D: Appl. Phys. 45425201

[628] Winter J et al 2014 Tracking plasma generated $\mathrm{H}_{2} \mathrm{O}_{2}$ from gas into liquid phase and revealing its dominant impact on human skin cells J. Phys. D: Appl. Phys. 47285401

[629] Bruggeman P J, Iza F, Lauwers D and Aranda Gonzalvo Y 2010 Mass spectrometry study of positive and negative ions in a capacitively coupled atmospheric pressure RF excited glow discharge in He-water mixtures $J$. Phys. D: Appl. Phys. 43 012003-9

[630] Bieber T, Glad X, de Poucques L, Hugon R, Vasseur J L and Bougdira J 2013 Argon ion and neutral metastable levels destruction in a magnetically enhanced inductively coupled plasma reactor Open Plasma Phys. J. 6 32-43

[631] Keiichiro U, Ito Y, Tachibana K and Ganguly B N 2008 Behavior of $\mathrm{N}_{2}^{+}$ions in $\mathrm{He}$ microplasma jet at atmospheric pressure measured by laser induced fluorescence spectroscopy Appl. Phys. Express 1066004

[632] Ahn T H, Nakamura K and Sugai H 1996 Negative ion measurements and etching in a pulsed-power inductively coupled plasma in chlorine Plasma Source Sci. Technol. 5139
[633] Oehmigen K, Haehnel M, Brandenburg R, Wilke C, Weltmann K D and von Woedtke T 2010 The role of acidification for antimicrobial activity of atmospheric pressure plasma in liquids Plasma Proc. Polym. 7 250-7

[634] Zhou W, Diwu Z, Panchuk-Voloshina N and Haugland R P 1997 A stable nonfluorescent derivative of resorufin for the fluorometric determination of trace hydrogen peroxide: applications in detecting the activity of phagocyte NADPH oxidase and other oxidases Anal. Biochem. 253 162-8

[635] Winter J, Wende K, Masur K, Iseni S, Duennbier M, Hammer M U, Tresp H, Weltmann K D and Reuter S 2013 Feed gas humidity: a vital parameter affecting a cold atmospheric-pressure plasma jet and plasma-treated human skin cells J. Phys. D: Appl. Phys. 46295401

[636] Yang F, Zhang R, He J and Abliz Z 2007 Development of a liquid chromatography electrospray ionization tandem mass spectrometric method for the determination of hydroxyl radical Rapid Commun. Mass Spectrom. 21 107-11

[637] Flyunt R, Leitzke A, Mark G, Mvula E, Reisz E, Schick R and von Sonntag C 2003 Determination of $\mathrm{OH}, \mathrm{O}_{2}^{-}$and hydroperoxide yields in ozone reactions in aqueous solution J. Phys. Chem. B 107 7242-53

[638] Staehelln J and Hoigne J 1985 Decomposition of ozone in water in the presence of organic solutes acting as promoters and inhibitors of radical chain reactions Environ. Sci. Technol. 19 1206-13

[639] Garner A L, St Corix C M, Pitt B R, Leikauf G D, Ando S and Koide K 2009 Specific fluorogenic probes for ozone in biological and atmospheric samples Nat. Chem. 1316-21

[640] Schweitzer C and Schmidt R 2003 Physical mechanisms of generation and deactivation of singlet oxygen Chem. Rev. 1031685

[641] Ragas X, Jimenez-Banzo A, Sanchez-Garcia D, Batllori X and Nonell S 2009 Singlet oxygen photosensitisation by the fluorescent probe singlet oxygen sensor green Chem. Commun. v 2920-2 